


\title{
Clinical Practice Guidelines for the Prevention and Management of Pain, Agitation/Sedation, Delirium, Immobility, and Sleep Disruption in Adult Patients in the ICU
}

\author{
John W. Devlin, PharmD, FCCM (Chair) ${ }^{1,2}$; Yoanna Skrobik, MD, FRCP(c), MSc, FCCM (Vice-Chair) ${ }^{3,4}$; \\ Céline Gélinas, RN, $\mathrm{PhD}^{5}$; Dale M. Needham, MD, $\mathrm{PhD}^{6}$; Arjen J. C. Slooter, $\mathrm{MD}, \mathrm{PhD}^{7}$; \\ Pratik P. Pandharipande, MD, MSCI, FCCM ${ }^{8}$; Paula L. Watson, MD'; Gerald L. Weinhouse, MD $^{10}$; \\ Mark E. Nunnally, MD, FCCM ${ }^{11,12,13,14}$; Bram Rochwerg, MD, MSc ${ }^{15,16}$; \\ Michele C. Balas, RN, PhD, FCCM, FAAN ${ }^{17}$; ; Mark van den Boogaard, RN, PhD ${ }^{19}$; Karen J. Bosma, MD ${ }^{20,21}$; \\ Nathaniel E. Brummel, MD, MSCI ${ }^{22,23}$; Gerald Chanques, MD, PhD ${ }^{24,25}$; Linda Denehy, PT, $\mathrm{PhD}^{26}$; \\ Xavier Drouot, MD, PhD ${ }^{27,28}$; Gilles L. Fraser, PharmD, MCCM ${ }^{29}$; Jocelyn E. Harris, OT, $\mathrm{PhD}^{30}$; \\ Aaron M. Joffe, DO, FCCM ${ }^{31}$; Michelle E. Kho, PT, PhD ${ }^{30}$; John P. Kress, MD ${ }^{32}$; Julie A. Lanphere, DO ${ }^{33}$; \\ Sharon McKinley, RN, PhD ${ }^{34}$; Karin J. Neufeld, MD, $\mathrm{MPH}^{35}$; Margaret A. Pisani, MD, $\mathrm{MPH}^{36}$; \\ Jean-Francois Payen, MD, PhD ${ }^{37}$; Brenda T. Pun, RN, DNP ${ }^{23}$; Kathleen A. Puntillo, RN, PhD, FCCM ${ }^{38}$; \\ Richard R. Riker, MD, FCCM ${ }^{29}$; Bryce R. H. Robinson, MD, MS, FACS, FCCM ${ }^{39}$; \\ Yahya Shehabi, MD, PhD, FCICM ${ }^{40}$; Paul M. Szumita, PharmD, FCCM ${ }^{41}$; \\ Chris Winkelman, RN, PhD, FCCM ${ }^{42}$; John E. Centofanti, MD, MSc ${ }^{43}$; Carrie Price, MLS ${ }^{44}$; \\ Sina Nikayin, $\mathrm{MD}^{45}$; Cheryl J. Misak, $\mathrm{PhD}^{46}$; Pamela D. Flood, $\mathrm{MD}^{47}$; Ken Kiedrowski, $\mathrm{MA}^{48}$; \\ Waleed Alhazzani, MD, MSc (Methodology Chair) ${ }^{16,49}$
}

${ }^{1}$ School of Pharmacy, Northeastern University, Boston, MA.

${ }^{2}$ Division of Pulmonary, Critical Care and Sleep Medicine, Tufts Medical Center, Boston, MA.

${ }^{3}$ Faculty of Medicine, McGill University, Montreal, QC, Canada.

${ }^{4}$ Regroupement de Soins Critiques Respiratoires, Réseau de Santé Respiratoire, Montreal, QC, Canada.

${ }^{5}$ Ingram School of Nursing, McGill University, Montreal, QC, Canada.

${ }^{6}$ Division of Pulmonary and Critical Care Medicine, Department of Physical Medicine and Rehabilitation, School of Medicine, Johns Hopkins University, Baltimore, MD.

${ }^{7}$ Department of Intensive Care Medicine, Brain Center Rudolf Magnus, University Medical Center, Utrecht University, Utrecht, The Netherlands.

${ }^{8}$ Department of Anesthesiology, Division of Anesthesiology Critical Care Medicine, Vanderbilt University Medical Center, Nashville, TN.

${ }^{9}$ Division of Sleep Medicine, Vanderbilt University Medical Center, Nashville, TN.

${ }^{10}$ Division of Pulmonary and Critical Care, Brigham and Women's Hospital and School of Medicine, Harvard University, Boston, MA.

${ }^{11}$ Division of Anesthesiology, Perioperative Care and Pain Medicine, New York University Langone Health, New York, NY.
${ }^{12}$ Division of Medicine, New York University Langone Health, New York, NY.

${ }^{13}$ Division of Neurology, New York University Langone Health, New York, NY.

${ }^{14}$ Division of Surgery, New York University Langone Health, New York, NY. ${ }^{15}$ Department of Medicine (Critical Care), McMaster University, Hamilton, ON, Canada.

${ }^{16}$ Department of Health Research Methods, Impact and Evidence, McMaster University, Hamilton, ON, Canada.

${ }^{17}$ The Ohio State University, College of Nursing, Center of Excellence in Critical and Complex Care, Columbus, $\mathrm{OH}$.

${ }^{18}$ The Ohio State University Wexner Medical Center, Columbus, $\mathrm{OH}$.

${ }^{19}$ Department of Intensive Care Medicine, Radboud University Medical Center, Nijmegen, The Netherlands.

${ }^{20}$ Division of Critical Care, London Health Sciences Centre, London, ON, Canada.

${ }^{21}$ Schulich School of Medicine \& Dentistry, University of Western Ontario, London, ON, Canada.

${ }^{22}$ Center for Quality Aging, Division of Allergy, Pulmonary and Critical Care Medicine, Department of Medicine, Vanderbilt University Medical Center, Nashville, TN.

${ }^{23}$ Center for Health Services Research, Vanderbilt University Medical Center, Nashville, TN. 
${ }^{24}$ Department of Anesthesia and Intensive Care, Montpellier University Saint Eloi Hospital, Montpellier, France.

${ }^{25}$ PhyMedExp, INSERM, CNRS, University of Montpellier, Montpellier, France.

${ }^{26}$ Melbourne School of Health Sciences, University of Melbourne, Melbourne, VIC, Australia.

${ }^{27}$ Faculte de Medecine Pharmacie, University of Poitiers, Poitiers, France.

${ }^{28}$ Service de Neurophysiologie, $\mathrm{CHU}$ de Poitiers, Poitiers, France.

${ }^{29}$ Department of Critical Care, Maine Medical Center and School of Medicine, Tufts University, Portland, ME.

${ }^{30}$ School of Rehabilitation Science, McMaster University, Hamilton, ON, Canada.

${ }^{31}$ Department of Anesthesiology and Pain Medicine, Harborview Medical Center, University of Washington, Seattle, WA.

${ }^{32}$ Division of Pulmonary and Critical Care Medicine, University of Chicago, Chicago, IL.

${ }^{33}$ Department of Physical Medicine and Rehabilitation, Intermountain Healthcare, Salt Lake City, UT.

${ }^{34}$ School of Nursing and Midwifery, Deakin University, Geelong, VIC, Australia.

${ }^{35}$ Department of Psychiatry and Behavioral Sciences, Johns Hopkins University School of Medicine, Baltimore, MD.

${ }^{36}$ Division of Pulmonary, Critical Care and Sleep Medicine, School of Medicine, Yale University, New Haven, CT.

${ }^{37}$ Department of Anesthesiology and Critical Care, Grenoble Alpes University Hospital, Grenoble, France.

${ }^{38}$ School of Nursing, University of California San Francisco, San Francisco, CA.

${ }^{39}$ Department of Surgery, University of Washington, Seattle, WA.

${ }^{40}$ Department of Critical Care and Perioperative Medicine, School of Clinical Sciences, Monash University, Melbourne, VIC, Australia.

${ }^{41}$ Department of Pharmacy, Brigham and Women's Hospital, Boston, MA.

${ }^{42}$ Frances Payne Bolton School of Nursing, Case Western Reserve University, Cleveland, $\mathrm{OH}$.

${ }^{43}$ Department of Anesthesia and Critical Care, McMaster University, Hamilton, ON, Canada.

${ }^{44}$ Welch Medical Library, Johns Hopkins University, Baltimore, MD.

${ }^{45}$ Department of Psychiatry and Behavioral Sciences, New York Medical College, Valhalla, NY.

${ }^{46}$ Department of Philosophy, University of Toronto, Toronto, CA.

${ }^{47}$ Division of Anesthesiology, Stanford University Hospital, Palo Alto, CA.

${ }^{48}$ Patient and Family Advisory Committee, Johns Hopkins Hospital, Baltimore, MD.

${ }^{49}$ Department of Medicine (Critical Care and Gastroenterology), McMaster University, Hamilton, ON, Canada.

These guidelines are endorsed by the American Association of CriticalCare Nurses, American College of Chest Physicians, American College of Clinical Pharmacy, American Delirium Society, Australian College of Critical Care Nurses, Canadian Critical Care Society, Eastern Association for the Surgery of Trauma, European Delirium Association, European Federation of Critical Care Nursing Associations, Neurocritical Care Society, and Society of Critical Care Anesthesiologists.

Supplemental digital content is available for this article. Direct URL citations appear in the printed text and are provided in the HTML and PDF versions of this article on the journal's website (http://journals.lww.com/ccmjournal).

Dr. Devlin has received research funding from the National Institute of Aging, National Heart, Lung and Blood Institute, and AstraZeneca Pharmaceuticals, he is on the editorial board of Critical Care Medicine, and he is the president of the American Delirium Society. Dr. Skrobik participates in the ATS and the American College of Chest Physicians (ACCP), and she is on the editorial board for Intensive Care Medicine and Chest. Dr. Needham is a principal investigator on a National Institutes of Health (NIH)-funded, multicentered randomized trial (R01HL132887) evaluating nutrition and exercise in acute respiratory failure and, related to this trial, is currently in receipt of an unrestricted research grant and donated amino acid product from Baxter Healthcare and an equipment loan from Reck Medical Devices to two of the participating study sites, external to his institution. Dr. Slooter has disclosed that he is involved in the development of an electroencephalogram-based delirium monitor, where any (future) profits from electroencephalogram-based delirium monitoring will be used for future scientific research only. Dr. Pandharipande's institution received funding from Hospira (research grant to purchase study drug [dexmedetomidine] in collaboration with a NIH-funded RO1 study) and disclosed that he is the past president of the American Delirium Society. Dr. Nunnally participates in the Society of Critical Care Anesthesiologists, International Anesthesia Research Society, and American Society of Anesthesiology (ASA). Dr. Rochwerg participates as a guideline methodologist for other organizations (i.e., American Thoracic Society [ATS] and Canadian Blood Service) in addition to the Society of Critical Care Medicine. Dr. Balas received funding from Select Medical (primary investigator on research study exploring Assess, Prevent, and Manage Pain, Both Spontaneous Awakening Trials and Spontaneous Breathing Trials, Choice of analgesia and sedation, Delirium: Assess, Prevent, and Manage, Early mobility and Exercise, and Family engagement and empowerment bundle adoption). Dr. Bosma received funding from the Canadian Institutes of Health Research (ClHR) where she is the primary investigator of an industry partnered research grant with Covidien as the industry partner of the CIHR for a study investigating proportional assist ventilation versus pressure support ventilation for weaning from mechanical ventilation. Dr. Brummel participates in the ATS (Aging and Geriatrics Working Group CoChair) and ArjoHuntleigh (advisory board activities). Dr. Chanques participates in other healthcare professional organization activities. Dr. Denehy participates in the Australian Physiotherapy Association. Dr. Drouot participates in the French Sleep Society and the French Institute for Sleep and Vigilance. Mr. Joffe participates on committees for ASA. Dr. Kho received funding from Restorative Therapies (Baltimore, MD) (loaned two supine cycle ergometers for ongoing research). Dr. Kress received funding from a dexmedetomidine speaker program, he participates in the ATS and ACCP, and he has served as an expert witness in medical malpractice. Dr. McKinley participates in the American Association of Critical-Care Nurses (AACN) (editorial board of American Journal of Critical Care) and the American Heart Association (editorial board of Journal of Cardiovascular Nursing). Dr. Neufeld participates in the American Delirium Society (board member). Dr. Pisani participates in the ACCP (Chair Scientific Programming Committee and Steering Committee Women's Health Network). Dr. Payen received funding from Baxter SA (distributor of dexmedetomidine in France), and he has received honorariums from Baxter SA (oral presentations of dexmedetomidine). Ms. Pun participates as an AACN speaker at the National Conference. Dr. Puntillo participates in other healthcare professional organizations (e.g., AACN). Dr. Robinson participates in the Easter Association for the Surgery of Trauma, American College of Surgeons, and American Association for the Surgery of Trauma. Dr. Shehabi received funding from an unrestricted research grant (drug supply) from Pfizer (Hospira) and Orion Pharma to an ongoing multinational multicenter study. Mr. Szumita participates in several committees for the American Society of Health-System Pharmacists. Ms. Price has disclosed that she is a medical librarian working at Johns Hopkins University, and she consults as an information specialist to the Cochrane Urology Review Group. Dr. Flood participates on the Society of Obstetric Anesthesia and Perinatology research committee and the ASA Chronic Pain Committee. The remaining authors have disclosed that they do not have any potential conflicts of interest.

The American College of Critical Care Medicine (ACCM), which honors individuals for their achievements and contributions to multidisciplinary critical care medicine, is the consultative body of the Society of Critical Care Medicine (SCCM) that possesses recognized expertise in the practice of critical care. The College has developed administrative guidelines and clinical practice parameters for the critical care practitioner. New guidelines and practice parameters are continually developed, and current ones are systematically reviewed and revised.

For information regarding this article, E-mail: j.devlin@neu.edu

Objective: To update and expand the 2013 Clinical Practice Guidelines for the Management of Pain, Agitation, and Delirium in Adult Patients in the ICU.

Design: Thirty-two international experts, four methodologists, and four critical illness survivors met virtually at least monthly. All section groups gathered face-to-face at annual Society of Critical Care Medicine congresses; virtual connections included those unable to attend. A formal conflict of interest policy was developed a priori and enforced throughout the process. Teleconferences and 
electronic discussions among subgroups and whole panel were part of the guidelines' development. A general content review was completed face-to-face by all panel members in January 2017.

Methods: Content experts, methodologists, and ICU survivors were represented in each of the five sections of the guidelines: Pain, Agitation/sedation, Delirium, Immobility (mobilization/rehabilitation), and Sleep (disruption). Each section created Population, Intervention, Comparison, and Outcome, and nonactionable, descriptive questions based on perceived clinical relevance. The guideline group then voted their ranking, and patients prioritized their importance. For each Population, Intervention, Comparison, and Outcome question, sections searched the best available evidence, determined its quality, and formulated recommendations as "strong," "conditional," or "good" practice statements based on Grading of Recommendations Assessment, Development and Evaluation principles. In addition, evidence gaps and clinical caveats were explicitly identified.

Results: The Pain, Agitation/Sedation, Delirium, Immobility (mobilization/rehabilitation), and Sleep (disruption) panel issued 37 recommendations (three strong and 34 conditional), two good practice statements, and 32 ungraded, nonactionable statements. Three questions from the patient-centered prioritized question list remained without recommendation.

Conclusions: We found substantial agreement among a large, interdisciplinary cohort of international experts regarding evidence supporting recommendations, and the remaining literature gaps in the assessment, prevention, and treatment of Pain, Agitation/sedation, Delirium, Immobility (mobilization/rehabilitation), and Sleep (disruption) in critically ill adults. Highlighting this evidence and the research needs will improve Pain, Agitation/sedation, Delirium, Immobility (mobilization/rehabilitation), and Sleep (disruption) management and provide the foundation for improved outcomes and science in this vulnerable population. (Crit Care Med 2018; 46:e825-e873)

Key Words: delirium; guidelines; immobility; intensive care; mobilization; pain; sedation; sleep

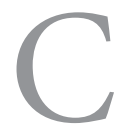

linical practice guidelines are published, often by professional societies, because they provide a current and transparently analyzed review of relevant research with the aim to guide clinical practice. The 2018 Pain, Agitation/ sedation, Delirium, Immobility (rehabilitation/mobilization), and Sleep (disruption) (PADIS) guideline builds on this mission by updating the 2013 Pain, Agitation, and Delirium (PAD) guidelines (1); by adding two inextricably related clinical care topics-rehabilitation/mobilization and sleep; by including patients as collaborators and coauthors; and by inviting an international panel of experts from high-income countries as an early step toward incorporating more diverse practices and expertise from the global critical care community.

Readers will find rationales for 37 recommendations (derived from actionable Patient, Intervention, Comparison, and Outcome [PICO] questions); two ungraded good practice statements (derived from actionable PICO questions where it is unequivo$\mathrm{cal}$, the benefits of the intervention outweigh the risks but direct evidence to support the intervention does not exist); and 32 ungraded statements (derived from nonactionable, descriptive questions) across the five guideline sections. The supplemental digital content figures and tables linked to this guideline provide background on how the questions were established, profiles of the evidence, the evidence-to-decision tables used to develop recommendations, and voting results. Evidence gaps and future research directions are highlighted in each section. The five sections of this guideline are interrelated, and thus, the guideline should be considered in its entirety rather than as discrete recommendations.

Knowledge translation and implementation effectiveness are an important segue to our guideline document and work to foster advances in clinical practice related to PADIS assessment, prevention, and treatment. A PADIS guideline implementation and integration article separately created to facilitate this is available (2). Many challenges characterize developing effective PADIS-related educational and quality improvement programs. Although some have not achieved expected outcomes $(3,4)$, many quality improvement efforts in this field have been successful (5-10).

\section{METHODS}

The panel followed the Grading of Recommendations Assessment, Development and Evaluation (GRADE) working group's methodology for clinical practice guideline development. Guideline chairs, with input from the methodology team, created a protocol before beginning formal work on the guideline. Chairs, group heads, and panel members, with input from ICU survivors (11), selected topics that are important to patients and practicing clinicians. A list of questions was developed for each topic, and questions and outcomes were prioritized through an electronic survey following the GRADE principles (12).

Once the list of questions was finalized, a university-based librarian conducted a literature review of five electronic databases from 1990 to October 2015 based on priority topics voted on by the members and revised by critical illness survivors. The librarian finalized the relevant search terms with the groups and extracted literature based on these prioritized topics. These publications were then evaluated for their methodologic rigor that determined the highest quality of evidence available per outcome and per question in keeping with GRADE guidance. Evidence evaluation was performed by determining its relevance for each question; members with a financial or intellectual conflict of interest did not review questions related to their conflict. Full-text screening was performed in duplicate. Each group used the GRADE evidenceto-decision framework to formulate the preliminary recommendations (12). Further, all five groups' comments on the overall recommendations and the literature provided to support it were reviewed by the chair and vice-chair after recommendation voting and screened for potential or perceived conflict.

Subsequently, recommendations were discussed in person among the full panel. Then, only members who were free of overt or potential conflict of interest voted electronically for each recommendation. We defined consensus as greater than $80 \%$ agreement with greater than $70 \%$ response rate. ICU survivors participated in every step of the guideline development, which provided a unique perspective for this guideline. We used the GRADE criteria to formulate good practice statements where appropriate (11). For nonactionable, descriptive questions, evidence was 
summarized and ungraded statements were provided. A complete description of the methods is found in Supplemental Appendix 1 (Supplemental Digital Content 1, http://links.lww.com/CCM/ D759). A detailed description of the methodologic innovations that characterize these guidelines is published separately (13).

\section{PAIN}

Pain management is complex because pain patterns are highly individual (e.g., acute, chronic, and acute-on-chronic), it arises from different sources (e.g., somatic, visceral, and neuropathic), and patients have subjective perceptions and have exceedingly variable tolerability. A consistent approach to pain assessment and management is paramount given the unique features of critically ill adults that include impaired communication, altered mental status, mechanical ventilation, procedures and use of invasive devices, sleep disruption, and immobility/mobility status (14).

Critically ill adults experience moderate-to-severe pain at rest (15) and during standard care procedures (16). Pain is defined as "an unpleasant sensory and emotional experience associated with actual or potential tissue damage, or described in terms of such damage" (17). Pain should be considered to be "whatever" the experiencing person says it is, existing "whenever" the experiencing person says it does (18). Although the reference standard measure of pain is a patient's self-report, the inability to communicate clearly does not negate a patient's pain experience or the need for appropriate pain management (19). Fortunately, validated behavioral pain scales provide alternative measures for pain assessment in those patients unable to self-report their pain. Severe pain negatively affects patient status (e.g., cardiac instability, respiratory compromise, immunosuppression) in critically ill adults; implementation of assessment-driven and standardized pain management protocols improves ICU outcomes and clinical practice $(5,20)$. Carefully titrated analgesic dosing is important when balancing the benefits versus potential risks of opioid exposure (21-25). In this guideline section, we address three actionable questions and two descriptive questions related to the pain experience of critically ill adults (see prioritized topic list in Supplemental Table 1 [Supplemental Digital 2, http://links.lww.com/CCM/D760] and voting results in Supplemental Table 2 [Supplemental Digital Content 3, http://links.lww.com/CCM/D761]). The evidence summaries and evidence-to-decision tables used to develop recommendations for the pain group are available in Supplemental Table 3 (Supplemental Digital Content 4, http:// links.lww.com/CCM/D762), and the forest plots for all metaanalyses are available in Supplemental Figure 1 (Supplemental Digital Content 5, http://links.lww.com/CCM/D763).

\section{Risk Factors}

Question: What factors influence pain in critically ill adults during both rest and during procedures?

Ungraded Statements: Pain at rest is influenced by both psychologic (e.g., anxiety and depression) and demographic (e.g., young age, one or more comorbidities, and history of surgery) factors.

Pain during a procedure is influenced by preprocedural pain intensity, the type of procedure, underlying surgical or trauma diagnoses, and demographic factors (younger age, female sex, and non-white ethnicity).

Rationale: Pain is common in critically ill adults at rest and during procedures including regular activities (e.g., turning) and discrete procedures (e.g., arterial catheter insertion). The prior guidelines document the incidence, frequency, severity, and impact of pain (1): 1) adult medical, surgical, and trauma ICU patients routinely experience pain, both at rest and during standard ICU care; 2) procedural pain is common in adult ICU patients; and 3) pain in adult cardiac surgery patients is common and poorly treated; women experience more pain than men. This guideline's new descriptive question focuses on observational studies that have identified factors associated with pain in ICU patients at rest and during procedures.

During Rest. Five studies (evaluating from 74 to 5,176 patients each) describe factors associated with pain in medical, surgical, and trauma ICU populations (26-30). The time from pain recognition to analgesic initiation, the pain being worse than what the patient expected, and ICU length of stay (LOS) are significant predictors of higher self-reported pain intensity (26). The amount of analgesic administered after cardiac and abdominal surgery in the ICU is a significant predictor of later pain intensity, pain affect (i.e., emotional experience), and pain sensation (i.e., quality of pain related to the sensory dimension of the pain experience) (27). Among 301 mechanically ventilated patients, younger age and prior surgery both predicted greater pain at rest (28). After cardiac surgery, patients with preoperative anxiety or depression have a higher level of selfreported pain intensity (29). One large cohort of 5,176 medical ICU adults reported the following baseline predictors of higher self-reported pain intensity during the ICU admission: younger age; need for support to conduct daily living activities; number of comorbidities such as cardiac and pulmonary diseases; depression; anxiety; and an expectation of a future poor quality of life (30). Clinicians should make an effort to obtain information from all relevant sources, including family and other caregivers, about their patient's pre-ICU illness background to better consider these factors in plans to improve patient comfort.

During Procedures. A total of 12 studies (evaluating from 30 to 5,957 patients each) have evaluated pain level, mostly through patient self-reports, during 12 different procedures in various ICU populations (i.e., medical, surgical, cardiovascular, trauma, and neurologic) $(27,28,31-37)$. The following procedures are associated with the greatest increased pain intensity: arterial catheter insertion, chest tube removal (CTR), wound drain removal (16), turning (32) and repositioning, and tracheal suctioning (37). (A complete list of painful procedures can be found in Supplemental Table 4 [Supplemental Digital Content 6, http://links.lww.com/CCM/D764].) Patients with a surgical history/diagnosis or trauma had worse procedural pain (32), as did younger (37), female (33), and non-white patients $(34,37)$; however, in one report evaluating six procedures (35), no association was found between procedural pain intensity and age except during wound care and tracheal suctioning.

Opioid use before or during a procedure was found to be a risk factor for higher procedural pain in one recent, large multinational 
study (16), but not in a smaller, older study limited to surgical ICU patients (27). This divergence may be due to a focus on the dose rather than efficacy of opioid therapy, mistimed opioid administrations (relative to the procedure), and the inclusion of patients with prior opioid exposure. Such findings emphasize the importance of preprocedural pain assessment and preemptive analgesia, when appropriate, for procedures known to cause pain. Indeed, severe procedural pain is associated with severe adverse events (e.g., tachycardia, bradycardia, hypertension, hypotension, desaturation, bradypnea, and ventilator distress) (21) that may be prevented with appropriate pain assessment and preemptive analgesia.

Evidence Gaps: Future research should include the following: 1) an exploration of the affect of sociodemographic variables such as age, gender, and ethnicity that may affect pain and response to pharmacologic intervention; 2) identification of pharmacokinetic, pharmacogenomic, and gender-associated factors that influence analgesic responses; 3 ) a determination of what painrelated behaviors predict self-reported pain; 4) the development and study of objective measures (e.g., pupillary reflex dilatation response) to determine pain before and during a planned procedure in patients unable to self-report pain; 5) identification of biomarkers associated with pain; 6) conduct of clinical trials of pain management interventions during procedures; and 7) investigation of the relationship among opioid effectiveness, opioid tolerance, opioid-related hyperalgesia, and procedural pain (38).

\section{Assessment}

Question: What are the most reliable and valid pain assessment methods to use in critically ill adults?

\section{Self-Report Scales.}

Ungraded Statements: A patient's self-report of pain is the reference standard for pain assessment in patients who can communicate reliably.

Among critically ill adults who are able to self-report pain, the $0-10$ Numeric Rating Scale (NRS) administered either verbally or visually is a valid and feasible pain scale.

Rationale: Four studies served to answer the above question (39-42). One study evaluated 111 medical/surgical ICU patients for pain in a randomized order using five different self-report scales: 1) 0-10 cm Visual Analog Scale Horizontal (VAS-H); 2) 0-10 cm Visual Analog Scale (VAS) Vertical; 3) Verbal Descriptor Scale (VDS): no pain, mild pain, moderate pain, severe pain, and extreme pain); 4) 0-10 NRS Oral (NRS-O); and 5) 0-10 NRS Visual (NRS-V) in a horizontal format (39). The NRS-V had the highest rate of success (i.e., response obtained) (91\%); the VAS-H the lowest (66\%). The NRS-V success rate was significantly greater than the VDS and VAS (both $p<0.001)$ and NRS-O $(p<0.05)$. It also had the best sensitivity, negative predictive value, and accuracy; given its ease of use, it was most highly favored by ICU patients.

The 0-10 Faces Pain Thermometer (FPT) $(4.25 \times 14$ vertical format) scale, validated in 105 postoperative cardiac surgery ICU patients, revealed higher FPT scores during turning and good correlation with the VDS for pain supporting its construct validity (43). Patients evaluated the faces and numbers in the FPT favorably and nearly all rated it as easy to use and useful in identifying pain intensity. When compared with the 0-10 NRS, the Wong-Baker FACES scale resulted in higher pain scores suggesting that pain scales developed for children should be evaluated cautiously before being used in adults (41). Finally, in another study (42), cardiovascular surgery ICU patients stated that the 0-10 NRS or Verbal Rating Scale (VRS) of six descriptors scale is better for evaluating their pain than the 0-100 VAS; they prefer to have their pain evaluated with the VRS (vs the 0-10 NRS). In summary, the $0-10$ NRS in a visual format is the best self-reported pain scale to use in critically ill adults. A descriptive pain scale like the VDS should be considered for ICU patients unable to use a numerically formatted scale such as the $0-10$ NRS.

\section{Behavioral Assessment Tools.}

Ungraded Statement: Among critically ill adults unable to self-report pain and in whom behaviors are observable, the Behavioral Pain Scale in intubated (BPS) and nonintubated (BPS-NI) patients and the Critical-Care Pain Observation Tool (CPOT) demonstrate the greatest validity and reliability for monitoring pain.

Rationale: We updated this psychometric analysis of behavioral pain assessment tools, which was initiated in the 2013 guidelines (1) and in a systematic review (44). Fifty-three articles pertained to the development, validation, and implementation of 12 pain scales for use in critically ill adults unable to self-report pain. Four additional pain scales were included: the FACES Scale (45), the Facial Action Coding System (46), the Pain In Advanced Dementia (PAINAD) (47), and the Behavior Pain Assessment Tool (BPAT) (48). In this analysis, we considered a pain scale with a psychometric quality score of 15-20 to have very good psychometric properties; a score of 12-14.9 good psychometric properties; 10-11.9 some acceptable psychometric properties; and 0-9.9 very few psychometric properties reported and/or unacceptable results $(1,49)$. A list of studies (by pain scale) published since 2013 are included in Supplemental Table 5 (Supplemental Digital Content 7, http://links.lww.com/CCM/D765), and the psychometric scores and the quality of evidence supporting each pain scale are described in Supplemental Table 6 (Supplemental Digital Content 8, http://links.lww.com/CCM/D766).

The CPOT and the BPS remain the most robust scales for assessing pain in critically ill adults unable to self-report. Each has very good psychometric properties with scores of 16.7 and 15.1, respectively. The BPS-NI obtained a psychometric weighted score of 14.8. Although both the BPS and the CPOT have been validated across large samples of medical, surgical, and trauma ICUs (50-54), studies involving brain-injured patients using the BPS $(50,51)$ and CPOT $(52-54)$ are small. In the brain-injured population, although the construct validity of both scales is supported with higher scores during painful procedures (vs rest and nonpainful procedures), patients predominantly expressed pain-related behaviors that were related to level of consciousness; grimacing and muscle rigidity were less frequently observed (50, 52-54). An additional study (51), although not evaluating validity, found that BPS and BPS-NI were feasible and reliable to use in the brain-injured population. 
Of note, Behavioral Pain Scales have been validated in the following languages (other than French or English): CPOTMandarin (55), Korean (56), Spanish (57), and Swedish (58); BPS and BPS-NI-Mandarin (59).

The BPAT, the first behavioral pain assessment tool to undergo international validation, obtained a psychometric weighted score of 10.6 when tested in its original English version and 12 other languages among 3,851 critically ill adults from 28 countries (48). This is less than reported for either the BPS or the CPOT because the feasibility and impact of its use once implemented in clinical practice remain to be investigated. By the time this implementation research is complete, it may be of use in countries/languages where neither the BPS nor CPOT has been validated (48). Each of the other scales considered (i.e., the Face, Legs, Activity, Cry, Consolability; the Non-verbal Pain Assessment Tool; the PAIN; the BOT; the FACES; the Fear-Avoidance Components Scale; and the PAINAD) had low psychometric weighted scores $(<10)$.

\section{Proxy Reporters.}

Ungraded Statement: When appropriate, and when the patient is unable to self-report, family can be involved in their loved one's pain assessment process.

Rationale: The intensity and distress of 10 different patient symptoms, including pain, were independently assessed by ICU patients, nurses, physicians, and family members (60). For both pain intensity and pain distress, the reports of family proxy reporters were found to be closer to ICU patients' self-reports than that of the patients' nurses and physicians. However, the agreement between family and patients was only moderate. A second study compared ICU nurse and patient pain perception across nine procedures using a 10-point scale. Although patient and nurse pain scores for nasogastric tube insertion and tracheal aspiration were similar, they were significantly higher among nurses (vs patients) for position change, subcutaneous injection, blood sugar testing, and blood pressure (BP) measurement (61). No statistical measure of agreement between nurse and ICU patient scores was reported. Finally, compared with seriously ill patients' self-reports, surrogates correctly identified pain presence $74 \%$ of the time and pain severity $53 \%$ of the time, with a tendency to overestimate pain intensity (62). There are families who may not want to be involved in pain assessment or situations where family involvement in pain assessment is not appropriate. Family involvement in pain assessment should not substitute for an ICU team's role and commitment to systematic pain assessment and optimal analgesia.

\section{Physiologic Measures.}

Ungraded Statement: Vital signs (VS) (i.e., heart rate [HR], $\mathrm{BP}$, respiratory rate $[\mathrm{RR}]$, oxygen saturation $\left[\mathrm{SpO}_{2}\right]$, and endtidal $\mathrm{CO}_{2}$ ) are not valid indicators for pain in critically ill adults and should only be used as cues to initiate further assessment using appropriate and validated methods such as the patient's self-report of pain (whenever possible) or a behavioral scale (i.e., BPS, BPS-NI, CPOT).

Rationale: The 2013 guidelines state that VS should not be used alone to assess pain in critically ill adults (1). Fourteen studies (four new since the 2013 guidelines) ( $n=30-755$ patients) evaluated the validity of using VS for pain assessment across various ICU populations and reported inconsistent results $(31,34,37,63-73)$. In 11 of 14 studies, HR and/ or BP was found to increase when ICU patients were exposed to a nociceptive procedure (e.g., endotracheal/tracheal suctioning) compared with either rest or a nonnociceptive procedure (e.g., cuff inflation, eye care) $(34,37,63-71)$. However, these HR and BP increases ( $<20 \%$ in all studies) were not considered to be clinically significant by the authors. In addition, VS were found to increase during both nociceptive and nonnociceptive procedures suggesting the lack of validity of these indicators $(68,70,72-74)$. In some studies, RR increased and/or end-tidal $\mathrm{CO}_{2}$ decreased during a painful procedure $(64,65,68)$, whereas $\mathrm{SpO}_{2}$ decreased $(65,69)$. Except for associations found among these VSs (i.e., $\mathrm{HR}, \mathrm{RR}$, and $\mathrm{SpO}_{2}$ ) and the pain described by cardiac surgery ICU patients themselves (64) and by critically ill adults with a traumatic brain injury (TBI) (74), an association between VS changes and patients' self-reported pain was not observed $(65,67,68,70)$. In one quality improvement project (19), changes in VS (e.g., tachycardia, bradycardia, hypertension, hypotension, desaturation, and bradypnea) during nursing care (bathing, massage, sheet-change, repositioning) were considered as severe pain-related adverse events. Although VS changes can be considered to be pain-related adverse events, they should not be used for pain assessment in critically ill adults.

Evidence Gaps: When evaluating self-reported pain intensity scales, further research comparing FACES pain scales with other rating scales (e.g., NRS, VDS, and VAS) in heterogeneous ICU populations is required. Family members' acting as proxy reporters using behavioral pain assessment tools (e.g., BPS/ BPS-NI and CPOT) for ICU patients unable to self-report should be explored. Behavioral scales are the alternative measures to use when the patient is unable to self-report (75). Scale revisions could enhance the validity of their use in ICU patients with brain injury and other neurologically critically ill patients (such as those with neuromuscular diseases); research on the application of the BPAT in ICU practice is encouraged. However, situations exist for which behavioral scales are impossible to use (e.g., unresponsive patients with a Richmond Agitation-Sedation Scale [RASS] $\leq-4$ ). In such situations, no alternative methods are currently available to ICU clinicians. Other technology that may be useful in the ICU pain assessment process should be explored. Technology measuring HR variability (e.g., the Analgesia Nociception Index) $(76,77)$ or incorporating simultaneously different physiologic parameters (e.g., Nociception Level Index) (78) may be relevant. Pupillary reflex dilation using video pupillometry has shown promising results in pain assessment of critically ill adults (79-81), but future research is necessary to investigate the benefits, harms, and feasibility of implementation in the ICU.

\section{Pharmacologic Adjuvants to Opioid Therapy}

Opioids remain a mainstay for pain management in most ICU settings. However, their side effects preoccupy clinicians because of 
important safety concerns, such as sedation, delirium, respiratory depression, ileus, and immunosuppression, may lengthen ICU LOS and worsen post-ICU patient outcome. A "multi-modal analgesia" approach has been used in the perioperative setting to reduce opioid use and to optimize postoperative analgesia and rehabilitation (82). Nonopioid analgesics such as acetaminophen, nefopam, ketamine, lidocaine, neuropathic agents, and nonsteroidal anti-inflammatory drugs (NSAIDs) have each been evaluated in critically ill adults with the aim of sparing opioid use and improving analgesic effectiveness. In addition to opioids, these nonopioid analgesic alternatives may be combined with regional anesthetics and nonpharmacologic interventions known to reduce pain (see below). Dose, duration, and pharmacologic effectiveness need to be evaluated when combination strategies are being evaluated.

\section{Acetaminophen.}

Question: Should acetaminophen be used as an adjunct to an opioid (vs an opioid alone) for pain management in critically ill adults?

Recommendation: We suggest using acetaminophen as an adjunct to an opioid to decrease pain intensity and opioid consumption for pain management in critically ill adults (conditional recommendation, very low quality of evidence).

Rationale: Two single-centered, parallel-group randomized controlled trials (RCTs) evaluated IV acetaminophen $1 \mathrm{~g}$ every 6 hours (q6h) versus placebo in a double-blind fashion in 113 postcardiac surgery patients (83) and in an open design in 40 postabdominal surgical ICU patients (84). After 24 hours, pooled analysis of these two trials revealed a decrease in pain intensity at rest measured by the VAS-H (mean difference [MD], -0.5 points; $95 \% \mathrm{CI},-0.7$ to -0.2 ; moderate quality) and in opioid consumption (MD, $-4.5 \mathrm{mg}$ [morphine equivalents]; $95 \% \mathrm{CI},-6.6$ to -2.5 ; moderate quality) in the acetaminophen groups. In the study demonstrating the greatest reduction in opioid consumption (84), time to extubation, sedation, and nausea rate were all significantly improved in the acetaminophen group. The risk for IV acetaminophen-associated hypotension (a decrease in the mean arterial pressure $>15 \mathrm{~mm} \mathrm{Hg}$ may occur in up to $50 \%$ of patients) may preclude its use in some patients (85). Given these findings, panel members suggest using acetaminophen (IV, oral, or rectal) to decrease pain intensity and opioid consumption when treating pain in critically ill patients, particularly in patients at higher risk for opioid-associated safety concerns (e.g., critically ill patient recovering from abdominal surgery and at risk for ileus or nausea and vomiting). Although IV acetaminophen was the intervention evaluated in the two relevant studies, the panel felt that this conditional recommendation was generalizable to all acetaminophen administration routes. Although not studied in the critically ill, the absorption (i.e., bioavailability) of acetaminophen administered by the oral or rectal route may be reduced in some critically ill subgroups (e.g., those requiring vasopressor support). The IV route of administration may be preferable in these situations, balanced with the hypotension risk described with IV (but not enteral) acetaminophen administration. The acquisition cost and availability of IV acetaminophen vary widely among countries and will likely influence the decision to use this specific formulation of acetaminophen in critically ill adults.

\section{Nefopam.}

Question: Should nefopam be used either as an adjunct or a replacement for an opioid (vs an opioid alone) for pain management in critically ill adults?

Recommendation: We suggest using nefopam (if feasible) either as an adjunct or replacement for an opioid to reduce opioid use and their safety concerns for pain management in critically ill adults (conditional recommendation, very low quality of evidence).

Rationale: Nefopam is a nonopioid analgesic that exerts its effect by inhibiting dopamine, noradrenaline, and serotonin recapture in both the spinal and supraspinal spaces. A 20-mg dose has an analgesic effect comparable to $6 \mathrm{mg}$ of IV morphine (86). Unlike non-cyclooxygenase (COX)-1 selective NSAIDs (e.g., ketorolac), nefopam has no detrimental effects on hemostasis, the gastric mucosa, or renal function; unlike acetaminophen, it has no detrimental effects on hepatic function, and unlike opioids, it has no detrimental effects on vigilance, ventilatory drive, and intestinal motility. However, nefopam use can be associated with tachycardia, glaucoma, seizure, and delirium. Nevertheless, nefopam may be a safe and effective alternative or adjunctive analgesic for ICU patients. Although not available in United States and Canada, nefopam is a low-cost drug that is used in nearly 30 countries. For example, after acetaminophen, it is the second most frequently used nonopioid medication in mechanically ventilated ICU patients in France (87).

A three-armed, double-blind, noninferiority RCT tested the effect of nefopam, fentanyl, and combination nefopam + half-dose fentanyl, administered by a patient-controlled analgesia (PCA) device, in 276 elective cardiac surgery patients in one ICU (88). Patients' self-reported pain intensity was not significantly different among the three groups despite similar PCA volumes. Nausea was significantly more frequent in the fentanyl group compared with nefopam groups. If available, nefopam could be used to reduce the opioid consumption and opioid-associated side effects, such as nausea, after an evaluation of the risk-to-benefit ratio of all available analgesic options and patient reassessment for potential side effects (tachycardia, glaucoma, seizure, and delirium) (89-92).

\section{Ketamine.}

Question: Should ketamine be used as an adjunct to an opioid (vs an opioid alone) for pain management in critically ill adults?

Recommendation: We suggest using low-dose ketamine $(0.5 \mathrm{mg} / \mathrm{kg}$ IVP $\mathrm{x} 1$ followed by $1-2 \mu \mathrm{g} / \mathrm{kg} / \mathrm{min}$ infusion) as an adjunct to opioid therapy when seeking to reduce opioid consumption in postsurgical adults admitted to the ICU (conditional recommendation, very low quality of evidence).

Rationale: Ketamine, because of its $N$-methyl-D-aspartate (NMDA) receptor-blocking properties and potential to reduce the risk for opioid hyperalgesia, has been evaluated in postoperative adults as a strategy to improve pain relief while reducing opioid requirements in two non-ICU systematic reviews $(93,94)$. In a single-center, double-blind RCT of 93 postabdominal surgery ICU patients, adjunctive ketamine $(0.5 \mathrm{mg} / \mathrm{kg}$ IV push, $2 \mu \mathrm{g} /$ $\mathrm{kg} / \mathrm{min}$ infusion $\times 24 \mathrm{hr}$ followed by $1 \mu \mathrm{g} / \mathrm{kg} / \mathrm{min} \times 24 \mathrm{hr}$ ) was 
associated with reduced morphine consumption (MD, $-22 \mathrm{mg}$; $95 \% \mathrm{CI},-30$ to -14 ; low quality) but no difference in patients' self-reported pain intensity (95). The panel noted that reduced opioid consumption is only a surrogate for better patient-centered outcomes. The incidence of side effects (i.e., nausea delirium, hallucinations, hypoventilation, pruritus, and sedation) was not different between the ketamine and opioid-alone groups. Based on this generally positive ICU RCT, the panel made a conditional recommendation for the use of low-dose ketamine as an adjunct to opioids to optimize acute postoperative pain management in critically ill adults once the benefits and harms of its use have been considered by clinicians. Because this single available ICU RCT had a high risk of bias and was also limited to postoperative abdominal surgery patients, the panel also considered indirect evidence from RCTs involving non-ICU patients that, overall, suggested benefit with ketamine use $(93,94)$.

\section{Neuropathic Pain Medications.}

Question: Should a neuropathic pain medication (e.g., gabapentin, carbamazepine, and pregabalin) be used as an adjunct to an opioid (vs an opioid alone) for pain management in critically ill adults?

Recommendations: We recommend using a neuropathic pain medication (e.g., gabapentin, carbamazepine, and pregabalin) with opioids for neuropathic pain management in critically ill adults (strong recommendation, moderate quality of evidence).

We suggest using a neuropathic pain medication (e.g., gabapentin, carbamazepine, and pregabalin) with opioids for pain management in ICU adults after cardiovascular surgery (conditional recommendation, low quality of evidence).

Rationale: Two RCTs in ICU patients with Guillain-Barré syndrome $(96,97)$ and two RCTs in postcardiac surgery ICU patients $(98,99)$ were included. Each of these trials, although double-blinded, was small and single centered. The first Guillain-Barré syndrome trial compared gabapentin (15 mg/ $\mathrm{kg} / \mathrm{d}$ ) with placebo in 18 patients using a crossover design (96). In the second Guillain-Barré syndrome trial, gabapentin $(300 \mathrm{mg} / \mathrm{d})$, carbamazepine $(100 \mathrm{mg} / \mathrm{d})$, and placebo were compared in 36 patients using a parallel design (97). Pooled analysis showed that neuropathic agents reduced pain intensity measured by the 0-10 NRS (MD, $-3.44 \mathrm{~cm} ; 95 \% \mathrm{CI},-3.90$ to -2.98 ; high quality). Patients receiving gabapentin had also significantly lower pain intensity than patients receiving carbamazepine (97). Two postcardiac surgery trials compared pregabalin (150 mg before surgery then $150 \mathrm{mg}$ daily) with placebo in 40 and 60 patients, respectively $(98,99)$.

Pooled analysis of these four trials demonstrated a significant decrease in opioid consumption in the first 24 hours after neuropathic agent initiation ( $\mathrm{MD},-13.54 \mathrm{mg}$ [morphine equivalent]; $95 \% \mathrm{CI},-14.57$ to -12.5 ; moderate quality). However, the four RCTs included diverse opioids as baseline treatment: fentanyl $(96,97)$, oxycodone (98), and tramadol (99), which may limit the applicability of results. Across the two postsurgical trials, both time to extubation $(\mathrm{MD},+0.36 \mathrm{hr}$; $95 \% \mathrm{CI},-0.7$ to +1.43 ; low quality) and ICU LOS (MD, -0.04 d; $95 \%$ CI, -0.46 to +0.38 ; low quality) were similar between the neuropathic and nonneuropathic medication groups (99).
The Guillain-Barré syndrome population is considered by neurologists to be one of the best populations to evaluate neuropathic pain medication efficacy (among the larger population of ICU patients who might have neuropathic pain). The existence of limited data and potential drawbacks to neuropathic pain medication use are distinct in the much larger population of cardiovascular surgical patients; our recommendation focuses on opiate exposure reduction in patients who, in most cases, do not have neuropathic pain. The quality of evidence for the postcardiac surgery recommendation was low due to issues related to risk of bias and imprecision (98). Panel members estimated that neuropathic agents had negligible costs and were widely available although the possible sedative and cognitive effects of these agents could preclude their use in some patients. These drugs require the ability for patients to swallow or have enteral access.

\section{Lidocaine.}

Question: Should IV lidocaine be used as an adjunct to an opioid (vs an opioid alone) for pain management in critically ill adults?

Recommendation: We suggest not routinely using IV lidocaine as an adjunct to opioid therapy for pain management in critically ill adults (conditional recommendation, low quality of evidence).

Rationale: One single-center, double-blind RCT of 100 cardiac surgery patients requiring a postoperative ICU stay found that lidocaine $(1.5 \mathrm{mg} / \mathrm{kg}$ IV bolus $\times 1$ over $10 \mathrm{~min}$ at the time of surgery followed by an IV infusion of $30 \mu \mathrm{g} / \mathrm{kg}^{\prime} \mathrm{min}$ for $48 \mathrm{hr}$ ) versus placebo did not affect patient's self-reported pain intensity; postoperative fentanyl or sedative consumption, time to extubation; nor ICU and hospital LOS when compared with placebo (100). This study had a high risk of bias related to selection bias and a lack of intention-to-treat analysis.

Evidence from non-ICU studies helped support this recommendation. A meta-analysis assessing the improvement of analgesia and opioid-related side effects in non-ICU postoperative patients reported only low-to-moderate quality evidence that adjunctive lidocaine, when compared with placebo, decreased postoperative pain intensity scores after abdominal surgery. It did not find an improvement with lidocaine use for objective outcomes like time to first spontaneous bowel movement after surgery. It did not evaluate the important safety concerns associated with lidocaine use (101). Although the use of IV lidocaine infusions as adjunctive medication is discouraged for the general ICU population, individual patients and certain surgical ICU cohorts may benefit from this intervention. Of note, the influence of IV lidocaine infusion dose and duration and interpatient pharmacokinetic variability on the risk that neurologic and cardiac toxicity will occur in the ICU population remains unclear. At this time, concerns about safety outweigh the theoretical benefits of its use in the general adult ICU population.

\section{NSAIDs.}

Question: Should a COX-1-selective NSAID be used as an adjunct to an opioid (vs an opioid alone) for pain management in critically ill adults? 
Recommendation: We suggest not routinely using a COX1-selective NSAID as an adjunct to opioid therapy for pain management in critically ill adults (conditional recommendation, low quality of evidence).

Rationale: Two single-center RCTs, one including 120 postcardiac surgery ICU patients in four parallel groups (adjunctive $75 \mathrm{mg}$ diclofenac, $100 \mathrm{mg}$ ketoprofen, $100 \mathrm{mg}$ indomethacin, or placebo) (102) and one including 43 postabdominal surgery ICU patients in two parallel groups (adjunctive $100 \mathrm{mg}$ ketoprofen or placebo) (103), evaluated the role of COX-1-selective NSAIDs for postoperative ICU pain control. Pooled analysis demonstrated that NSAIDs nonsignificantly reduced pain intensity at rest at 24 hours as measured by the $0-10$ VAS or NRS (MD, $-0.35 \mathrm{~cm} ; 95 \%$ CI, -0.91 to +0.21 ; low quality). In one trial (103), pain intensity during deep inspiration - although significantly lower at 6 hours (MD, $-1.3 \mathrm{~cm} ; 95 \% \mathrm{CI},-2.36$ to -0.24 ; moderate quality) —was not different at 24 hours (MD, $-0.6 \mathrm{~cm} ; 95 \% \mathrm{CI},-1.44$ to +0.24 ; low quality). Pooled analysis showed a significant reduction of morphine consumption at 24 hours (MD, $-1.61 \mathrm{mg}$ [morphine equivalents]; $95 \% \mathrm{CI},-2.42$ to -0.8 ; very low quality). Neither study reported a difference in nausea/vomiting between groups. No respiratory depression events were reported (103).

NSAID-related side effects including acute kidney injury and excessive bleeding were not significantly different between the three NSAIDs and the placebo group. Both studies had a high risk of bias $(102,103)$. Given the perceived small beneficial effect balanced with serious potential safety concerns (e.g., bleeding and kidney injury), particularly when NSAIDs are administered for multiple doses, the panel members recommend against routine use of NSAIDs along with opioids for nonprocedural pain management in critically ill adults. As with most conditional recommendations, the panel felt that there are likely patients-and perhaps even cohorts of patients - who may benefit from NSAIDs. No RCT evaluating a COX-2-specific NSAID (e.g., celecoxib) in critically ill adults was identified; thus, the role of these agents remains unclear.

Evidence Gaps: All adjunctive nonopioid analgesics (when used in the context of multimodal analgesia) require larger sized studies in critically ill adults that are designed to clearly evaluate their opioid-sparing properties and their ability to reduce opioid-related side effects (104). The outcomes associated with opioid safety concerns such as ileus, duration of mechanical ventilation, immunosuppression, healthcareassociated infections, delirium, and both ICU and hospital LOS must be evaluated carefully. The risks of using nonopioid-adjunctive medications for analgesia in a population at increased risk for adverse drug effects need to be better defined. This includes analysis of liver and renal toxicities secondary to acetaminophen (all routes), hemodynamic instability secondary to IV acetaminophen (85), risk of bleeding secondary to non-COX-1-selective NSAIDs, delirium, and neurotoxicity associated with ketamine (105), and hemodynamic alterations with IV lidocaine (100). The optimal dose and route of administration for these nonopioids in critically ill patients need to be investigated, and studies should be conducted in the critically ill medical patients unable to self-report pain. Finally, the role for the use of different opioid-adjunctive medications in combination needs to be evaluated.

Summary of Pharmacologic Adjuvants to Opioid Ther$a p y$. The panel generally supports the utilization of multimodal pharmacotherapy as a component of an analgesia-first approach to spare and/or minimize both opioids and sedatives. A multimodal analgesia strategy is likely to improve pain control, reduce opioid consumption, and improve patientcentered outcomes. In patients for whom the risk of these nonopioid-adjunctive medications favors their exclusion, the several nonpharmacologic strategies (described below) provide an opportunity to minimize opioid consumption.

Protocols mandating systematic assessments with validated pain and sedation scales consistently reduced the consumption of opioids and sedatives $(3,106-111)$. Studies aiming to evaluate an improvement in systematic pain assessment with validated scales evaluated cohorts in whom the use of nonopioid multimodal pharmacotherapy was significantly higher $(106,110)$. Daily sedation interruption can also be a useful intervention at reducing opioid consumption, provided proper assessment of pain precedes it (112). Music and massage, as recommended in these guidelines, have also been shown to reduce opioids (113117). Selected adjunctive agents should be both patient specific (e.g., minimizing acetaminophen use with liver dysfunction or high doses of gabapentin with renal dysfunction) and symptom specific (e.g., use of ketamine in surgical ICU patients at high risk of opioid side effects) to improve pain scores, decrease opioid consumption, minimize new adverse effects, and reduce polypharmacy (Supplemental Fig. 2 [Supplemental Digital Content 9, http://links.lww.com/CCM/D767] summarizes a pharmacologic strategy to decrease opioid consumption in the ICU).

\section{Pharmacologic Interventions to Reduce Procedural Pain}

Bedside procedures in the ICU can include regular activities (e.g., turning) and discrete procedures (e.g., arterial catheter insertion). Pain should be assessed and appropriately treated before a procedure to prevent more intense pain during the procedure. The 2013 guidelines recommended that preemptive analgesia and/or nonpharmacologic interventions (e.g., relaxation) be administered to alleviate pain in adult ICU patients before CTR and suggest these interventions before other procedures (1).

\section{Opioid Use and Dose.}

Questions: Should an opioid (vs no opioid) be used for critically ill adults undergoing a procedure?

Should a high-dose opioid (vs a low-dose opioid) be used for critically ill adults undergoing a procedure?

Recommendation: We suggest using an opioid, at the lowest effective dose, for procedural pain management in critically ill adults (conditional recommendation, moderate level of evidence).

Remarks: The same opioids (i.e., fentanyl, hydromorphone, morphine, and remifentanil) that are recommended in the 2013 guidelines to manage pain should also be considered when an opioid is deemed to be the most appropriate pharmacologic intervention to reduce procedural pain (1). 
Rationale: Three small RCTs tested the relative effectiveness of different doses of opioids administered before turning and CTR. Cardiac surgery patients in a high-dose remifentanil group versus a low-dose remifentanil group had significantly lower CTR pain (118). However, in a second study, when high-dose versus low-dose morphine was administered before turning or CTR (when steady-state morphine serum concentrations had not been reached), no significant differences in procedural pain scores were seen (119); however, procedural pain scores were low in both groups. Pooled analysis comparing high-dose versus lowdose opioids for periprocedural pain management demonstrated a small reduction in the $0-10$ NRS pain score with high-dose opioid use (standard mean difference [SMD], $-0.26 \mathrm{~cm} ; 95 \% \mathrm{CI}$, -0.94 to +0.42 ; low quality); however, conclusions are limited given the differing results between individual studies. In a third study, medical-surgical ICU patients who received IV fentanyl versus placebo before turning had a significantly lower score on the BPS (120). The potential for harm with opioids, in a dosedependent proportion, was demonstrated. Two of 20 patients in the high-dose remifentanil group had 1-3 minutes of apnea, requiring bag and mask ventilation for 3 minutes (118), whereas $10 \%$ of patients in another study who were administered highdose fentanyl (at a dose of $1-1.5 \mu \mathrm{g} / \mathrm{kg}$ ) experienced respiratory depression (120). Given this short-term consequence of higher dose opioids in critically ill patients, as well as the effectiveness of small doses of opioids in the three studies in maintaining low pain levels, opioids at the lowest effective doses for procedural pain are favored. Timing opioid administration so that the opioid's peak effect coincides with the procedure is important.

\section{Local Analgesia/Nitrous Oxide.}

Questions: Should local analgesia (vs an opioid) be used for critically ill adults undergoing a procedure?

Should nitrous oxide (vs an opioid) be used for critically ill adults undergoing a procedure?

Recommendation: We suggest not using either local analgesia or nitrous oxide for pain management during CTR in critically ill adults (conditional recommendation, low quality of evidence).

Rationale: Only one RCT tested the effects of subcutaneous infiltration of $20 \mathrm{~mL}$ of $0.5 \%$ bupivacaine around a mediastinal CTR site versus inhaled 50\% nitrous oxide and oxygen after cardiac surgery (121). Patients in the bupivacaine (vs 50\% nitrous oxide and oxygen) group had significantly lower CTR pain scores; however, the quality of evidence was low. Despite a signal of benefit, the feasibility of subcutaneous bupivacaine use in the ICU is challenging, given that it can only be administered by a qualified clinician. A lack of data to support the use of lower risk local anesthetics like lidocaine, able to be administered by a wider range of clinicians, also influenced the panel's recommendation.

\section{Volatile Anesthetics.}

Question: Should an inhaled volatile anesthetic (vs no use of this agent) be used for critically ill adults undergoing a procedure?

Recommendation: We recommend not using inhaled volatile anesthetics for procedural pain management in critically ill adults (strong recommendation, very low quality of evidence).
Rationale: Isoflurane, a volatile anesthetic, is traditionally used for general anesthesia. It has a relatively rapid onset and recovery and has demonstrated cardioprotective effects such as preserved mitochondrial oxygen consumption, troponin release, and myocardial infarction (122). Little is known of the analgesic effects of isoflurane for periprocedural pain in ICU patients.

No RCTs comparing isoflurane to a control intervention (e.g., opioid alone) were found. One small double-blinded RCT tested the relative effectiveness of nitrous oxide $50 \%$ and oxygen combined with isoflurane versus inhaled nitrous oxide $50 \%$ and oxygen alone for CTR in patients after uncomplicated cardiac surgery (123). Nitrous oxide 50\% and oxygen along with isoflurane inhalation were more effective for pain related to the first of two chest tubes removed. However, removal of the second chest tube was more painful, regardless of the gas inhaled. Although the study showed a potential for benefit, we do not recommend this intervention because the study failed to consider the CTR time relative to the gas administration time; the very low quality of evidence available (imprecision [a small sample size and only one study] and indirectness [only cardiac surgery patients]); the increased resources needed for use of gases in the ICU; and in some centers, safety issues related to the use of volatile anesthetics outside the operating room.

\section{NSAIDs.}

Question: Should an NSAID administered IV, orally, and/or rectally (vs an opioid) be used for critically ill adults undergoing a procedure?

Recommendation: We suggest using an NSAID administered IV, orally, or rectally as an alternative to opioids for pain management during discrete and infrequent procedures in critically ill adults (conditional recommendation, low quality of evidence).

Rationale: In a randomized double-blind study (124), the effects of two types of analgesics with different mechanisms of action were tested on CTR pain: a single 4-mg dose of IV morphine (an opioid) or a single 30-mg dose of IV ketorolac (a non-COX-1-specific NSAID). Procedural pain intensity scores did not differ significantly among the groups, although pain intensity was mild in both groups and the quality of evidence was limited by imprecision (small number of patients).

Question: Should an NSAID topical gel (vs no use of NSAID gel) be used for critically ill adults undergoing a procedure?

Recommendation: We suggest not using an NSAID topical gel for procedural pain management in critically ill adults (conditional recommendation, low quality of evidence).

Rationale: Topical valdecoxib is an NSAID gel. Use of a topical analgesic rather than an IV NSAID or opioid or local anesthetic injection could be less demanding on available nursing resources (125). One randomized double-blind study in postcardiac surgery patients tested the efficacy of topical valdecoxib 50-mg placebo gel (vs a paraffin gel) applied to the skin surrounding a chest tube before CTR (125). Patients who received the NSAID gel had less CTR pain than those who received the paraffin control gel. However, the panel made a 
conditional recommendation against the use of NSAID gel for procedural pain management given concerns about the quality of this study and the high acquisition cost of NSAID gel product in some countries that may make their use prohibitive.

Evidence Gaps: Future studies are warranted to test the effectiveness of various types and doses of opioids in larger sample of patients during different procedures while attending to the patients' preprocedural pain, particularly in a context where opioid exposure may be undesirable. Studies of procedural pain interventions should avoid risk of bias through use of control groups, allocation concealment, and blinding. Generalizability of study findings can be improved by including heterogeneous samples of ICU patients undergoing the same procedure and also patients admitted to the ICU with a known opioid use disorder. Much procedural pain research has used CTR as the paradigm procedure, most likely because the research protocol can be standardized more easily than with other procedures and because CTR represents a painful ICU procedure that frequently occurs after cardiac surgery. The degree to which data from CTR studies can be extrapolated to other ICU procedures likely to be associated with pain remains unclear.

\section{Nonpharmacologic Interventions to Reduce Pain Cybertherapy/Hypnosis.}

Questions: Should cybertherapy (virtual reality [VR]) (vs no use of cybertherapy) be used for pain management in critically ill adults?

Should hypnosis (vs no use of hypnosis) be used for pain management in critically ill adults?

Recommendation: We suggest not offering cybertherapy (VR) or hypnosis for pain management in critically ill adults (conditional recommendation, very low quality of evidence).

Rationale: Cybertherapy is a VR distraction postulated to reduce postoperative pain and distress in the ICU. A set of five simulated environments was displayed to the patient for 30 minutes before and after surgery (126). Hypnosis was administered by a trained ICU nurse in alert ICU patients and was induced using the cenesthesic approach (i.e., patient attention focused on any body sensation) or carried out on the actual symptom (pain or anxiety) (127). One study evaluated 67 postcardiac surgery ICU patients before and after the cybertherapy intervention (126). Most (88\%) reported a decreased level of postoperative pain (MD, $-3.75 \mathrm{~cm}$ on the $0-10$ VAS) that corresponded to a change from "severe to moderate" to "moderate to light" pain. Although risk of bias was minimal, imprecision (small sample size), failure to use a validated pain intensity scale, and the methodologic limitations inherent to observational studies led to an overall very low quality of evidence. Also, many factors related to resources (equipment, time, ICU environment, and training) make this intervention possibly infeasible to implement. Therefore, the panel suggests that clinicians not use cybertherapy for pain management in critically ill adults.

Hypnosis was evaluated with 23 burn ICU patients compared with 23 matched historical controls (127). The first ICU hypnosis session occurred at a median of 9 days (0-20 d) after injury, and an adequate level of hypnosis was obtained, on average, after 15 minutes. On the day after hypnosis, repeated pain assessments (up to 12) found that hypnosis was associated with a reduction in the $0-10$ VAS $(\mathrm{MD},-0.5 \mathrm{~cm} ; 95 \% \mathrm{CI},-1.37$ to +0.37 ; very low quality). Opioid consumption was reduced compared with historical controls. Within the intervention group, opioid consumption was lower in patients who received hypnosis at admission to the ICU compared with those who did not. The risk of bias was judged to be very serious due to poorly evaluated outcomes, variability on assessment time points, cointerventions between groups, and unclear ascertainment of exposure. Due to high risk of bias and the imprecision associated with the observational data, the overall quality of evidence was very low. Many factors (resources, ICU environment, extensive training, and patient acceptability) make this option possibly unfeasible to implement. Therefore, the panel issued a conditional recommendation against the use of hypnosis for pain management in critically ill adults.

\section{Massage.}

Question: Should massage (vs no massage) be used for pain management in critically ill adults?

Recommendation: We suggest offering massage for pain management in critically ill adults (conditional recommendation, low quality of evidence).

Remarks: Massage interventions varied in session time (10$30 \mathrm{~min}$ ), frequency (once or bid), duration (for 1-7 d), and body area (back, feet and hands, or only hands).

Rationale: Massage for postoperative ICU pain management in cardiac and abdominal surgery patients $(n=751$ and 265, respectively) was investigated in five RCTs $(65,117,128-130)$ (Supplemental Table 7, Supplemental Digital Content 10, http://links.lww.com/CCM/D768). The comparator arms were different across studies and included standard care (117, 129, $130)$, attention $(129,130)$, or sham massage (i.e., hand holding) (65). Pooled analysis showed a reduction in pain intensity scores (0-10 VAS or NRS scale) with massage use on the first day after it was provided (MD, $-0.8 \mathrm{~cm} ; 95 \% \mathrm{CI},-1.18$ to -0.42 ; low quality). Repetitive administration of massage seemed to reduce pain intensity scores with MDs varying from -0.3 to $-1.83 \mathrm{~cm}$ from day 1 to day 5 (after patients were discharged from the ICU). The overall quality of evidence was low due to risk of bias and imprecision. No adverse events were reported in relation to the administration of massage in the included studies. Resources varied across studies in which nurses or massage therapists provided the intervention. Minimal training (3-6hr) was provided to nurses. The panel felt that feasibility of using massage for ICU pain management would depend on the intervention duration and resources needed, which could affect cost.

\section{Music.}

Question: Should music therapy (vs no music therapy) be used for pain management in critically ill adults to relieve both procedural and nonprocedural pain?

Recommendation: We suggest offering music therapy to relieve both nonprocedural and procedural pain in critically ill adults (conditional recommendation, low quality of evidence). 
Rationale: Among the studies evaluated, music interventions varied in music type (participant's choice from a preselection of music or harp live music), duration (10-45 min), and pain management purposes (procedural or nonprocedural) in the evaluated studies. Participants were provided with headsets to listen to music except in one study where live harp music was played in the ICU room (116). Music interventions were administered once in most studies except in two studies in which participants received the music intervention during two turning procedures (115), and once daily up to a maximum of 3 days (117) (Supplemental Table 8, Supplemental Digital Content 11, http://links.lww.com/CCM/D769).

Effectiveness of music was tested for procedural pain management in three RCTs during different procedures including CTR in 156 cardiac surgery ICU adults (113), C-clamp procedure after percutaneous coronary interventions in 66 patients (114), and during two turning procedures in postoperative ICU patients (115). The comparator arms were different across studies and included standard care and white noise (113), headsets attached to a CD player without music (115), or a rest period (114). Pooled analysis showed that music therapy reduced pain intensity (0-10 NRS) (MD, $-0.52 \mathrm{~cm}$; 95\% CI, -1.49 to +0.45 ; low quality).

For nonprocedural pain management, effectiveness of music was tested in four studies including three RCTs with a total of 434 medical or surgical ICU patients $(12,116,117,131)$ and a pre/posttest observational study with 87 cardiac surgery ICU patients (132). The comparator arms included standard care (117) or a rest period $(116,131)$. Pooled analysis showed that music reduced pain intensity (0-10 NRS) (MD, $-0.66 \mathrm{~cm}$; 95\% CI, -0.89 to -0.43 ; low quality). These reductions in pain intensity for both procedural and nonprocedural pain management were not considered to be clinically significant. However, the potential for benefit outweighed any signal for harm or resource requirements. One large RCT that found that personal-directed music therapy reduces anxiety and sedative use in critically ill adults was not included in the evidence profile for this question because it did not report pain assessments (133).

The quality of evidence of included studies was deemed to be low (nonprocedural pain management) to very low (procedural pain management) due to risk of bias and the inconsistency in the reported results between studies. There were no reported adverse events related to music therapy. However, nine participants did not complete the music intervention in two studies because they disliked music or removed their headsets $(114,131)$. The panel felt that music is a safe intervention for pain management, but the patient's preference should be considered. Feasibility was raised as an issue by the panel depending on the resources needed for its implementation including professionals (e.g., musician and music therapist) and equipment (e.g., purchase of music and headsets). Storage room and hygiene measures must also be considered.

\section{Cold Therapy.}

Question: Should cold therapy (vs no use of cold therapy) be used for critically ill adults undergoing a procedure?
Recommendation: We suggest offering cold therapy for procedural pain management in critically ill adults (conditional recommendation, low quality of evidence).

Remarks: Cold ice packs were applied for 10 minutes, and wrapped in dressing gauze, on the area around the chest tube before its removal.

Rationale: Cold therapy for periprocedural pain management during CTR was investigated in two RCTs ( $n=130$ total) in postcardiac surgery ICU patients $(134,135)$. In one study, the effects of cold therapy were compared with usual care (i.e., oral acetaminophen every $6 \mathrm{hr}$ ) ( $n=40$ per group) (134), whereas in the other, a placebo tap water pack ( $n=25$ per group) was used as the comparator (135). Although a pooled analysis of studies demonstrated a nonsignificant reduction in pain intensity (0-10 NRS) with cold therapy (MD, $-1.91 \mathrm{~cm}$; $95 \% \mathrm{CI},-5.34$ to +1.52 ; low quality), the panel considered that a reduction of this magnitude on the NRS scale was clinically important and consistent with meaningful acute pain reductions $(1.3-2.4 \mathrm{~cm})$ as defined in one study of 700 postsurgical patients (136).

Although only CTR was investigated in a homogeneous group of postcardiac surgery patients, the panel felt that this recommendation was generalizable to other procedures and for use in other critically ill populations. No mention of possible undesirable effects related to the use of cold therapy appeared in the included literature; however, the panel agreed that these are likely to be trivial (unless the clinician forgets to remove the cold pack after CTR). Adequate room in the ICU freezer and a written protocol for use of this intervention will be required. Simple, inexpensive, and widely available interventions like cold therapy can be used frequently in resourcepoor areas where medications may not be available.

\section{Relaxation Techniques.}

Question: Should relaxation techniques (vs no use of relaxation techniques) be used for critically ill adults undergoing a procedure?

Recommendation: We suggest offering relaxation techniques for procedural pain management in critically ill adults (conditional recommendation, very low quality of evidence).

Remarks: The relaxation technique used in each study differed.

Rationale: Relaxation techniques related to breathing were tested for procedural pain management and timed with opioid administration during CTR in two different matched control studies evaluating a total of 88 postcardiac surgery ICU patients $(137,138)$. In one study (137) (in which the rapidly administered relaxation technique consisted of instructing the patient to inhale and hold their breath for a moment; to breathe out and go limp as a rag doll; and then to start yawning), the chest tube(s) were removed at the end of the yawn. In the second study (138), patients were taught breathing exercises that included inhaling slowly through the nose and exhaling slowly through pursed lips. Patients were encouraged to complete these exercises either with their eyes closed or to focus on an object in the room. Breathing exercises were initiated 5 minutes before CTR and continued during chest tube dressing, sutures, and tube removal. 
Pooled analysis showed a mean reduction in pain intensity (0-10 VAS) $15-30$ minutes after CTR (MD, $-2.5 \mathrm{~cm}$; 95\% CI, -4.18 to -0.82 ; very low quality). A reduction of this magnitude is clinically important (136). However, the quality of evidence was deemed to be very low due to the imprecision (small sample sizes) and the risk of bias. Although a breathing-focused relaxation technique was evaluated in a relatively homogeneous group of patients during only one type of painful procedure, the panel felt that this recommendation was generalizable to other painful procedures and other critically ill populations. Possible undesirable effects related to relaxation were not mentioned in the included studies, and the panel felt that these were unlikely to occur. The panel agreed that minimal resources and training were needed to provide this intervention safely and efficiently. Therefore, relaxation using breathing techniques appears feasible to implement and acceptable to stakeholders. Written information could also be provided to patients to help familiarize them with relaxation techniques.

Evidence Gaps: The effects of nonpharmacologic interventions in critically ill adults unable to self-report remain unknown. The role of a family member in the delivery of some interventions (e.g., relaxation, massage, and music) could be explored. Whether music's coanalgesic effect depends on patient's musical preferences should be considered. Interventions to reduce procedural pain should be evaluated during procedures other than CTR. Implementation studies documenting the feasibility and associated costs related to the use of these interventions are also needed. Studies to determine the effect of relaxation techniques on other outcomes such as sleep are also required.

\section{Protocol-Based Pain Assessment and Management}

Question: Should a protocol-based (analgesia/analgosedation) pain assessment and management program be used in the care of critically ill adults when compared with usual care?

Good Practice Statement: Management of pain for adult ICU patients should be guided by routine pain assessment and pain should be treated before a sedative agent is considered.

Recommendation: We suggest using an assessment-driven, protocol-based, stepwise approach for pain and sedation management in critically ill adults (conditional recommendation, moderate quality of evidence).

Remarks: For this recommendation, analgosedation is defined as either analgesia-first sedation (i.e., an analgesic [usually an opioid] is used before a sedative to reach the sedative goal) or analgesia-based sedation (i.e., an analgesic [usually an opioid] is used instead of a sedative to reach the sedative goal). The implementation of this recommendation infers that institutions should have an assessment-driven protocol that mandates regular pain and sedation assessment using validated tools, provides clear guidance on medication choice and dosing, and makes treating pain a priority over providing sedatives.

Rationale: The five outcomes deemed critical to the recommendation include pain intensity, medication exposure (analgesics/sedatives), adverse events, duration of mechanical ventilation, and ICU LOS $(5,106-110,127,139-156)$ (Supplemental Table 9, Supplemental Digital Content 12,
http://links.lww.com/CCM/D770). Pooled analysis suggests that a protocol-based (analgesia/analgosedation) pain and sedation assessment management program compared with usual care does not affect the incidence of nosocomial infection, constipation, hypotension, bradycardia, or opioid exposure, but does reduce sedative requirements (SMD, -0.57 ; $95 \% \mathrm{CI},-0.84$ to -0.31 ; low quality), duration of mechanical ventilation (MD, $-1.26 \mathrm{~d}$; $95 \% \mathrm{CI},-1.8$ to -0.73 ; moderate quality), ICU LOS (MD, $-2.27 \mathrm{~d}$; $95 \% \mathrm{CI},-2.96$ to -1.58 ; moderate quality), and pain intensity (0-10 VAS or NRS) (MD, $-0.35 \mathrm{~cm} ; 95 \% \mathrm{CI},-0.22$ to -0.49 ; low quality). Panel members issued a conditional recommendation because the benefits of a protocol-based approach were not observed across all critical outcomes.

Evidence Gaps: To be able to generate strong recommendations for the use of a protocol-based analgesia/analgosedation program, future randomized studies must be completed that address the following questions: 1 ) what is the optimal opioid, or other analgesic, to use in the protocol? 2) what ICU setting or patient population is most appropriate for the use of such a protocol? 3) what are the potential benefits of such protocols based on their ability to reduce pain or avoid the use of potentially harmful effects of sedatives? and 4) what are the potential safety concerns associated with such protocols (e.g., opioid withdrawal, posthospital opioid use disorder)?

\section{AGITATION/SEDATION}

Sedatives are frequently administered to critically ill patients to relieve anxiety, reduce the stress of being mechanically ventilated, and prevent agitation-related harm (1). These medications may predispose patients to increased morbidity (157, 158). The healthcare provider must determine the specific indication for the use of sedatives. If a sedative is needed, the patient's current sedation status should be assessed and then frequently reassessed using valid and reliable scales (158-161). In critically ill patients, unpredictable pharmacokinetics and pharmacodynamics secondary to drug interactions, organ dysfunction, inconsistent absorption and protein binding, hemodynamic instability, and drug accumulation can lead to adverse events $(1,162,163)$.

The 2013 guidelines (1) suggested targeting light levels of sedation or using daily awakening trials (112, 164-166), and minimizing benzodiazepines (167) to improve short-term outcomes (e.g., duration of mechanical ventilation and ICU LOS). In addition, sedation delivery paradigms and the specific sedative medication used can have an important impact on postICU outcomes including 90-day mortality physical functioning, neurocognitive, and psychologic outcomes. These issues have been evaluated in the present guidelines through three actionable and three descriptive questions. (A prioritized topic list is in Supplemental Table 10 [Supplemental Digital Content 13, http://links.lww.com/CCM/D771], and voting results appear in Supplemental Table 11 [Supplemental Digital Content 14, http:// links.lww.com/CCM/D772].) The evidence summaries and evidence-to-decision tables used to develop recommendations 
for the agitation (sedation) group are available in Supplemental Table 12 (Supplemental Digital Content 15, http://links.lww. $\mathrm{com} / \mathrm{CCM} / \mathrm{D} 773$ ), and the forest plots for all completed metaanalyses are available in Supplemental Figure 3 (Supplemental Digital Content 16, http://links.lww.com/CCM/D774).

\section{Light Sedation}

Question: Does light sedation (vs deep sedation), regardless of the sedative agent(s) used, significantly affect outcomes in critically ill, mechanically ventilated adults?

Recommendation: We suggest using light sedation (vs deep sedation) in critically ill, mechanically ventilated adults (conditional recommendation, low quality of evidence).

Rationale: The 2013 PAD guidelines made an ungraded statement that maintaining a light level of sedation will shorten time to extubation and reduce ICU LOS (1). Although the prior guideline defined light sedation as a RASS scale score of greater than or equal to -2 and eye opening of at least 10 minutes (112), this level of sedation is probably deeper than required for management of mechanically ventilated adults in an ICU. No universally accepted definition of light sedation exists. To address this question, we evaluated studies in which light versus deep sedation were defined a priori, measured, and explicitly reported with objective scales describing whether patients met these clear light, versus deep, sedation targets systematically over the time spent in the ICU and at least q6h. Surrogate measures (e.g., sedative plasma levels) or subjective clinical assessments of wakefulness were not considered as part of the definition of level of sedation. Studies describing a daily spontaneous awakening trial (SAT) were not deemed indicative of a light sedation approach because they reported lightening of sedation at a single point in time, rather than over the entire day. For studies that used scales, such as the RASS (159), a RASS score of -2 to +1 range (or its equivalent using other scales) was considered as light sedation in the evaluated studies.

Eight RCTs satisfied our research criteria $(156,168-174)$. We evaluated the effect of light versus deep sedation on outcomes that were considered critical by the sedation group and patient representatives: 90-day mortality, time to extubation, delirium, tracheostomy, cognitive and physical functional decline, depression, and posttraumatic stress disorder (PTSD). The outcomes evaluated were mostly measured after ICU discharge and are different from the short-term outcomes assessed in the 2013 guideline ungraded descriptive question. Light sedation was not associated with 90-day mortality (RR, 1.01; 95\% CI, $0.80-1.27$; moderate quality) $(168,169)$, but it was associated with a shorter time to extubation (MD, $-0.77 \mathrm{~d}$; $95 \% \mathrm{CI},-2.04$ to -0.50 ; low quality) (168-170) and a reduced tracheostomy rate (RR, 0.57 ; 95\% CI, $0.41-0.80$; low quality) (170, 171). Light sedation was not associated with a reduction in the incidence of delirium (RR, 0.96 ; 95\% CI, 0.80-1.16; low quality) (168, 172), PTSD (RR, 0.67; 95\% CI, 0.12-3.79; low quality) (156, 174 ), depression (RR, 0.76 ; $95 \% \mathrm{CI}, 0.10-5.58$; very low quality) $(156,170)$, or self-extubation (RR, 1.29; $95 \%$ CI, 0.58-2.88; low quality) (168-170, 173). No RCTs evaluated the impact of light versus deep sedation on cognitive or physical functioning.
The overall quality of the body of evidence was low. Both the magnitude of reduction in time to extubation and tracheostomy rate were considered small; the magnitude of harm associated with self-extubation was uncertain. We initially evaluated the data from RCTs and then reviewed observational studies related to outcomes where the RCT data were of low quality. Observational trials suggested benefits in reduced risk of death at 90 days and time to extubation, but not in delirium outcomes $(166,175,176)$. One recent cohort study not considered in the guideline evidence demonstrates that sedation intensity (sum of negative RASS measurements by number of assessments) independently, in an escalating dose-dependent relationship, predicts increased risk of death, delirium, and delayed time to extubation (177). The amount of sedation preferred by patients is likely variable; some patients or families may prefer deeper sedation, but this preference may not be considered appropriate by clinicians given the adverse outcomes associated with deep sedation. Uncertainty about the cost-effectiveness of light sedation was considered. Light sedation was considered likely acceptable to clinicians and patients and feasible to implement.

Evidence Gaps: Despite the wide use of validated sedation scales, no consensus on the definition of light, moderate, and deep sedation is available. Further exploration of the concept of wakefulness and light sedation is required. The relationship between changing levels of sedation and their duration over the course of the ICU stay and clinical outcomes is also unknown. The effect of depth of sedation on post-ICU, patient-centered outcomes such as 90-day all-cause mortality and cognitive function, physical recovery, PTSD, anxiety, and depressive symptoms has not been well evaluated in RCTs. There is also a dearth of information regarding the interaction among sedative choice, sedation depth, and the patient-specific factors that affect this relationship. Finally, as outlined elsewhere in these guidelines, the relationship between level of sedation and the ability to evaluate, pain, delirium, and sleep has not been fully elucidated.

\section{Daily Sedative Interruption/Nurse-Protocolized Sedation}

Question: In critically ill, intubated adults, is there a difference between daily sedative interruption (DSI) protocols and nursing-protocolized (NP)-targeted sedation in the ability to achieve and maintain a light level of sedation?

Ungraded Statement: In critically ill, intubated adults, DSI protocols and NP-targeted sedation can achieve and maintain a light level of sedation.

Remarks: A DSI or a SAT is defined as a period of time, each day, during which a patient's sedative medication is discontinued and patients can wake up and achieve arousal and/or alertness, defined by objective actions such as opening eyes in response to a voice, following simple commands, and/or having a Sedation-Agitation Scale (SAS) score of 4-7 or a RASS score of -1 to +1 . NP-targeted sedation is defined as an established sedation protocol implemented by nurses at the bedside to determine sedative choices and to titrate these medications to achieve prescription-targeted sedation scores. 
Rationale: Five randomized, prospective, unblinded trials compared DSI protocols and NP-targeted sedation to usual care (178-182) (Supplemental Table 13, Supplemental Digital Content 17, http://links.lww.com/CCM/D775). Some studies compared DSI to "usual care," defined as an NP protocol. Most studies did not specifically target or assess how effectively either technique achieved light level of sedation; rather, they evaluated the differences in the overall sedation scores among patients being managed with DSI or NP-targeted sedation. Across the five studies, a total of 739 patients were randomized (DSI, $n=$ 373; NP, $n=366$ ). Benzodiazepines were commonly prescribed for sedation in both groups, often paired with opioids for analgesia. Two studies reported no difference in level of sedation achieved between DSI and NP-targeted sedation (178, 179). The remaining studies appear contradictory; one noted higher RASS with DSI versus NP-targeted sedation (180), another noted lower median SAS scores with DSI versus NP-targeted sedation, but no difference in the percentage of time spent in the targeted light sedation range (181). A third study reported lighter sedation with DSI than with NP-targeted sedation (182).

As outlined in these guidelines, clinicians should target a light rather than deep level of sedation in their intubated, critically ill adult patients unless deeper sedation is clinically indicated. Our literature review suggests that both DSI protocols and NP-targeted sedation are safe and no differences exist between them in achieving and maintaining a light level of sedation. There are, however, some important caveats: first, most studies evaluating DSIs and NP have done so in the context of sedation with benzodiazepines, which are no longer recommended for sedation in critically ill patients; second, DSI protocols may be associated with increasing nursing workload (179); and third, a brief DSI should not be used to justify the use of deep sedation for the rest of the day when it is not indicated. Because light levels of sedation are associated with improved outcomes and are needed to facilitate other interventions such as spontaneous breathing trials and early mobilization, healthcare providers should strive to achieve light levels of sedation in the majority of patients the majority of the time. Light sedation, assessed using a validated sedation scale, can be achieved either using a NP or through DSI protocols (where light sedation is targeted, whereas sedatives are infusing).

Evidence Gaps: Variability in nursing sedation assessment frequency and its reporting, and modality of sedative administration (infusion vs bolus) differ among institutions. The most frequent sedative choice (benzodiazepines) described in the studies may not reflect current practice. Patient and family preferences and education as to depth of sedation within a "light sedation" range should also be considered. Nonetheless, future research should focus on the effect of sedation level on patient-centered outcomes.

\section{Choice of Sedative}

Critically ill adults may require sedation to reduce anxiety and stress and to facilitate invasive procedures and mechanical ventilation. Sedation indication, goal, clinical pharmacology, and acquisition cost are important determinants in choosing a sedative agent. The 2013 PAD guidelines suggest (in a conditional recommendation) that nonbenzodiazepine sedatives (either propofol or dexmedetomidine) are preferable to benzodiazepine sedatives (either midazolam or lorazepam) in critically ill, mechanically ventilated adults because of improved shortterm outcomes such as ICU LOS, duration of mechanical ventilation, and delirium (1). For the current guidelines, we considered both short-term and long-term outcomes as critical for evaluation. These included time to extubation, time to light sedation, and delirium, and long-term outcomes such as 90-day mortality, cognitive and physical functioning, institutionalization, and psychologic dysfunction.

Elective cardiac surgical patients are different from critically ill medical and surgical patients whose admission profile is seldom elective and whose ICU stay and mechanical ventilation duration are longer. We therefore separated studies describing mechanically ventilated, routine cardiac surgical patients and critically ill, mechanically ventilated medical and surgical patients. Pharmacogenomic factors that may influence the response of sedatives and other medications in the critically ill were reviewed (163).

\section{Cardiac Surgery}

Question: Should propofol, when compared with a benzodiazepine, be used for sedation in mechanically ventilated adults after cardiac surgery?

Recommendation: We suggest using propofol over a benzodiazepine for sedation in mechanically ventilated adults after cardiac surgery (conditional recommendation, low quality of evidence).

Rationale: We identified eight RCTs: seven of which compared infusions of both sedative agents (183-189) and one RCT compared propofol infusions to midazolam boluses (190). In cardiac surgical patients, we considered a shortened time to light sedation of at least 30 minutes and time to extubation of at least 1 hour to be clinically significant. Two small RCTs $(n=$ 70) reported shorter time to light sedation with propofol when compared with benzodiazepines (MD, $-52 \mathrm{~min}$; $95 \% \mathrm{CI},-77$ to -26 ; low quality) $(185,186)$. Seven RCTs $(n=409)$, including one study using only benzodiazepine boluses reported shorter time to extubation with propofol versus a benzodiazepine (MD, $-1.4 \mathrm{hr} ; 95 \% \mathrm{CI},-2.2$ to -0.6 ; low quality) (183-189). We were unable to find RCTs comparing propofol and benzodiazepine effects on other critical outcomes in the cardiac surgical population. Overall, the panel judged that the desirable consequences of using propofol probably outweigh the undesirable consequences, and thus issued a conditional recommendation favoring propofol over a benzodiazepine.

\section{Medical and Surgical Patients Not Undergoing Cardiac Surgery}

Questions: Should propofol, when compared with a benzodiazepine, be used for sedation in critically ill, mechanically ventilated adults?

Should dexmedetomidine, when compared with a benzodiazepine, be used for sedation in critically ill, mechanically ventilated adults? 
Should dexmedetomidine, when compared with propofol, be used for sedation in critically ill, mechanically ventilated adults?

Recommendation: We suggest using either propofol or dexmedetomidine over benzodiazepines for sedation in critically ill, mechanically ventilated adults (conditional recommendation, low quality of evidence).

Rationale: We evaluated the effect of propofol versus benzodiazepine, dexmedetomidine versus benzodiazepine, and propofol versus dexmedetomidine in three separate analyses for the outcomes deemed critical. In most studies, benzodiazepines were administered as continuous infusions and not intermittent boluses. We combined studies using midazolam and lorazepam. In critically ill, mechanically ventilated patients, a shortened time to light sedation of at least 4 hours and time to extubation of at least 8-12 hours (one nursing shift) were deemed clinically significant.

Propofol Versus Benzodiazepines. Seven trials ( $n=357$ ) (191197) reported shorter time to light sedation with propofol when compared with a benzodiazepine (MD, $-7.2 \mathrm{hr} ; 95 \% \mathrm{CI},-8.9$ to -5.5 ; low quality). Nine trials $(n=423)(191,196-202)$ reported shorter time to extubation with propofol compared with a benzodiazepine (MD, $-11.6 \mathrm{hr}$; 95\% CI, -15.6 to -7.6 ; low quality). Only one RCT assessed delirium and found no difference (196). No data were available for other critical outcomes. Although propofol was associated with a higher risk of self-extubation (RR, $2.2 ; 95 \%$ CI, 0.30-26.45; low quality), reliable conclusions for this outcome cannot be made given the wide CI. Additionally, it was not clear if the self-extubations caused any harm (e.g., need for reintubation). Although this was an important consideration for the physicians on the sedation group panel, ICU patients might feel otherwise. Overall, the panel judged that the desirable consequences of using propofol probably outweighs the undesirable consequences, and thus issued a conditional recommendation favoring propofol over a benzodiazepine infusion.

Dexmedetomidine Versus Benzodiazepines. Five RCTs $(n=1,052)$ assessed duration of mechanical ventilation $(167,172$, 202-204); three studies $(n=969)$ evaluated ICU LOS $(167,172$, $203)$; and four RCTs $(n=1,007)$ evaluated delirium prevalence $(167,172,203,205)$. The study with the lowest risk of bias ( $n=$ 366), Safety and Efficacy of Dexmedetomidine Compared With Midazolam (SEDCOM), had the greatest benefit for the time to extubation (MD, $-1.90 \mathrm{~d}$; $95 \% \mathrm{CI},-2.32$ to -1.48 ) and delirium (RR, 0.71; 95\% CI, 0.61-0.83) with dexmedetomidine compared with a benzodiazepine infusion, and influenced how the evidence was graded when developing this recommendation (167).

Although the study by $\mathrm{Xu}$ et al (205) also showed reduced delirium with dexmedetomidine use, and the Dexmedetomidine Versus Midazolam for Continuous Sedation in the ICU (MIDEX) study (203) demonstrated a shorter duration of mechanical ventilation with dexmedetomidine over a benzodiazepine infusion, pooled analysis of all evaluated studies did not show a significant benefit of dexmedetomidine compared with a benzodiazepine infusion for duration of mechanical ventilation extubation (MD, $-0.71 \mathrm{~d}$; $95 \% \mathrm{CI}$, -1.87 to 0.45 ; low quality), ICU LOS (MD, $-0.23 \mathrm{~d}$; $95 \% \mathrm{CI}$,
-0.57 to 0.11 ; low quality), and the risk for delirium (RR, 0.81 ; 95\% CI, 0.60-1.08; low quality). Of note, the MIDEX study (203), in which delirium was assessed only once 48 hours after sedation discontinuation, showed no improvements in delirium prevalence with dexmedetomidine.

The SEDCOM (167) and Maximizing the Efficacy of Sedation and Reducing Neurological Dysfunction (MENDS) (172) studies both demonstrated a greater incidence of bradycardia in the dexmedetomidine group; neither study found intervention was required for the bradycardia. Overall, the panel judged that the desirable consequences of using dexmedetomidine probably outweigh any undesirable consequences and thus issued a conditional recommendation favoring dexmedetomidine over a benzodiazepine.

Propofol Versus Dexmedetomidine. Three RCTs $(n=850)$ assessed time to extubation and showed no difference in this outcome $(202,203,206)$. No data were available for other critical outcomes. A single RCT, the Propofol Versus Dexmedetomidine for Continuous Sedation in the ICU (PRODEX) study, showed a decreased incidence of delirium with dexmedetomidine at the single time point of 48 hours after sedation cessation (203). Patients were able to communicate more effectively if sedated with dexmedetomidine when compared with propofol (203). No differences were reported in bradycardia or hypotension between patients sedated with propofol and dexmedetomidine (203).

Overall, there was low quality evidence for the outcomes assessed, with a moderate benefit noted (reduced time to light sedation and extubation) when both propofol and dexmedetomidine were compared with benzodiazepines. No important differences in outcomes were noted between propofol and dexmedetomidine. As reported in these studies, associated harm with either propofol or dexmedetomidine was deemed to be minimal and not clinically significant. The cost-effectiveness of these sedative regimens was uncertain as both propofol and dexmedetomidine acquisition costs are now lower than when they were initially studied. Additionally, the cost of acquisition of these agents varies widely in the world, making it difficult to generalize cost-effectiveness. Nevertheless, incorporating both propofol and dexmedetomidine into practice was likely acceptable and feasible. Recognizing that dexmedetomidine should not be used when deep sedation (with or without neuromuscular blockade) is required, panel members judged that the desirable and undesirable consequences of using propofol (vs dexmedetomidine) were balanced; therefore, they issued a conditional recommendation to use either agents for sedation of critically ill adults. Implementation will likely depend on the availability of the drug and its associated cost at individual institutions.

Evidence Gaps: Larger, well-conducted studies assessing the critical outcomes we defined need to be undertaken. Faster extubation and increased hospital survival, though the building blocks of long-term outcomes, no longer suffice as the sole descriptors of patient-centered outcomes. Improvements in many aspects of survivorship, including return to former quality of life, independent function, and employment, are meaningful (207). Further studies evaluating the value of patient communication with family members during and after ICU 
care and the perceptions of patients while on each of these sedatives are also needed; of note, our patient panel members described very different subjective experiences when receiving sedatives that could not be translated into guideline recommendation content. Pharmacokinetic and pharmacodynamic considerations should be incorporated in both sedative choice and delivery methods $(162,163)$. For example, the risks and benefits of an intermittent benzodiazepine administration strategy after establishing analgesia need to be studied against use of continuous sedative infusions. Benzodiazepine medications still form the mainstay of therapy in resource-poor areas; risks and benefits need to be studied in the context of their cost. Additionally, the role of sedative medications in the context of an analgesia-first approach or to supplement analgosedation needs to be better studied. The role of benzodiazepines versus propofol or dexmedetomidine in patients who are hemodynamically unstable, need deep sedation, are at risk for delirium, or have signs of alcohol withdrawal needs to be studied. With increased propofol use, strategies to detect propofol-related infusion syndrome earlier are required and large-scale registry studies to characterize its prevalence and risks should be undertaken. The role of nonpharmacologic strategies to reduce agitation, anxiety, and distress in terms of sedative choice and requirements is uncertain, and thus, no recommendations could be made in this regard.

\section{Objective Sedation Monitoring}

Question: Are objective sedation monitoring tools (electroencephalogram-based tools or tools such as HR variability, actigraphy, and evoked potentials) useful in managing sedation in critically ill, intubated adults?

Ungraded Statements: Bispectral index (BIS) monitoring appears best suited for sedative titration during deep sedation or neuromuscular blockade, though observational data suggest potential benefit with lighter sedation as well.

Sedation that is monitored with BIS compared with subjective scales may improve sedative titration when a sedative scale cannot be used.

Rationale: The literature for ICU-based studies of objective monitoring tools for sedation consists primarily of reports for electroencephalogram-based tools (particularly the BIS). Few ICU-based studies evaluated outcome benefits (208-210). The methods used to evaluate the accuracy of BIS in the ICU are outlined in Supplemental Table 14 (Supplemental Digital Content 18, http://links.lww.com/CCM/D776), and the characteristics of the 32 studies included are summarized in Supplemental Table 15 (Supplemental Digital Content 19, http://links.lww.com/CCM/D777) (161, 208-239).

Several common challenges in research design for these studies have been identified. The relationship between electroencephalogram data and subjective sedation data was often assumed to be constant and linear, but this is an inaccurate perception. Because sedation gets deeper and patients become unresponsive, subjective sedation scales reach a minimum value (SAS 1 or RASS -5 ), whereas objective electroencephalogram-based tools can continue to decline until an isoelectric electroencephalogram is obtained (Supplemental Fig. 4, Supplemental Digital Content 20, http://links.lww.com/CCM/ D778) (211). At the other extreme, with increasing agitation, objective tools reach a maximum (i.e., a BIS 100), whereas subjective scales continue to describe increasing levels of agitation (Supplemental Fig. 5, Supplemental Digital Content 21, http://links.lww.com/CCM/D779) (211). In addition, objective monitors such as BIS allow measurement without stimulating the patient, whereas subjective sedation scales require assessing the patient response to voice, physical, and even noxious stimuli. This stimulation changes the preexisting state of the patient and increases the BIS value; depending on the timing of the BIS measurements (i.e., before, during, or after stimulation), agreement between the two assessment techniques will be affected.

The 32 ICU-based studies that compared BIS and subjective sedation scale assessment were scored based on their approach to timing of BIS measurement relative to the stimulation from subjective assessment ( $0-4$ points), type of stimulation (0-2 points), adjustment for deep sedation ( $0-2$ points), and whether electroencephalogram signal quality and software version were defined (0-2 points) $(161,208-239)$. Studies with less potential confounding (4 points on the timing issue) trended to better agreement between BIS and subjective scales $(p=0.09)$, whereas the studies that did not account for the effect of subjective stimulation (scoring 0 on timing) had the worst agreement between BIS and subjective scales (see the red ellipse in Supplemental Fig. 6, Supplemental Digital Content 22, http://links.lww.com/CCM/D780).

Three studies evaluated the effect of using the BIS to assess sedation compared with using a subjective tool (209-211). These showed reductions in total sedative use and faster wakening times despite similar clinical sedation (Ramsay 4) (208), a reduction in procedure-related adverse events (Ramsey 2-3) (209), and reduced midazolam and fentanyl doses, less agitation, less need for tracheostomy, and shorter ICU LOS (210).

Evidence Gaps: Research methodology to evaluate ICU sedation monitors has not been standardized, resulting in wide variability in study design as noted above. Defining best components and approaches will improve study quality. With improved research rigor, valid comparisons between the various objective sedation monitoring tools and between objective and subjective sedation scales may be possible. Additional research is needed to define the best approach to dealing with issues such as depth of sedation (particularly in an era when more patients are lightly sedated), stimulation during sedation assessment, and how different patient pathology (neurologic vs nonneurologic diagnoses) may affect objective tool reliability. Finally, more outcome studies are needed to confirm whether these tools improve patient outcomes or reduce healthcare resource consumption compared with subjective scales.

\section{Physical Restraints}

Question: What are the prevalence rates, rationale, and outcomes (harm and benefit) associated with physical restraint use in intubated or nonintubated critically ill adults? 
Ungraded Statements: Physical restraints are frequently used for critically ill adults although prevalence rates vary greatly by country.

Critical care providers report using restraints to prevent self-extubation and medical device removal, avoid falls, and to protect staff from combative patients despite a lack of studies demonstrating efficacy and the safety concerns associated with physical restraints (e.g., unplanned extubations and greater agitation).

Rationale: In an era focused on improving patient-centered care, the effect physical restraints have on the care and outcomes of critically ill adults remains controversial. Physical restraints are defined as "any manual method, physical, or mechanical device, material, or equipment that immobilizes or reduces the ability of a patient to move his or her arms, legs, body, or head freely" (240). This question specifically focuses on physical restraints attached to the ankle, wrist, or upper torso. Physical restraint use varies widely from $0 \%$ in some European countries to more than $75 \%$ in North America (Supplemental Table 16, Supplemental Digital Content 23, http://links.lww. com/CCM/D781) (168, 241-261). The type and location (e.g., wrist, ankle, upper torso) of physical restraints similarly vary, with resource-rich countries reporting using commercially available restraints $(242,245-247,249,252,255,260,262-268)$.

Healthcare providers have historically justified the use of physical restraints in the ICU for many reasons including to enhance patient safety $(242,249,252,262,263)$; prevent selfextubation, tube dislodgement, and/or medical device removal $(242,246,249,255,262,263,265,266,269)$; control patient behavior $(249,262,265,266,269)$; protect staff from combative patients (263); and prevent falls $(242,263,266)$. Less commonly cited reasons include the following: preserving posture/ positioning of the patient $(249,266)$; staffing shortages or lack of supervision during break coverage $(249,263,265)$; and compliance with patient, family member, or other medical staff suggestions (265).

To date, no RCT has explored the safety and efficacy of physical restraint use in critically ill adults. The few descriptive studies exploring physical restraint use and outcomes of the critically ill paradoxically report higher rates of the events that their use is intended to prevent. These events include more unplanned extubations and frequent reintubations $(245,247$, 267, 268); greater unintentional device removal (268); longer ICU LOS (245); increased agitation; higher benzodiazepine, opioid, and antipsychotic medication use $(244,268)$; and increased risk for delirium or disorientation $(257,259,268$, 270, 271).

Certain modifiable and nonmodifiable factors appear to increase critically ill adults' risk for physical restraint use. These factors include the following: older age $(250,264)$; non-coma level of arousal; neurologic or psychiatric conditions including delirium $(257,258,261,268)$; sedative type/ strategy $(169,242,261,272)$; mechanical ventilation use (242, $261,263)$; use of invasive devices $(246,250)$; nurse-to-patient ratio and perceived workload $(242,268,271)$; and time of day (249). Interestingly, patients participating in an early mobility program (273) who received early pharmacologic treatment of delirium (272) and patients who had a history of alcohol use were less restrained (268).

Patients' perceptions of being physically restrained during an ICU stay vary but often provoke strong emotional responses that persist after the ICU stay $(169,269)$. Given the prevalence, unintended consequences, and patients' perceptions of physical restraint use, critical care providers should closely weigh the risks and benefits of this practice in the adult ICU setting before initiating or maintaining physical restraint use. Although certain countries report a "restraint-free" ICU environment, it may be possible that their use of bedside sitters and/or pharmacologic restraints is increased.

Evidence Gaps: Whether efforts to reduce physical restraint use will have the unintended consequence of increasing patients' exposure to potentially harmful sedative and antipsychotic medications remain unclear. The effect nurse staffing patterns, staff education, and patient/family advocacy have on the incidence of physical restraint use in the ICU has also yet to be determined. Particularly relevant to the ICU setting, the necessity and ethics of physical restraints during end-of-life care need further exploration. Finally, the true effect physical restraints play on outcomes relevant to patients should be explored in RCTs.

\section{DELIRIUM}

Delirium is common in critically ill adults. The delirium encountered in the ICU and other settings are assumed to be equivalent pathophysiologic states. Delirium is a clinical diagnosis; most studies detect delirium using screening tools such as the Confusion Assessment Method for the ICU (CAM-ICU) or the Intensive Care Delirium Screening Checklist (ICDSC) $(274,275)$. Delirium can be disturbing for affected patients and relatives and is associated with worse outcome, and much higher ICU and hospital LOS and costs (276). Many research gaps exist in this area (277). In this guideline, we address six actionable questions and five descriptive questions (see prioritized topic list in Supplemental Table 17 [Supplemental Digital Content 24, http://links.lww.com/CCM/D782] and voting results in Supplemental Table 18 [Supplemental Digital Content 25, http://links.lww.com/CCM/D783]). The evidence summaries and evidence-to-decision tables used to develop recommendations for the delirium group are available in Supplemental Table 19 (Supplemental Digital Content 26, http:// links.lww.com/CCM/D784), and the forest plots for all metaanalyses are available in Supplemental Figure 7 (Supplemental Digital Content 27, http://links.lww.com/CCM/D785).

\section{Risk Factors}

Question: Which predisposing and precipitating risk factors are associated with delirium occurrence (i.e., incidence, prevalence, or daily transition), delirium duration, or severity in critically ill adults?

Ungraded Statement: For the following risk factors, strong evidence indicates that these are associated with delirium in critically ill adults: "modifiable"-benzodiazepine use and blood transfusions, and "nonmodifiable"-greater 
age, dementia, prior coma, pre-ICU emergency surgery or trauma, and increasing Acute Physiology and Chronic Health Evaluation (APACHE) and ASA scores.

Rationale: Sixty-eight studies published from 2000 to November 2015 that evaluated critically ill adults not undergoing cardiac surgery for delirium that used either multivariable analysis or randomization were used to evaluate variables as potential risk factors (Supplemental Table 20, Supplemental Digital Content 28, http://links.lww.com/CCM/D786). Risk of bias of the retrieved articles was scored (cohort studies using the Scottish Intercollegiate Guidelines Network quality checklist[s] and controlled trials using Cochrane methods), and studies were classified as high, acceptable, or low quality (Supplemental Table 21, Supplemental Digital Content 29, http://links.lww. com/CCM/D787). Each variable was evaluated using three criteria: 1) the number of studies investigating it; 2) the quality of these investigations, and 3) where consistency existed across the studies (i.e., the direction of association was consistent for $\geq 50 \%$ of studies). Strengths of association were not summarized because of the heterogeneity between studies. The following, nonvalidated, criteria were used to define whether there was strong, moderate, or inconclusive evidence that a risk factor was associated with increased delirium: strong-more than or equal to two high-quality articles and association consistency; moderate-one high-quality article and more than or equal to one acceptable quality article with association consistency; and inconclusive - inconsistent findings and no fulfilment of criteria for strong evidence and for moderate evidence (278). The evaluation of predisposing and precipitating risk factors was combined because these were studied in most investigations simultaneously.

Benzodiazepine use and blood transfusion administration are the only two modifiable factors with strong evidence for an association with delirium detected by screening tools (Supplemental Table 22, Supplemental Digital Content 30, http://links.lww. com/CCM/D788). The nonmodifiable risk factors with strong evidence for an association with delirium include increasing age, dementia, prior coma, pre-ICU emergency surgery or trauma, and increasing APACHE and ASA scores. Sex, opioid use, and mechanical ventilation each have been strongly shown not to alter the risk of delirium occurrence. Moderate evidence exists showing the following increase the risk for delirium: history of hypertension; admission because of a neurologic disease; trauma; and the use of psychoactive medication (e.g., antipsychotics, anticonvulsants). A history of respiratory disease, medical admission, nicotine use, dialysis or continuous venovenous hemofiltration, and a lower Glasgow Coma Scale score have each been moderately shown not to increase the risk for delirium. See the "Sedation section" for a review on how sedative choice may affect delirium and the "Sleep section" regarding the relationship between sleep and delirium. For all other potential delirium-associated risk factors, evidence currently remains inconclusive.

\section{Prediction}

Question: Can delirium be predicted in critically ill adults?

Ungraded Statement: Predictive models that include delirium risk factors at both the time of ICU admission and in the first 24 hours of ICU admission have been validated and shown to be capable of predicting delirium in critically ill adults.

Rationale: We identified four studies that used modeling to predict ICU delirium (279-282), three of which were considered to be psychometrically strong (Supplemental Table 23, Supplemental Digital Content 31, http://links.lww.com/ CCM/D789) (280-282). Of these, two studies aimed to predict ICU delirium within 24 hours after ICU admission using the PREdiction of DELIRium in ICu patients (PRE-DELIRIC) model $(280,281)$. In a multinational study, 10 predictors (age, APACHE-II score, admission group, urgent admission, infection, coma, sedation, morphine use, urea level, and metabolic acidosis) permitted a model with an area under the receiver operating characteristic (AUROC) curve of 0.77 (95\% CI, 0.74$0.79)$ (281). In another high-quality, multinational study (282), a model was built to predict delirium with patient characteristics available at ICU admission. This Early (E)-PRE-DELIRIC model includes nine predictors (age, history of cognitive impairment, history of alcohol abuse, blood urea nitrogen, admission category, urgent admission, mean arterial BP, use of corticosteroids, and respiratory failure) and was found to have an AUROC of 0.76 (95\% CI, 0.73-0.77). Because both the PRE-DELIRIC and the E-PRE-DELIRIC models had similar predictive value, the model of choice can be based on availability of predictors (Supplemental Table 24, Supplemental Digital Content 32, http://links.lww.com/CCM/D790). Both models were based on screening with the CAM-ICU only.

Evidence Gaps: Future etiologic studies on delirium should focus on presumed risk factors for which there is currently inconclusive evidence and where modifiability is likely. The effect of a reduction in known delirium risk factors including comorbid diseases, sepsis, nicotine and alcohol abuse, and the use of opioids and systemic steroids on delirium burden and patient outcome is unknown. Confounding is a key issue in these studies. Future studies on delirium risk factors should therefore make adequate adjustments based on previously considered risk factors (278).

\section{Assessment}

Question: Should we assess for delirium using a valid tool (compared with not performing this assessment with a valid tool) in critically ill adults?

Good Practice Statement: Critically ill adults should be regularly assessed for delirium using a valid tool.

Remarks: The previous guidelines provided psychometric appraisals of pain, sedation, and delirium screening tools (1). A reevaluation of the psychometrics for available delirium screening tools was not conducted as part of these guidelines. This question's focus is the effect of using any delirium assessment tool (vs no assessment tool) in clinical practice.

Rationale: Most studies evaluating delirium assessment combine the assessment intervention with one or more management strategies $(8,110,283)$, precluding the ability to evaluate outcomes related to the monitoring itself. Three studies specifically evaluated delirium assessment effects (284-286) and varied significantly in design and choice of 
evaluated outcomes. Two $(284,285)$ found no relationship between delirium assessment and ICU LOS or duration of mechanical ventilation. Three studies evaluated time to delirium diagnosis and treatment. One study compared screening using the CAM-ICU versus clinical assessment (285) and reported no difference in time to diagnosis or treatment with antipsychotics. The CAM-ICU arm had more antipsychotic medication days, but the total dose of antipsychotic medication administered was similar in the two arms. The largest of the four studies (286) compared assessment tool implementation and haloperidol use, a proxy in that study for delirium incidence and duration. More patients in the postimplementation period were treated with haloperidol, but at lower doses and for less time than patients in the preimplementation group. In a crossover study, Reade et al (287) compared a period of CAM-ICU assessment to a period of unstructured nursing assessments using a form with a delirium definition. The CAM-ICU arm had a significantly lower proportion of nursing shifts with delirium and a shorter duration of delirium when compared with the period of unstructured assessments. Systemic delirium detection can spuriously raise reported delirium prevalence, making it challenging to capture the true impact of delirium reduction intervention efforts on this outcome. Implementation strategies differed, and each study's significant design limitations led to low and very low quality of evidence evaluations. These studies are summarized in Supplemental Table 25 (Supplemental Digital Content 33, http://links.lww.com/ CCM/D791). Although none of the studies reported patient harm, this quality level and the heterogeneity in study design and results preclude a recommendation. This evidence cannot establish whether delirium screening alone is beneficial.

Instead of a graded recommendation, we issue an ungraded Good Practice Statement given that the potential benefits of delirium monitoring far outweigh any potential downsides. Summarizing the literature and evaluating the quality of evidence was not feasible due to complexity of studies. The primary potential benefit of delirium monitoring is early recognition that may hasten clinical assessment and intervention. Early detection may lead to prompt identification and correction (when possible) of etiology, assurance of patients experiencing distressing symptoms, treatment (pharmacologic or nonpharmacologic), and treatment effectiveness assessments. Multiple studies in both ICU and non-ICU settings have found that without validated screening tools, bedside nurses and physicians fail to recognize delirium (285, 287-294).

What are the consequences of missing delirium in addition to possible earlier detection of underlying delirium causes? Delirium is a distressing experience for ICU patients, their families, and for ICU staff (295-298). Although not proven, such distress might be mitigated by discussions between staff and patients/families about delirium. Regular delirium monitoring may provide a foundation for those discussions (299). Qualitative studies of ICU experiences consistently highlight that delirious patients feel greater trust toward, and encouragement from, family members versus staff $(295,300)$. The early detection and identification of delirium might benefit patients by fostering reassurance when frightening symptoms occur.

Delirium screening using the CAM-ICU or the ICDSC is quick $(2-5 \mathrm{~min})(284,286)$. A recent systematic review has updated the psychometric properties of delirium screening tools for critically ill adults (301). The sensitivity and specificity of delirium screening tools when compared with clinical assessment, and their reproducibility and reliability when screening tools are substituted for a clinical diagnosis vary between ICU populations (e.g., cardiac surgery ICU or neurologically injured patients) $(51,302,303)$. A recent publication (304) describes a new validated tool (the ICU-7) to document delirium severity and suggests that severity is associated with worse outcome. Almost all the clinical trials investigating strategies to prevent and/or treat delirium are based on delirium assessment tools. The generalizability of any delirium-focused study relies on these instruments in clinical practice. Because the characteristics of the tools (and their confounders) are better described, the results of these investigations will help guide future clinical trials.

The disadvantages of delirium screening should be considered. A false-positive screening, although rare with either the CAM-ICU or the ICDSC, may result in unnecessary pharmacologic or nonpharmacologic treatment. ICU antipsychotic use is often associated with its continuation and prolonged administration after ICU and hospital discharge (305-307). Delirium screening may be burdensome for nursing staff (287). In the context of the criteria needed to generate a best practice statement, we felt that the benefits of widespread delirium assessment with the CAM-ICU or the ICDSC far outweigh any potential disadvantages.

Evidence Gaps: The current body of evidence in support of pain and agitation assessments, which has been studied longer than delirium, may provide some guidance for future research in delirium monitoring $(19,106,110,308-310)$. Some studies $(18,310)$ suggest that the ability of assessment tools to improve patient outcomes may be associated with the intensity of the training strategy used and the quality improvement initiatives deployed. A recent observational study (311) found an association between high delirium monitoring adherence (i.e., assessments on $\geq 50 \%$ of the ICU days) and improved patient outcomes (i.e., lower in-hospital mortality, shorter ICU LOS, and shorter time on mechanical ventilation). Future studies should include various critical care populations such as patients with primary neurologic diagnoses. The lack of high-quality trials investigating the effect of delirium assessment underscores the gaps in understanding the relationship among delirium assessment and patient-centered outcomes, treatment decisions, patient and family satisfaction, and staff satisfaction.

\section{Level of Arousal and Assessment}

Question: Does the level of arousal influence delirium assessments with a validated screening tool?

Ungraded Statement: Level of arousal may influence delirium assessments with a validated screening tool. 
Rationale: Four observational cohort studies have examined delirium assessments at different levels of wakefulness and sedation as assessed by the CAM-ICU, ICDSC, and RASS (312$315)$. Because many patients with RASS of -3 were deemed in these studies to be "unable to assess," data are limited to an evaluation of the influence of a RASS range from 0 to -2 on delirium positivity. These data do not allow for discrimination between delirium that is potentially sedation induced compared with that related to other pathologic alterations (with or without sedation).

A total of 12,699 delirium assessments (97\% involving the CAM-ICU) were evaluated in patients with a RASS between 0 and -2 . The likelihood of a positive delirium assessment was significantly greater $(77 \%$ vs $23 \%$; $p<0.0001)$ when patients had a RASS -2 (vs a RASS of -1 to 0 ), which could suggest that level of arousal influences delirium assessments. However, because delirium can present with a decreased arousal level, no inferences can be made from these data (Supplemental Table 26, Supplemental Digital Content 34, http://links.lww.com/CCM/D792). Apart from the study by Patel et al (312) in which $12 \%$ of patients in whom delirium was present during sedative infusion resolved within 2 hours of stopping infusion, no other study informs the question of whether a positive delirium assessment as a result of concomitant sedation affects patient outcome or whether sedation merely represents a confounding issue for patient assessment. Given that studies to date have shown that delirium is associated with worse outcomes, even when a depressed level of arousal is present, clinicians should not currently discount the clinical significance of delirium in this setting (316-318).

Evidence Gaps: The effects of level of arousal on delirium are in need of further study. This includes the impact of delirium at different levels of arousal on delirium assessments (with or without concomitant sedative exposure) on important outcomes such as hospital disposition and long-term cognitive impairment.

\section{Outcomes \\ Delirium.}

Questions: What are the short- and long-term outcomes of delirium in critically ill adults and are these causally related?

Ungraded Statements: Positive delirium screening in critically ill adults is strongly associated with cognitive impairment at 3 and 12 months after ICU discharge (316-319) and may be associated with a longer hospital stay (257, 279, 316, 320-327).

Delirium in critically ill adults has consistently been shown NOT to be associated with PTSD (328-333) or post-ICU distress $(316,333-336)$.

Delirium in critically ill adults has NOT been consistently shown to be associated with ICU LOS $(257,258,272,279,318$, $320-326,334,337-352)$, discharge disposition to a place other than home $(257,342,344,353,354)$, depression $(330,356)$, functionality/dependence (330, 334, 350, 353, 354, 357-360), or mortality $(316,357)$.

Rationale: Despite the fact that 48 studies enrolling 19,658 patients describe potential outcomes associated with ICU delirium, the complex relationship linking delirium to these outcomes has yet to be fully defined $(257,258,279,316-326$, 330-332, 334-354, 356-358, 360-365) (Supplemental Table 27, Supplemental Digital Content 35, http://links.lww.com/CCM/ D793). We emphasize that these associations do not imply causality and that they highlight areas for future studies particularly those involving cognition. Another significant gap in ICU delirium outcomes data includes the psychologic toll that delirium exerts in real time on patients, families, and caregivers.

\section{Rapidly Reversible Delirium.}

Question: What are the short- and long-term outcomes of rapidly reversible delirium?

Ungraded Statement: Rapidly reversible delirium is associated with outcomes that are similar to patients who never experience delirium.

Rationale: One prospective observational study with blinded evaluations enrolled 102 patients (312) and found that outcomes (ICU and hospital LOS, discharge disposition, and 1-yr mortality) were similar between the 12 patients who developed rapidly reversible, sedation-related delirium and the 10 patients who never experienced delirium. Most patients $(n=80)$ who had either delirium or not always rapidly reversible delirium had worse outcomes than the patients with rapidly reversible, sedation-related delirium, or who never developed delirium. These preliminary data suggest that for a small group of patients with rapidly reversible delirium, delirium is not associated with the specifically measured adverse clinical outcomes. Delirium assessments should be performed both before and after a DSI (SAT) to identify these subtypes of delirium.

\section{Pharmacologic Prevention and Treatment Prevention.}

Question: Should a pharmacologic agent (vs no use of this agent) be used to "prevent" delirium in all critically ill adults?

Recommendation: We suggest not using haloperidol, an atypical antipsychotic, dexmedetomidine, a $\beta$-Hydroxy $\beta$-methylglutaryl-Coenzyme A (HMG-CoA) reductase inhibitor (i.e., statin), or ketamine to prevent delirium in all critically ill adults (conditional recommendation, very low to low quality of evidence).

Rationale: The outcomes deemed critical to this recommendation included delirium incidence and duration, duration of mechanical ventilation, length of ICU stay, and mortality. Single, randomized studies of adults who were admitted to the ICU for postoperative care were reviewed for haloperidol (366); the atypical antipsychotic, risperidone (367); and dexmedetomidine (368). Each study reported a significant reduction in delirium incidence favoring the pharmacologic agent: scheduled IV haloperidol $(n=457)$ after noncardiac surgery (RR, 0.66; 95\% CI, 0.45-0.97; low quality) (366); a single dose of risperidone $(n=126)$ following elective cardiac surgery ( $R R$, 0.35; 95\% CI, 0.16-0.77; low quality) (366); and scheduled, low-dose dexmedetomidine $(n=700)$ after noncardiac surgery (odds ratio [OR], 0.35; 95\% CI, 0.22-0.54; low quality) (368). One recently published, double-blind, placebo-controlled RCT of 1,789 delirium-free critically ill adults, not included in the 
evidence profile, found that administration of low-dose IV haloperidol in the ICU until delirium developed did not help prevent delirium or affect 90-day survival (369). Another suggested that nocturnal administration of low-dose dexmedetomidine in critically ill adults with APACHE-II scores of 22 (SD, \pm 7.8 ) was associated with a significantly greater proportion of patients who remained delirium free ( $80 \%$ vs $54 \% ; p=0.008$ ) during their ICU stay (370).

Despite the consistent reduction in delirium incidence in each study, none reported a statistically significant and/or clinically meaningful difference for any of the other outcomes that the group deemed critical. The randomized trials informing this question included surgical adults having a severity of illness less than half, on average, of the (predominantly medical) ICU patients represented in these trials (366-368). Given the strong association between severity of illness and delirium occurrence (365), data derived from surgical patients with a low severity of illness must be interpreted with caution.

Many acute critically ill patients have delirium at ICU admission and thus delirium prevention strategies may not apply to this proportion of the ICU population. Given this evidence gap and the lack of generalizability from each study population to the broader critically ill adult population, the current recommendation reflects the panel's concern that the potential risks and costs of exposing a large proportion of the critically ill adult population to one or more medications aimed at preventing delirium will outweigh any benefit.

Three cohort studies suggest that when statin use is stopped during critical illness, delirium occurrence increases (371-373). However, one recent randomized study of delirium-free cardiac surgery patients admitted to the ICU (not included in the evidence profile for this question) found that the use of preoperative atorvastatin did not affect incident delirium (374). The role of an NMDA receptor antagonist for the primary prevention of delirium prevention in critically ill adults was being prospectively evaluated in a randomized trial at the time of guideline development. One recent large RCT found that a single subanesthetic dose of ketamine, administered perioperatively, did not decrease delirium in older adults after major surgery, some of who required admission to the ICU (375).

\section{Subsyndromal Delirium Treatment.}

Question: Should a pharmacologic agent (vs no use of this agent) be used to "treat subsyndromal delirium" in all critically ill adults with subsyndromal delirium?

Recommendation: We suggest not using haloperidol or an atypical antipsychotic to treat subsyndromal delirium in critically ill adults (conditional recommendations, very low to low quality of evidence).

Rationale: Subsyndromal delirium is part of an outcomepredicting spectrum of delirium symptoms, is present when the ICDSC score is $1-3$ out of 8 and occurs in about $30 \%$ of critically ill adults (342). A critically ill patient who develops subsyndromal delirium, compared with one who develops neither delirium (ICDSC, $\geq 4$ ) nor subsyndromal delirium, is more likely to die in the ICU, spend more time hospitalized, and to be discharged to a long-term care facility rather than home (342). Duration of subsyndromal delirium when evaluated using the CAM-ICU is an independent predictor of increased odds of institutionalization (376). The outcomes deemed critical to this recommendation included delirium incidence, duration, and severity; duration of mechanical ventilation; ICU LOS; and mortality. Both RCTs used the ICDSC to identify patients with subsyndromal and full-syndrome delirium (ICDSC, $\geq 4$ ). Scheduled IV haloperidol $1 \mathrm{mg}$ q6h, when compared with placebo in 60 mechanically ventilated adults, was not associated with a change in delirium incidence, duration, or time to first episode of delirium; days of mechanical ventilation; or ICU LOS in critically ill medical and surgical patients (377). Risperidone $(0.5 \mathrm{mg}$ every $8 \mathrm{hr})$, when compared with placebo in 101 cardiac surgery patients, was associated with a reduced likelihood for a transition from subsyndromal to fullsyndrome delirium (RR, 0.41; 95\% CI, 0.02-0.86) (378).

Despite this reduction in delirium incidence, neither statistically significant and/or clinically meaningful differences were noted for any of the other outcomes deemed critical by the group. Given these evidence gaps, questionable clinical benefit, and the potential lack of applicability of data from the study by Hakim et al (378) to the entire medical and surgical critically ill population having a greater severity of illness and different risk factors for delirium, the current recommendation reflects the panel's concern about the risks of exposing up to $35 \%$ of all critically ill adults to antipsychotic therapy (379). The role of dexmedetomidine, a HMG-CoA reductase inhibitor (i.e., a statin), or an NMDA antagonist (e.g., ketamine) as a treatment for subsyndromal delirium has not been evaluated in a randomized trial.

\section{Delirium Treatment.}

Question: Should a pharmacologic agent (vs no use of this agent) be used to treat delirium in all critically ill adults with delirium?

\section{Antipsychotic/statin.}

Recommendation: We suggest not routinely using haloperidol, an atypical antipsychotic, or a HMG-CoA reductase inhibitor (i.e., a statin) to treat delirium (conditional recommendation, low quality of evidence).

Rationale: The outcomes deemed most critical to this question included delirium duration, duration of mechanical ventilation, ICU LOS, and mortality. A total of six RCTs were identified: haloperidol $(n=2)(380,381)$, atypical antipsychotics (quetiapine) $(n=1)(382)$, ziprasidone $(n=1)(380)$, olanzapine $(n=1)$ (383), and a statin (i.e., rosuvastatin) $(n=$ 1) (384). A recent randomized trial of critically ill adults, not included in the evidence profile, found that high-dose simvastatin does not reduce days spent with delirium and coma (385). No evidence was found to inform a recommendation regarding the use of an NMDA antagonist (e.g., ketamine) for delirium treatment.

This evidence suggests that the use of the typical antipsychotic, haloperidol; an atypical antipsychotic (e.g., quetiapine, ziprasidone); or a statin was not associated with a shorter duration of delirium, a reduced duration of mechanical ventilation 
or ICU LOS, or decreased mortality. Although the randomized trials informing this question were conducted in both medical and surgical patients who were critically ill, each used open-label antipsychotic rescue medication for agitation or hallucinations $(368,380-384,386)$. Administration of such open-label medication to the placebo group in these studies may bias the results of these investigations toward the null hypothesis. The undesirable effects of haloperidol and atypical antipsychotics remain uncertain, given the small sample sizes of the available studies.

Although this recommendation discourages the "routine" use of antipsychotic agents in the treatment of delirium, patients who experience significant distress secondary to symptoms of a delirium such as anxiety, fearfulness, hallucinations, or delusions, or who are agitated and may be physically harmful to themselves or others, may benefit from short-term use of haloperidol or an atypical antipsychotic until these distressing symptoms resolve based on the panel's clinical experience. Patients who start with an antipsychotic for delirium in the ICU often remain on these medications unnecessarily after discharge (305-307). Continued exposure to antipsychotic medication can result in significant morbidity and financial cost. Panel members judged that the undesirable consequences of using either haloperidol or an atypical antipsychotic far outweighed the potential benefits for most critically adults with delirium and thus issued a conditional recommendation against their routine use.

\section{Dexmedetomidine.}

Recommendation: We suggest using dexmedetomidine for delirium in mechanically ventilated adults where agitation is precluding weaning/extubation (conditional recommendation, low quality of evidence).

Rationale: The single RCT used to evaluate the role of dexmedetomidine as a treatment for agitation precluding ventilator liberation in patients with delirium screened 21,500 intubated patients from 15 ICUs to enroll the 71 study patients and was terminated early because the funding amount (from the manufacturer of dexmedetomidine) had been used up (386). Although dexmedetomidine (vs placebo) was associated with a small, but statistically significant increase in ventilator-free hours in the first 7 days after study randomization (MD, $17.3 \mathrm{hr}$; $95 \%$ CI, 4.0 33.2 ; very low quality), its use did not affect either ICU or hospital LOS, or patient's disposition location at hospital discharge. Patients did not commonly receive opioids; some of the agitation may have been pain related; and the number of patients enrolled with acute alcohol withdrawal was not reported.

Panel members judged that the desirable consequences of using dexmedetomidine for mechanically ventilated ICU patients with agitation precluding weaning/extubation outweighed the potential undesirable consequences associated with its use; therefore, they issued a conditional recommendation supporting its use in the narrow population of critically ill adults. The role of dexmedetomidine in patients with delirium without agitation or who have agitation that is not precluding ventilator liberation remains unclear. Recommendations regarding choice of sedation in mechanically ventilated critically ill adults in the context of delirium can be found in recommendations about sedative choice.
Evidence Gaps: Studies evaluating pharmacologic prevention strategies need to evaluate patients without delirium, enroll severely ill medical patients, identify patient subgroups where the delirium prevention benefits are greatest, and evaluate clinically meaningful outcomes. To improve the methodology of such subsyndromal treatment trials, our understanding of the significance, characteristics, and measurement of subsyndromal delirium needs to expand. In addition, future studies should target specific symptoms (e.g., anxiety) instead of subsyndromal delirium as a whole. Delirium treatment studies should focus on more homogeneous high-risk ICU populations given that the cause of delirium (and thus response to therapy) may be different. Symptomatic distress (e.g., agitation) and long-term cognitive and functional outcome should be evaluated. Medications shown in small studies to reduce delirium symptoms (e.g., valproic acid) should be rigorously evaluated. Finally, system innovations are needed to ensure that patients do not remain indefinitely on medications such as antipsychotics after symptomatic initiation during an ICU episode of delirium.

\section{Nonpharmacologic Prevention and Treatment Single Component.}

Question: Should a single-component, nonpharmacologic strategy not solely focused on sleep improvement or early mobilization (vs no such strategy) be used to reduce delirium in critically ill adults?

Recommendation: We suggest not using bright light therapy to reduce delirium in critically ill adults (conditional recommendation, moderate quality of evidence).

Rationale: ICU delirium studies of nonpharmacologic interventions focused on either one modifiable risk factor with a single intervention or several modifiable risk factors with multicomponent interventions (Supplemental Table 28, Supplemental Digital Content 36, http://links.lww.com/ CCM/D794). For the purposes of these guidelines, one question addressed single intervention studies and one question addressed multicomponent intervention studies. Delirium incidence, prevalence, and duration were considered the most important outcomes across both questions. ICU LOS, hospital LOS, and hospital mortality were also considered to be critical outcomes for these questions. Bright light therapy, family participation in care, and a psychoeducational program were the only single-component interventions that have been studied in the ICU.

Three studies examined the effects of light therapy, which did not demonstrate beneficial effect on either delirium incidence or ICU LOS (387-389). One before-after study evaluated the effect of family participation in care (390). Panel members judged that the undesirable consequences of using bright light therapy outweighed the potential desirable effects associated with its use and thus issued a conditional recommendation against its use.

\section{Multicomponent.}

Question: Should a multicomponent, nonpharmacologic strategy (vs no such strategy) be used to reduce delirium in critically ill adults? 
Recommendation: We suggest using a multicomponent, nonpharmacologic intervention that is focused on (but not limited to) reducing modifiable risk factors for delirium, improving cognition, and optimizing sleep, mobility, hearing, and vision in critically ill adults (conditional recommendation, low quality of evidence).

Remarks: These multicomponent interventions include (but are not limited to) strategies to reduce or shorten delirium (e.g., reorientation, cognitive stimulation, use of clocks); improve sleep (e.g., minimizing light and noise); improve wakefulness (i.e., reduced sedation); reduce immobility (e.g., early rehabilitation/mobilization); and reduce hearing and/or visual impairment (e.g., enable use of devices such as hearing aids or eye glasses).

Rationale: The multicomponent intervention studies evaluated a bundle of interventions. Many examples of multicomponent bundles $(8,283,391-396)$ have shown improved outcomes in critically ill adults (Supplemental Table 29, Supplemental Digital Content 37, http://links.lww.com/CCM/ D795). Pilot studies suggested that combining cognitive and physical therapy early during critical illness is feasible and safe (391) and using nonpharmacologic multicomponent interventions in ICU patients is feasible (392). Studies of multicomponent interventions, many of which were not randomized, focus on cognitive impairment (e.g., reorientation, cognitive stimulation, music, use of clocks); sedation/sleep disruption (e.g., reducing sedation, minimizing light and noise); immobility (early rehabilitation/mobilization); and hearing and visual impairment (e.g., use of hearing aids and glasses). Overall, the use of such strategies reduced delirium significantly (five studies, $n=1,318$; OR, 0.59; 95\% CI, 0.39-0.88) (392-396). Further, ICU duration of delirium (16 vs $20 \mathrm{hr}$ ) (395), ICU LOS (387), and hospital mortality all decreased (393).

Another multi-intervention approach, the awakening and breathing coordination, delirium monitoring/management, and early exercise/mobility (ABCDE) bundle, was significantly associated with less delirium ( $n=296 ; 49 \%$ vs $62 \%$; OR, 0.55 ; 95\% CI, 0.33-0.93) (7) when evaluated in a before-after study at one hospital. When a revised and expanded ABCDEF bundle (which includes a focus on "F," Family engagement) was evaluated in a larger, multicenter, before-after, cohort study, and where delirium was also assessed using the CAM-ICU, an adjusted analysis showed that improvements in bundle compliance were significantly associated with reduced mortality and more ICU days without coma or delirium (9). Adverse effects were not reported in these nonpharmacologic intervention studies. Six of the eight studies' small interventions were heterogeneous, and the studies with positive findings were observational. Panel members judged that desirable consequences of using any of these multicomponent interventions to reduce delirium outweighed any potential undesirable consequences and thus issued a conditional recommendation supporting their use.

Evidence Gaps: Overall, the certainty of evidence supporting single-component and multicomponent interventions is low. Because delirium almost always has a multifactorial etiology, multicomponent interventions are plausibly more promising than single interventions. However, a major gap in understanding the available data is uncertainty as to which interventions result in the effect. The role of families in reducing patient stress and facilitating nonpharmacologic delirium prevention and management interventions requires further research. The experience of patients with delirium has not been qualitatively evaluated. Some articles describe the same interventions differently (2); consistent definitions should be established.

\section{IMMOBILITY (REHABILITATION/ MOBILIZATION)}

Survivors of critical illness frequently experience many longterm sequelae, including ICU-acquired muscle weakness (ICUAW). ICUAW can be present in $25-50 \%$ of critically ill patients (397) and is associated with impairments in patients' long-term survival, physical functioning, and quality of life (398-400). One important risk factor for ICUAW is bed rest $(398,401)$. The safety, feasibility, and benefits of rehabilitation and mobilization delivered in the ICU setting have been evaluated as potential means to mitigate ICUAW and impaired physical functioning.

As highlighted in the 2013 guidelines (1), rehabilitation/ mobilization may be beneficial as part of delirium management strategies. Furthermore, important associations exist between analgesic and sedation practices and pain and sedation status with patients' participation in rehabilitation/mobilization in the ICU (402). Given the growing literature in this field and the interplay of rehabilitation/mobilization with pain, agitation, and delirium, this topic was introduced as a new part of the present guideline. One actionable question and three descriptive questions were addressed (see prioritized topic list in Supplemental Table 30 [Supplemental Digital Content 38, http://links.lww.com/CCM/ D796] and voting results in Supplemental Table 31 [Supplemental Digital Content 39, http://links.lww.com/CCM/D797]) (403). A glossary of rehabilitation /mobilization interventions and outcomes relevant to this topic can be found in Supplemental Table 32 (Supplemental Digital Content 40, http://links.lww.com/CCM/ D798). The evidence summaries and evidence-to-decision tables used to develop recommendations for the immobility (rehabilitation/mobilization) group are available in Supplemental Table 33 (Supplemental Digital Content 41, http://links.lww.com/CCM/ D799), and the forest plots for all meta-analyses are available in Supplemental Figure 8 (Supplemental Digital Content 42, http:// links.lww.com/CCM/D800).

\section{Efficacy and Benefit}

Question: For critically ill adults, is receiving rehabilitation or mobilization (performed either in-bed or out-of-bed) beneficial in improving patient, family, or health system outcomes compared with usual care, a different rehabilitation/mobilization intervention, placebo, or sham intervention?

Recommendation: We suggest performing rehabilitation or mobilization in critically ill adults (conditional recommendation, low quality evidence). 
Remarks: Rehabilitation is a "set of interventions designed to optimize functioning and reduce disability in individuals with a health condition" (404). Mobilization is a type of intervention within rehabilitation that facilitates the movement of patients and expends energy with a goal of improving patient outcomes (405). This recommendation supports performing rehabilitation/mobilization interventions over usual care or over similar interventions with a reduced duration, reduced frequency, or later onset. The implementation of this recommendation will be influenced by feasibility-related issues, particularly related to variability in the availability of appropriate staffing and resources to perform rehabilitation/mobilization interventions across ICUs.

Rationale: A wide variety of critically ill patient populations were studied (see study eligibility criteria in Supplemental Table 34 [Supplemental Digital Content 43, http://links.lww. $\mathrm{com} / \mathrm{CCM} / \mathrm{D} 801])$. Studies evaluated different types of interventions and different timings for initiating the intervention, which prevent us from making more specific recommendations in these areas. Comparators for the interventions included usual care rehabilitation or mobilization; rehabilitation or mobilization interventions with reduced duration or frequency; or a longer time to initiation compared with the intervention group. As described below, five outcomes were evaluated for this question. Three additional outcomes (cognitive function, mental health, and timing of return to work and related economic outcomes) could not be evaluated due to inadequate data.

We identified a total of 16 RCTs (391, 406-420) (Supplemental Table 25, Supplemental Digital Content 33, http://links.lww.com/CCM/D791) that met our eligibility criteria and reported on five critical outcomes. The pooled estimates from six RCTs (304 patients) showed that rehabilitation/mobilization improved muscle strength at ICU discharge (MD in Medical Research Council sum score [range, 0-60]: 6.24 points [95\% CI, 1.67-10.82; low quality evidence]) (408-410, 414, 415,420 ). Duration of mechanical ventilation (11 RCTs, 1,128 patients) was reduced by 1.31 days ( $95 \%$ CI, -2.44 to -0.19 ; low quality evidence) (406-409, 411, 413-416). For health-related quality of life measured using the 36-Item Short Form Health Survey instrument within 2 months of discharge in four RCTs (303 patients), a moderate-sized improvement (SMD, 0.64 [ $95 \% \mathrm{CI},-0.05$ to 1.34$]$ ) not reaching statistical significance was observed, with an overall rating of low quality of evidence (412, 416-418). For the remaining two critical outcomes, across 13 RCTs ( 1,421 patients), there was no effect on hospital mortality (moderate quality of evidence) (391, 407, 408, 410-418, 420). Physical function was evaluated via the "Timed Up and Go" test in three RCTs (209 patients) and the Physical Function in ICU Test in three RCTs (209 patients), with no significant effect of rehabilitation/mobilization (moderate quality of evidence) (391, 411, 414, 416, 420). The incidence of adverse events for patients was very low based on five trials and eight observational studies (moderate quality of evidence).

Rehabilitation/mobilization was assessed as feasible, acceptable to key stakeholders, and likely to be cost-effective based on preliminary data. In addition, indirect evidence (421), along with a discussion with panel members (including an ICU patient representative), suggests that patients will probably value the benefits of rehabilitation/mobilization. Given a small benefit of rehabilitation/mobilization interventions (performed either in-bed or out-of-bed) and the low overall quality of evidence, panel members agreed that the desirable consequences for patients probably outweigh the undesirable consequences, and issued a conditional recommendation favoring rehabilitation/mobilization interventions.

\section{Safety and Risk}

Question: For critically ill adults, is receiving rehabilitation/ mobilization (performed either in-bed or out-of-bed) commonly associated with patient-related safety events or harm?

Ungraded Statement: Serious safety events or harms do not occur commonly during physical rehabilitation or mobilization.

Rationale: Data from 10 observational and nine RCTs (Supplemental Table 35, Supplemental Digital Content 44, http://links.lww.com/CCM/D802) were reviewed to answer this question. Serious safety events or harms were defined as a change in physiologic status or an injury that required an intervention. These events were rare, with only 15 reported during greater than 12,200 sessions across 13 studies $(283,391$, $416-418,422-429)$. An incidence rate for these events could not be calculated because information about the number of patients at risk and/or the number of rehabilitation/mobilization sessions per patient was not consistently or clearly reported in many studies.

The majority of safety events or harms was respiratory related, with four desaturations that required an increase in $\mathrm{FiO}_{2}$ $(423,429)$ and three unplanned extubations (285). Three musculoskeletal-related events occurred: one fall (427), one Achilles tendon rupture (418), and one polyarthralgia exacerbation (416). Two cardiovascular-related events occurred: one hypertensive urgency (391) and one syncopal episode (416). Overall, patient harm related to rehabilitation/mobilization is rare; this conclusion is supported by a recent meta-analysis (430).

\section{Indicators for Initiation}

Question: For critically ill adults, what aspects of patient clinical status are indicators for the safe initiation of rehabilitation/ mobilization (performed either in-bed or out-of-bed)?

Ungraded Statements: Major indicators for safely initiating rehabilitation/mobilization include stability in cardiovascular, respiratory, and neurologic status.

Vasoactive infusions or mechanical ventilation are not barriers to initiating rehabilitation/mobilization, assuming patients are otherwise stable with the use of these therapies.

Rationale: Safe initiation of physical rehabilitation or mobilization was evaluated in 17 (283, 391, 407, 408, 413, 416-418, 424-426, 429, 431-435) studies that enrolled 2,774 patients and reported cardiovascular, respiratory, or neurologic criteria (Supplemental Table 36, Supplemental Digital Content 45, http://links.lww.com/CCM/D803). Data from these studies 
were summarized, and expert opinion was used to determine suggested ranges for cardiovascular, respiratory, neurologic, and other relevant criteria within which rehabilitation/mobilization can be safely initiated (Table 1). Although these parameters were based on clinical research with clinical application interpreted via expert opinion, they should not be a substitute for clinical judgment. All thresholds should be interpreted or modified, as needed, in the context of individual patients' clinical symptoms, expected values, recent trends, and any clinician-prescribed goals or targets.

\section{Indicators for Stopping}

Question: For adult critically ill patients, what aspects of patient clinical status are indicators that rehabilitation/mobilization (performed either in-bed or out-of-bed) should be stopped?

Ungraded Statements: Major indicators for stopping rehabilitation/mobilization include development of new cardiovascular, respiratory, or neurologic instability.

Other events, such as a fall or medical device removal/malfunction, and patient distress are also indications for stopping.

Rationale: Indicators for stopping rehabilitation/mobilization were reported in 14 studies $(283,391,407,408,413$, $416,418,424,425,429,431-434)$ that enrolled 2,617 patients (Supplemental Table 37, Supplemental Digital Content 46, http://links.lww.com/CCM/D804). Specific stopping criteria for cardiovascular, respiratory, or neurologic instability were identified. Data from these studies were summarized, and expert opinion was used to determine suggested cardiovascular, respiratory, neurologic, and other relevant criteria for stopping rehabilitation/mobilization (Table 1). Although these parameters were based on clinical research with clinical application interpreted via expert opinion, they should not be a substitute for clinical judgment.

Evidence Gaps: The field of ICU-based rehabilitation/ mobilization is at an early stage with a rapidly evolving body of evidence. Many research questions remain outstanding. Important directions for future research include understanding differences in patient outcomes according to the type of intervention and the timing, frequency, duration, and intensity of interventions. The mode of intervention delivery, including the expertise/training of personnel delivering interventions, needs additional investigation. The influence of patient conditions (e.g., pre-ICU functional status, delirium and sedation status, muscle wasting, and nerve and muscle dysfunction) on patient outcomes after rehabilitation/mobilization interventions should be examined. These factors may help to identify potential subgroups of critically ill patients who may gain the greatest benefit from rehabilitation/mobilization interventions. As well, methods to assess the patient experience during rehabilitation/mobilization, particularly in nonverbal critically ill patients, are warranted. Standardized reporting of intervention details (e.g., timing, frequency, duration, and intensity), potential safety events, and both short-term and long-term outcomes will facilitate comparisons between studies and settings. Finally, future research should continue to evaluate the measurement properties of short-term and long-term outcome measures to determine the most effective and efficient approaches to evaluating the effects of rehabilitation/ mobilization.

\section{SLEEP DISRUPTION}

Poor sleep is a common complaint and a source of distress for many critically ill patients $(436,437)$. Sleep disruption in the critically ill can be severe and is characterized by sleep fragmentation, abnormal circadian rhythms, increased light sleep (stage $\mathrm{N} 1+\mathrm{N} 2$ ), and decreased slow-wave (stage N3) and rapid eye movement (REM) sleep (438-440). The interplay of medications, critical illness, delirium, cerebral perfusion, and sleep is complex, but is important, and is an increasing focus of research. A glossary of the sleep-related terms used in this section can be found in Supplemental Table 38 (Supplemental Digital Content 47, http://links.lww.com/CCM/D805), and an overview of normal sleep and its architecture as characterized by polysomnography can be found in Supplemental Table 39 (Supplemental Digital Content 48, http://links.lww.com/CCM/D806).

In addition to emotional distress, sleep disruption has also been hypothesized to contribute to ICU delirium (441-443), prolonged duration of mechanical ventilation (444), deranged immune function $(445,446)$, and neurocognitive dysfunction. Given that sleep is a potentially modifiable risk factor influencing recovery in critically ill adults, this topic has been introduced in the present guideline and is addressed in four actionable and six descriptive questions (see prioritized topic list in Supplemental Table 40 [Supplemental Digital Content 49, http://links.lww.com/CCM/D807] and voting results in Supplemental Table 41 [Supplemental Digital Content 50, http://links.lww.com/CCM/D808]). The evidence summaries and evidence-to-decision tables used to develop recommendations for the disrupted sleep group are available in Supplemental Table 42 (Supplemental Digital Content 51, http://links.lww.com/CCM/D809), and the forest plots for all meta-analyses completed are available in Supplemental Figure 9 (Supplemental Digital Content 52, http://links.lww. com/CCM/D810).

\section{Characterization}

\section{Critically Ill Versus Healthy.}

Question: How does sleep in critically ill adults differ from normal sleep in healthy adults?

Ungraded Statements: Total sleep time (TST) and sleep efficiency are often normal.

Sleep fragmentation, the proportion of time spent in light sleep (stages N1 + N2), and time spent sleeping during the day (vs night) are higher.

The proportion of time spent in deep sleep (stage N3 sleep and REM) is lower.

Subjective sleep quality is reduced.

Rationale: Small studies suggest that TST and sleep efficiency are normal during critical illness although considerable interpatient variability exists $(443,447)$. During critical illness, the proportion of time spent in light sleep (stages $\mathrm{N} 1+\mathrm{N} 2$ ) is increased and the time spent in deep sleep (stages N3 + REM 


\section{TABLE 1. Summary of Safety Criteria for Starting and Stopping Physical Rehabilitation or Mobilization Performed Either In-Bed or Out-of-Bed}

\begin{tabular}{|c|c|c|}
\hline System & Starting a Rehabilitation/Mobility Session ${ }^{\mathrm{a}}$ & Stopping a Rehabilitation/Mobility Session ${ }^{a}$ \\
\hline Cardiovascular & $\begin{array}{l}\text { - Heart rate is between } 60 \text { and } 130 \text { beats/min, } \\
\text { - Systolic blood pressure is between } 90 \text { and } \\
180 \mathrm{~mm} \mathrm{Hg} \text {, or } \\
\text { - Mean arterial pressure is between } 60 \text { and } 100 \mathrm{~mm} \mathrm{Hg}\end{array}$ & $\begin{array}{l}\text { - Heart rate decreases below } 60 \text { or increases } \\
\text { above } 130 \text { beats/min, } \\
\text { - Systolic blood pressure decreases below } 90 \text { or } \\
\text { increases above } 180 \mathrm{~mm} \mathrm{Hg} \text {, or } \\
\text { - Mean arterial pressure decreases below } 60 \text { or } \\
\text { increases above } 100 \mathrm{~mm} \mathrm{Hg}\end{array}$ \\
\hline Respiratory & $\begin{array}{l}\text { - Respiratory rate is between } 5 \text { and } 40 \text { breaths/min } \\
\text { - } \mathrm{SpO}_{2} \geq 88 \% \\
\text { - } \mathrm{FlO}_{2}<0.6 \text { and positive end-expiratory pressure }<10 \\
\text { - Airway (endotracheal tube or tracheostomy) is adequately } \\
\text { secured }\end{array}$ & $\begin{array}{l}\text { - Respiratory rate decreases below } 5 \text { or increases } \\
\text { above } 40 \text { breaths per minute } \\
\text { - } \mathrm{Spo}_{2} \text { decreases below } 88 \% \\
\text { - Concerns regarding adequate securement of } \\
\text { airway (endotracheal tube or tracheostomy) }\end{array}$ \\
\hline \multirow{2}{*}{ Neurologic } & $\begin{array}{l}\text { Further, the following clinical signs and symptoms should } \\
\text { be "absent": }\end{array}$ & $\begin{array}{l}\text { Further, if the following clinical signs, symptoms or } \\
\text { events develop and appear clinically relevant: }\end{array}$ \\
\hline & $\begin{array}{l}\text { - New or symptomatic arrhythmia } \\
\text { - Chest pain with concern for myocardial ischemia } \\
\text { - Unstable spinal injury or lesion } \\
\text { - Unstable fracture } \\
\text { - Active or uncontrolled gastrointestinal bleed }\end{array}$ & $\begin{array}{l}\text { - New/symptomatic arrhythmia } \\
\text { - Chest pain with concern for myocardial ischemia } \\
\text { - Ventilator asynchrony } \\
\text { - Fall } \\
\text { - Bleeding } \\
\text { - Medical device removal or malfunction } \\
\text { - Distress reported by patient or observed by clini- } \\
\text { cian }\end{array}$ \\
\hline
\end{tabular}

$\mathrm{Spo}_{2}=$ oxygen saturation.

aBased on published clinical studies and expert opinion, but should not be a substitute for clinical judgment. All thresholds should be interpreted or modified, as needed, in the context of individual patients' clinical symptoms, "normal" values, and recent trends while in the hospital, and any clinician-prescribed goals or targets.

sleep) is decreased $(438,440,448-450)$. Sleep fragmentation (i.e., number of arousals and awakenings per hour) is higher in critically ill adults than healthy subjects $(449,451,452)$. Among healthy adults exposed to the ICU environment, daytime sleep was found to increase each day spent in the ICU setting and represent one third of total sleep (453). In critically ill adults, the proportion of total sleep during the daytime sleep has been shown to be as high as $57 \%(444,454)$. Subjective sleep quality is severely altered during critical illness; patients assess their sleep quality in the ICU as being considerably worse than their sleep at home $(449,455,456)$.

\section{Delirium Versus No Delirium.}

Question: Is sleep different in critically ill adults if delirium (vs no delirium) is present?

Ungraded Statements: The presence of delirium may not affect TST, sleep efficiency, or sleep fragmentation.
The influence of delirium on the proportion of time spent in light $(\mathrm{N} 1+\mathrm{N} 2)$ versus deeper (N3) sleep is unknown.

REM sleep is lower if delirium is present.

Delirium is associated with greater circadian sleep-cycle disruption and increased daytime sleep.

Whether delirium affects reported subjective sleep quality remains unclear.

Rationale: Delirium has not been evaluated in most ICU polysomnography sleep studies. Four studies have evaluated sleep with polysomnography in critically ill adults with delirium that was evaluated with a validated screening tool $(443,447,457)$. Two of the studies excluded patients receiving sedation $(443,447)$. TST and sleep efficiency are similar between delirious and nondelirious patients $(443,447)$. One small study of noninvasive ventilation (NIV) patients found that sleep fragmentation is similar regardless of delirium status (443). The influence of delirium on the proportion 
of time spent in light sleep (N1 + N2) (vs deeper N3 sleep) was not reported in any of the studies. The amount of REM sleep was significantly lower in patients with delirium (443). Days with delirium were greater in those patients having a very low amount of REM sleep suggesting that an association between REM sleep quantity and delirium exists (442). One study found that delirium is associated with a greater circadian sleep-cycle disruption as evidenced by daytime sleep becoming a greater proportion of TST (443). Higher reported subjective sleep quality was associated with lower delirium incidence in one observational study (312), and in one RCT, earplug use reduced delirium and improved subjective sleep quality (458). In a pre-post sleep quality improvement study, patients rated their sleep before and during the multicomponent sleep protocol similarly although significantly fewer patients had coma/ delirium during the intervention (459). Subjective sleep quality reporting by delirious patients might be unreliable.

\section{Mechanical Ventilation Versus No Mechanical Ventilation.}

Question: Is sleep different in critically ill adults who are mechanically ventilated (vs not mechanically ventilated)?

Ungraded Statements: The use of mechanical ventilation in critically ill adults may worsen sleep fragmentation, architecture, and circadian rhythm (daytime sleep) compared with normal sleep, but these effects are often variable and have not yet been fully investigated.

The use of mechanical ventilation (vs periods without mechanical ventilation) in patients with respiratory failure may improve sleep efficiency and reduce fragmentation, but data are limited.

Rationale: Ventilation and sleep share complex and reciprocal relationships. During sleep, oxygen consumption and $\mathrm{CO}_{2}$ production decrease, leading to a physiologic reduction of ventilation compared with wakefulness. Excessive pressure support, ventilator asynchronies, or ventilator alarms might trigger arousals and sleep interruptions. For the purpose of this question, "ventilated" referred to patients mechanically ventilated (both invasively and noninvasively) and "nonventilated" as patients who were breathing without any respiratory assistance (i.e., no pressure support, patients may be receiving continuous positive airway pressure). Only studies that incorporated polysomnography assessment were evaluated.

Although three polysomnography studies compared distinct ventilated and nonventilated groups $(451,454,461)$, two studies evaluated the same patients before and after ventilatory assistance $(451,454)$. During ventilation, sleep duration has been reported to be lower than normal $(241,443,448,453$, $454,462-464)$, normal $(438,465)$, or higher than normal $(466$, 467). Arousal indices are lower during ventilation (460), and sleep fragmentation is lower with NIV than without ventilation $(243,443,448,453,454,462-464)$.

Sleep fragmentation is higher during mechanical ventilation (vs no ventilation) (449) and NIV (vs no ventilation) (454). The proportion of time spent in stage N3 sleep is decreased in ventilated critically ill adults $(0-27 \%)$ (438, $439,448,449,453,462,464,467-473)$, as is the proportion of time spent in REM stage sleep reduced (0-14\%) (241, $438,440,443,448,450,451,453,454,462,464-473)$. Sleep fragmentation index during mechanical ventilation ranges from 18 to 35 arousals and awakenings per hour of sleep (241, 438, 440, 443, 448, 450, 451, 453, 454, 462, 464-474). Respiratory-related arousals have been suspected to be a major factor involved in sleep fragmentation in critically ill adults, reported in one study as causing 19\% (11-30) of arousal and awakenings from sleep (241, 438, 440, 448, 450, $451,453,454,460,462,464-474)$. Among ventilated critically ill adults, studies consistently show that among ventilated patients, the proportion of time spent in daytime sleep ranges from $36 \%$ to $57 \%$ and is greater than it is for nonventilated patients $(438,440,443,450,453,454,470)$.

Comparing mechanical ventilation (vs no mechanical ventilation) in critically ill adults, three studies have shown greater TST during mechanical ventilation $(241,436,438)$, whereas one study showed no difference (461). In patients with a tracheostomy, median (interquartile range) sleep efficiency is higher during ventilation (61\% [38-74]) than without ventilatory support (44\% [9-63]) (451). Two studies have shown that sleep fragmentation is significantly lower during mechanical ventilation (vs no ventilation) $(472,473)$, whereas one study showed no difference (451). Two studies showed no significant difference in sleep stages, whereas one study showed improved sleep architecture with less light, sleep (stage N1), and more deep sleep (stages 3 and REM sleep) during periods with NIV than without (454).

Evidence Gaps: Large, additional studies are required to define the influence of critical illness, delirium, and mechanical ventilation on sleep quality. A systematic assessment of delirium should be done in parallel with polysomnography recording (472). Among studies, considerable variability has been reported regarding all sleep parameters. These discordances might be due to several factors such as total recording time, quality of the recordings, experience of the scorer (awareness of atypical sleep), the criteria used to analyze sleep (i.e., Rechtschaffen et Kales vs Drouot-Watson rules) (457, $475,476)$, disease severity, LOS on the day of polysomnography evaluation, both sedative type and depth of sedation, and whether delirium is present. Harmonization in scoring rules and recording practices (e.g., systematic recording of noise levels and mental status) and studying homogeneous groups of patients might help to assess the prevalence of sleep alterations in critically ill patients. Detailed data on potential sleep disrupters are important when evaluating sleep fragmentation. The effect of sleep disruption on clinically relevant short- and long-term outcomes in large homogeneous patient groups remains uncertain. Finally, reliable tools to assess circadian rhythm disruption have yet to be identified.

\section{Prevalence of Unusual/Dissociated Sleep}

Question: What is the prevalence of unusual or dissociative sleep patterns in critically ill adults?

Ungraded Statement: The prevalence of unusual or dissociated sleep patterns is highly variable and depends on patient characteristics. 
Rationale: Atypical sleep, characterized by $\delta$ waves without any cyclic organization and by the absence of $\mathrm{K}$ complexes and sleep spindles that are considered the defining electroencephalogram features of stage N2 sleep, was first reported in sedated patients (438). Pathologic wakefulness is often associated with atypical sleep and is characterized by a nonreactive slowed electroencephalogram and by dissociation between electroencephalogram rhythms and behavioral wakefulness. During atypical sleep, not included in conventional Rechtschaffen and Kales electroencephalogram scoring rules, the electroencephalogram can display $\delta$ or $\theta$ waves (evocative of sleep) in behaviorally awake patients or $\alpha-\beta$ waves (evocative of wakefulness) in a comatose patient $(457,476)$.

Eleven studies have reported the prevalence of the abnormal sleep electroencephalogram patterns that meet the criteria for atypical sleep $(438,440,443,450,457,464,476-481)$. In conscious nonsedated or lightly sedated ICU patients, the abnormal sleep electroencephalogram pattern prevalence ranges from $23 \%$ to $31 \%(440,443,450,457,480)$. When criteria are used to exclude patients with known factors for these abnormal electroencephalogram patterns (e.g., receiving sedative/opioids, having delirium coma or sepsis, or with a history of epilepsy), the prevalence of atypical sleep becomes nonexistent (0\%) (464). In sedated patients, the prevalence of at least one dissociated electroencephalogram pattern (dissociated wake or sleep) ranges from $60 \%$ to $97 \%(438,476$, 481 ), and the prevalence of isolated unusual sleep electroencephalogram patterns ranges from $50 \%$ to $70 \%(475,481)$. Variability in the presence to those factors (i.e., sedation, sepsis, and delirium) known to influence abnormal sleep electroencephalogram patterns likely accounts for the variability in prevalence among studies $(438,440,457,476)$.

Evidence Gaps: Sleep recordings in critically ill adults should be carefully examined to identify new unusual or dissociative sleep patterns using published approaches and specific criteria (457, 476). The clinical characteristics of patients with these unusual patterns, and their associated mechanisms and outcomes both during and long after the ICU stay, should be investigated.

\section{Risk Factors}

\section{Before ICU Admission.}

Question: What risk factors that exist before the onset of critical illness affect sleep quality in critically ill adults in the ICU?

Ungraded Statement: Patients who report poor-quality sleep and/or use of a pharmacologic sleep aid at home are more likely to report poor-quality sleep in the ICU.

Rationale: The following factors existing before the onset of critical illness have been examined to determine if they affect sleep quality in ICU: female gender, older age, reported poor quality of sleep at home, regular use of sleep aid medication at home, and specific premorbid medical conditions (e.g., hypertension, diabetes, cancer, and thyroid disease) (Supplemental Table 43, Supplemental Digital Content 53, http://links.lww. $\mathrm{com} / \mathrm{CCM} / \mathrm{D} 811)$. Of these, only "reported poor quality sleep at home" $(459,482,483)$ and "regular use of a pharmacologic sleep aid at home" $(450,482)$ have been consistently reported in more than one study as being associated with perceived lower quality of sleep in the ICU.

\section{During ICU Admission.}

Question: Which ICU-acquired risk factors affect sleep quality in critically ill adults?

Ungraded Statement: Pain, environmental stimuli, healthcare-related interruptions, psychologic factors, respiratory factors, and medications each affect sleep quality in the ICU.

Rationale: Patient-perceived factors that contribute to patient-perceived poor sleep among critically ill adults have been reported according to either their severity (degree to which they disrupted sleep) or incidence (frequency with which they were reported) in 12 observational studies $(455,456,460$, 482, 484492) (Supplemental Table 44, Supplemental Digital Content 54, http://links.lww.com/CCM/D812). The factors most frequently cited by patients as disruptive to sleep were noise, pain and discomfort, immobility/restricted movement, nursing care interventions, and worry/anxiety/fear $(449,455,456,482,484-490$, $492,493)$. Four studies $(449,456,482,492)$ used the "Sleep in the Intensive Care Unit (ICU) Questionnaire" (455) to assess the severity of disruption caused by seven extrinsic (environmental) factors (ranked on a scale of 1-10 with 1 being no disruption and 10 significant disruption). The top three reported extrinsic factors disrupting sleep were noise, lighting, and nursing interventions (e.g., baths). All seven factors, including the top three, ranked 5 or less on the 10-point sleep disruptiveness scale (455, $482,484)$. When ICU patients were asked to rank 35 intrinsic and extrinsic factors on a $0-4$ scale (based on how disruptive each factor was to sleep), the top intrinsic factors were pain, inability to get comfortable, the bed, and procedures being performed on the patient $(456,485-490,492,493)$. A complete list of patient-identified factors is summarized in Table 2.

In addition to asking patients to identify factors they perceive as disruptive, other studies have measured sleep objectively using polysomnography or actigraphy and attempted to correlate risk factors with various measures of sleep. Factors that have been shown to correlate with sleep disruption in univariate analyses include illness severity (494), delirium $(442,443)$, hypoxemia and alkalosis (494), receiving a benzodiazepine (442) or propofol (464), patient-ventilator asynchrony (454), spontaneous (vs mechanically supported) breathing (452), and a spontaneous mode of ventilation (vs a controlled mode) $(472,495)$. Noise has been found to correlate temporally with arousals but appears to be responsible for only $10-17 \%$ of all arousals $(449,455,482$, 484). Only one study included multivariable analysis and found that presence of an endotracheal tube (i.e., receiving mechanical ventilation) seemed to confer improved sleep quality (460). The sleep of individual patients may be affected differently by various risk factors (e.g., some patients may be more bothered by noise than other patients) and the meaning or relevance to patients (e.g., some patients are comforted by hearing the nurse nearby, whereas others are bothered by it), and the patient's intrinsic disposition (e.g., susceptibility to feel worried, afraid, or uncomfortable under similar circumstances). 


\section{TABLE 2. List of Factors That Patients Report as Disruptive to Sleep}

Environmental

Noise (447, 453, 454, 480, 483-488, 490, 491)

Light (241, 453, 454, 480, 482-484, 486-488)

Comfort of bed $(483,486-488)$

Activities at other bedsides $(483,486,487)$

Visitors (clinician or family) (483)

Room ventilation system (483)

Hand washing by clinicians (483)

Bad odor $(486,488)$

Care Related

Nursing care (447, 453, 480, 482-484, 486, 488, 491)

Patient procedures $(447,453,480,482,483,487,488)$

Vital sign measurement $(442,448,475,477,481,483)$

Diagnostic tests $(447,453,480,483)$

Medication administration (447, 453, 480, 482)

Restricted mobility from lines/catheters $(454,486,488)$

Monitoring equipment $(454,486,488)$

Oxygen mask $(486,488)$

Endotracheal tube (491)

Urinary catheters (486)
Physiologic and Pathophysiologic

Pain (454, 483-486, 488, 490, 491)

Discomfort (454, 483, 486, 488, 490)

Feeling too hot or too cold $(484,486,488)$

Breathing difficulty $(484,491)$

Coughing $(484,491)$

Thirst $(484,486)$ and hunger $(486,488)$

Nausea $(484,488)$

Needing to use bedpan/urinal $(486,488)$

Psychologic

Anxiety/worry/stress (483, 484, 486, 489-491)

Fear $(485,486,489)$

Unfamiliar environment $(485,488,491)$

Disorientation to time $(454,486)$

Loneliness $(488,491)$

Lack of privacy $(485,488)$

Hospital attire $(486,488)$

Missing bedtime routine (483)

Not knowing nurses' names (486)

Not understanding medical terms (486)
Evidence Gaps: Studies using questionnaires and interviews, while patient-centered, are subject to recall bias and exclude patients who are not able to self-report due to sedation, delirium, dementia, or acute brain injury. Furthermore, patients may have sleep that is severely fragmented with microarousals, but the patients may not be able to identify the disruptive factors because they were not fully awakened from sleep. Studies using polysomnography are limited to those that can be analyzed by standard criteria and exclude highly abnormal electroencephalograms or those with poor-quality electroencephalogram signals. Studies correlating various factors with impaired sleep on polysomnography do not prove causation, only association, and were largely weak associations in univariate analysis.

\section{Outcomes}

Question: Do sleep and circadian rhythm alterations "during" an ICU admission affect outcomes during and/or after the ICU stay in critically ill adults?

Ungraded Statements: Although an association between sleep quality and delirium occurrence exists in critically ill adults, a cause-effect relationship has not been established.

An association between sleep quality and duration of mechanical ventilation, length of ICU stay, and ICU mortality in critically ill adults remains unclear.
The effects of sleep quality and circadian rhythm alterations on outcomes in critically ill patients after ICU discharge are unknown.

Rationale: A handful of studies help answer these questions (Supplemental Table 45, Supplemental Digital Content 55, http://links.lww.com/CCM/D813). Poor sleep quality is often assumed to be a potentially modifiable risk factor for ICU delirium; several studies have evaluated this relationship. Critically ill adults who are severely sleep deprived are 30\% more likely to have mental status changes (441). Subsequent polysomnography studies further supported this association (442, 451). Critically ill adults with severe REM deprivation (442, 451) and circadian sleep-cycle disruption (as evidenced by a greater proportion of daytime sleep) are more likely to experience delirium (451). Poor sleep quality has also been found to be an independent risk factor (496) for postcardiac surgery ICU delirium. Additionally, before-and-after observational studies of multidisciplinary bundles that include sleep enhancement protocols have been shown to decrease delirium prevalence $(312,454)$, although in only one study did sleep efficiency improve with the intervention (312). Although an association between sleep quality and delirium occurrence exists, it remains unknown if poor sleep is a cause for delirium.

Use of a multicomponent delirium prevention protocol that incorporated a nonpharmacologic sleep enhancement protocol 
was associated with shorter delirium duration and greater ventilator-free days (497). The relationship of sleep to these outcomes remains unclear as sleep was not measured in the study. Patients with abnormal sleep (increased daytime sleep; reduced REM) were more likely to fail NIV and require intubation and mechanical ventilation (443). In a small study of patients with moderate/ severe TBI, better or improving rest-activity cycle consolidation ( $\geq 80 \%$ daytime activity) was associated with shorter ICU and hospital stays (498). Among patients where abnormal sleep was felt to be a cause of late NIV failure, ICU stays were longer and both ICU and hospital mortality rates were greater (443). One quality improvement study showed no difference in mortality with its use despite delirium's being reduced (459). The presence of organized sleep patterns in patients with a recent TBI is predictive of improved survival (479). Although a number of studies have found that sleep remains disturbed after ICU discharge, no studies were found in the literature evaluating the effect of sleep in the ICU on outcomes after ICU discharge.

Evidence Gaps: Available studies cannot fully elucidate the relationship between sleep alterations in the critically ill and important outcomes such as delirium occurrence, duration of mechanical ventilation, ICU LOS, and mortality are inadequate to confirm whether an association exists between the sleep alterations seen in the critically ill adults and important outcomes such as delirium occurrence, duration of mechanical ventilation, ICU LOS, and mortality. Poor sleep may adversely affect the immune system, glycemic control, and the psychologic well-being of otherwise healthy individuals, so understanding if there are clinical effects on these and other outcomes in critically ill adults is of great importance. Studies that pair these outcomes with reliable measurement of sleep at the ICU bedside, while controlling for the multiple other factors that are associated with these outcomes, are needed. Additionally, studies are needed to determine the effects of ICU sleep quality on post-ICU outcomes.

\section{Monitoring}

Question: Should physiologic monitoring be routinely used clinically to evaluate sleep in critically ill adults?

Recommendation: We suggest not routinely using physiologic sleep monitoring clinically in critically ill adults (conditional recommendation, very low quality of evidence).

Remarks: Physiologic monitoring refers to the use of actigraphy, bispectral analysis (BIS), electroencephalography, and polysomnography to determine if a patient is asleep or awake. It specifically does "not" include monitoring of patients' perceived sleep by either validated assessment (e.g., the Richards Campbell Sleep Questionnaire) or informal subjective bedside assessment.

Rationale: None of the five critical outcomes chosen for this question (i.e., delirium occurrence, duration of mechanical ventilation, ICU LOS, ICU mortality, and patient satisfaction) have been studied. Observational studies have evaluated the role of physiologic sleep monitoring on other outcomes (Supplemental Table 46, Supplemental Digital Content 56, http://links.lww.com/CCM/D814). Physiologic monitoring identified sleep-disordered breathing in patients with acute coronary syndromes $(499,500)$, but the impact of this evaluation was not determined. When motor activity (as measured by actigraphy) was compared with nurses' sleep and sedation assessments in a small series of mechanically ventilated adults (501), limb movements were found to correlate with the measured neurologic indices. The use of polysomnography-derived electroencephalogram recordings of patients with nontraumatic (479) and traumatic (480) encephalopathy concluded that the presence of recognized elements of sleep was associated with a favorable prognosis. Finally, three small studies found that polysomnography can be used to optimize the method of mechanical ventilatory support in ICU patients in acute respiratory failure $(443,502,503)$. Despite these potential roles for polysomnography, its routine use in the ICU is not feasible.

The panel arrived at this recommendation based on the lack of high-quality evidence combined with the high cost of the resources necessary to implement most of the relevant technologies. Physiologic monitoring and interpretation have significant limitations as described above. Further, no studies investigated sleep monitoring in an unselected ICU population, thus calling into question the generalizability of the available data.

Although routine physiologic sleep monitoring is not recommended, we emphasize that clinicians "should" routinely inquire about patients' sleep or try to monitor it either by using one of the validated assessment tools such as the Richards Campbell Sleep Questionnaire or by informal bedside assessment. The Richard-Campbell Sleep Questionnaire has been shown to be a valid and reliable tool in critically ill adults to evaluate a patient's perception of their own sleep if they are both alert and oriented (504).

Poor sleep is considered to be one of the most common stresses experienced by critically ill patients $(435,437)$. Asking about patients' sleep may serve to validate patients' and their families' concerns and is a necessary first step to approaching an intervention. Nurse-observed sleep (439, 448, 505, 506) overestimated TST when compared with polysomnography evaluation. When nurse and patient perceptions of sleep are compared, the nurse may sometimes overestimate patients' perception of sleep quality $(485,492,505,507)$.

Evidence Gaps: How best to measure sleep in critically ill patients continues to be debated $(476,508)$. Routine monitoring of any brain activity in the ICU remains challenging. The problem of monitoring sleep is further complicated by the fact that the electrical activity of the brain alone (i.e., electroencephalogram) is insufficient to determine sleep stages, circadian activity, and sleep-disordered breathing. A simplified, generalizable system for monitoring sleep in the ICU that is resistant to the changing physiology of the critically ill patient and will stand up to regular use in the ICU setting would enhance our understanding of the relationship between sleep and ICU outcomes. In contrast to healthy individuals, critically ill patients have variation in vigilance states and electroencephalogram patterns not only due to natural sleep/wake states but also from sedating medications and delirium. Whether sedative-induced sleep provides the same restorative benefits as 
natural sleep is unknown. Large studies are needed to determine the best method of sleep measurement and classification and to measure how the individual factors (sleep, sedation, illness-induced encephalopathy) or a combination thereof affect patient outcomes, including patient satisfaction. The expense and time-consuming nature of polysomnography have made research of sleep measurement and sleep outcomes difficult. Other measurement techniques such as limited electroencephalogram and processed electroencephalogram devices may provide valuable data, but studies comparing them to polysomnography are needed to validate these methods.

\section{Nonpharmacologic Interventions to Improve Sleep}

A description of the ventilation modes evaluated in this section question and the methods used to identify studies and summarize data can be found in Supplemental Table 47 (Supplemental Digital Content 57, http://links.lww.com/CCM/D815).

\section{Ventilator Mode.}

Question: Should assist-control ventilation be used at night (vs pressure support ventilation) to improve sleep in critically ill adults?

Recommendation: We suggest using assist-control ventilation at night (vs pressure support ventilation) for improving sleep in critically ill adults (conditional recommendation, low quality of evidence).

Rationale: Many of the outcomes deemed critical or important by the panel for this question were not evaluated or reported. Pooled estimates of three studies $(n=61)(469,472$, 473 ) found that assist-control ventilation (vs pressure support ventilation) was associated with an increase in sleep efficiency (MD, 18.33\%; 95\% CI, 7.89-28.76; moderate quality). Although pooled estimates of two studies $(472,473)(n=41)$ found that assist-control ventilation (vs pressure support ventilation) was not associated with a difference in the percentage of TST spent in stage 1 (MD, $0.31 \%$; $95 \% \mathrm{CI},-5.17$ to 5.79 ; low quality) or stage $2(\mathrm{MD}, 5.29 \%$; $95 \% \mathrm{CI},-4.38$ to 14.97 ; very low quality) sleep, it was associated with more time spent in REM sleep (MD, 2.79\%; 95\% CI, 0.53-5.05; low quality). Although the quality was deemed to be low, given the potential benefits of this intervention, its low risk and the fact that all ventilators have assist-control mode capability, a conditional recommendation for using assist-control mode ventilation at night to improve sleep was made. For those patients who remain dyssynchronous despite all efforts to optimize ventilator settings on assist-control mode, however, clinicians will have to make a case-by-case decision whether to return the patient to pressure support ventilation or consider sedation, considering the deleterious effects of propofol and benzodiazepines on sleep quality and synchrony.

Question: Should an adaptive mode of ventilation be used at night (vs pressure support ventilation) to improve sleep in critically ill adults?

Recommendation: We make no recommendation regarding the use of an adaptive mode of ventilation at night (vs pressure support ventilation) for improving sleep in critically ill adults (no recommendation, very low quality of evidence).
Rationale: Five small randomized controlled crossover trials compared an adaptive mode of ventilation versus a pressure support mode in primarily medical critically ill adults, evaluating outcomes the panel deemed important but none were deemed critical. The adaptive modes studied were as follows: automatically adjusted pressure support (473), proportional assist ventilation (462), proportional assist ventilation with load-adjustable gain factors $(468,471)$, and neutrally adjusted ventilator assist (465). Feasibility may also be a concern because some ICUs might not have ventilators or staff trained to deliver an adaptive ventilation mode. Based on these issues, and the reluctance to issue a recommendation based on this small, single-center study due to feasibility/availability concerns in other centers, we were not able to make a recommendation regarding the use of adaptive ventilation at night.

\section{NIV-Dedicated Ventilator.}

Question: Among critically ill adults requiring NIV, should an NIV-dedicated ventilator (vs a standard ICU ventilator with NIV capacity) be used to improve sleep?

Recommendation: We suggest using either an NIV-dedicated ventilator or a standard ICU ventilator for critically ill adults requiring NIV to improve sleep (conditional recommendation, very low quality of evidence).

Rationale: Only one small randomized trial was available to answer this question, and it did not evaluate most of the defined outcomes (454). No significant differences appeared between use of an NIV-dedicated ventilator and a standard ICU ventilator with respect to sleep efficiency; percent of time spent in stage 1 , stage 2 , stage $3 / 4$, or REM sleep; or sleep fragmentation index. Compared with periods off NIV, sleep during NIV resulted in increased REM and stage 3/4 sleep with a reduction in sleep fragmentation index. Based on the above, patients with acute hypercapnic respiratory failure have improved sleep quality during NIV compared with without NIV, but we recommend that either type of ventilator, dependent on feasibility and convenience, is acceptable to use for ICU patients requiring NIV.

Evidence Gaps: Studies comparing sleep between assisted breathing and controlled breathing in the assist-control and adaptive modes and the adaptive mode and assist-control ventilation modes have not been published. Studies used polysomnography to measure sleep on various modes, but none of these studies evaluated patients' perception of their sleep.

\section{Aromatherapy/Acupressure/Music}

Question: Should aromatherapy, acupressure, or music be used at night (vs not using it) to improve sleep in critically ill adults?

Recommendation: We suggest not using aromatherapy, acupressure, or music at night to improve sleep in critically ill adults (conditional recommendation, low quality of evidence [aromatherapy and acupressure]; very low quality of evidence [music]).

Rationale: Two small, unblinded RCTs $(509,510)$ evaluated the use of aromatherapy for improving sleep in conscious and communicative ICU patients. No adverse effects were reported, but a pooled analysis demonstrated no effect with its use (vs no 
use) in patient-reported sleep quality (MD, 0.02 points; $95 \%$ CI, -0.36 to 0.41 ; low quality) and the overall quality of evidence was low. Although a low-cost intervention that is generally considered safe, the lack of proven benefit for sleep in addition to some concern about using potential respiratory irritants in an ICU population led the panel to make a conditional recommendation against aromatherapy in the ICU.

One small RCT $(n=85)$ (511) evaluated the use of acupressure in ICU patients having a low severity of illness. Researchers who attended an acupressure training course applied pressure for 3 minutes to each of six acupoints between 7:00 PM and 10:00 PM and found that acupressure (vs no use of acupressure) was associated with an increased duration of sleep when evaluated by actigraphy (MD, $0.5 \mathrm{hr} ; 95 \% \mathrm{CI}, 0.09-0.91$; low quality) or the nurse (MD, $1.1 \mathrm{hr}$; 95\% CI, 0.39-1.81; low quality) and less daytime sleepiness on the Stanford Sleepiness Scale (MD, 0.4 points; $95 \%$ CI, $0.66-0.14$; low quality). Given the high risk of bias for the single included study, the small number of patients enrolled, the cost of having a trained clinician provide acupressure, and the lack of availability of this modality at many centers, we decided to suggest against the use of acupressure to improve sleep in critically ill adults. For those institutions with trained personnel and expertise, however, it may be a reasonable intervention, especially if requested by patients.

One small RCT $(n=28)$ (512) evaluated the effect of playing music on the piano (four sedating pieces lasting $45 \mathrm{~min}$ ) on sleep outcomes (during first $2 \mathrm{hr}$ of night) in critically ill adults. The music had a small effect on improving sleep quality (as evaluated by the Verran and Snyder-Halpern Sleep Scale) (MD, 48 points; $95 \%$ CI, 34.5-130.5; very low quality) and sleep efficiency (as evaluated by polysomnography) (MD, 2.3\%; 95\% CI, 27.3-32.0; very low quality). Given the low quality of evidence (no blinding, ambient noise not controlled) and the resources needed to institute this intervention, the panel made a conditional recommendation against the use of music to improve sleep in critically ill adults. Music may play a role in reducing pain (see pain section) and anxiety in the ICU (133). If patients (or their families) request it, it should be considered.

\section{Noise and Light Reduction}

Question: Should noise and light reduction strategies (vs not using these strategies) be used at night to improve sleep in critically ill adults?

Recommendation: We suggest using noise and light reduction strategies to improve sleep in critically ill adults (conditional recommendation, low quality of evidence).

Rationale: Two RCTs $(458,513)$ and two observational studies $(514,515)$ evaluated strategies to reduce ICU noise and light at night through the use of earplugs with or without the use of eyeshades. Use of earplugs and eyeshades (vs control) on the first postoperative ICU night after cardiac surgery maintained sleep quality at the preoperative level (513). Application of earplugs (vs no earplugs) to nonsedated, critically ill adults improved patient-reported sleep quality and reduced delirium (458). Pooled analysis from the two ICU observational studies $(n=164)$ found that application of ear plugs (vs no ear plugs) was associated with a greater proportion of achieving greater than 4 hours of sleep (RR, 1.2; 95\% CI, 0.64-2.24; low quality) $(513,515)$. The overall quality of evidence was low due to a lack of blinding, a population of patients not severely ill, and the refusal of some patients to keep the earplugs inserted. Earplugs, with or without eyeshades, represent a low-cost intervention that can be applied in all ICUs to improve sleep quality and reduce delirium. In general patients, particularly those who cannot initiate sleep, should be asked if they want this intervention and earplugs should always be removed in the morning.

Evidence Gaps: Nonpharmacologic strategies focused on improving sleep in the ICU need to be evaluated in large randomized trials, include ICU patients with higher severity of illness, and rigorously evaluate the effect of these interventions on sleep quality. The group of patients in the ICU who may gain the most benefit from these interventions needs to be elucidated.

\section{Pharmacologic Interventions to Improve Sleep}

Given the challenges of promoting naturally occurring sleep in the ICU, patients and their family members may ask for sleepenhancing medication. Although their request should always be considered, this pressure and our efforts to provide compassionate care sometimes lead to the administration of medications that are poorly tested for safety and efficacy in ICU patients and that may increase the risk for polypharmacy and delirium rather than actually promote sleep. Pharmacologic interventions were considered by drug type/class and were reviewed by the panel solely for their effect on sleep promotion.

Question: Should a sleep-promoting medication (i.e., melatonin, dexmedetomidine, or propofol) (vs no use of a medication) be used to improve sleep in critically ill adults?

\section{Melatonin.}

Recommendation: We make no recommendation regarding the use of melatonin to improve sleep in critically ill adults (no recommendation, very low quality of evidence).

Rationale: Three small, placebo-controlled, randomized trials $(n=60)$ evaluating the night-time administration of melatonin were reviewed. The first found that the administration of $10 \mathrm{mg}$ of melatonin at night (vs placebo) to 12 patients in the ICU having chronic respiratory failure was associated with nonsignificant improvements in both sleep quality and quantity (as evaluated by BIS) (516). A second RCT that evaluated night-time melatonin $3 \mathrm{mg}$ (or placebo) in 16 patients in a similar population and evaluated sleep using actigraphy arrived at a similar conclusion (517). A third RCT that compared melatonin $3 \mathrm{mg}$ (or placebo) to 32 patients who also were admitted to the ICU with chronic respiratory failure as the first two studies found no discernible difference in the duration of "observed nocturnal sleep" by bedside nurse assessment (518). The limitations of evaluating sleep in the ICU using BIS, actigraphy, or subjective nursing scales rather than polysomnography are highlighted previously in the guidelines. 
The manufacture of melatonin in the United States is not Food and Drug Administration regulated; concerns as to the quality and consistency of the product (519) have prevented many hospitals from adding it to their formulary. Melatonin is, however, associated with relatively few adverse effects (e.g., mild sedation and headache) and inexpensive. The panel decided on no recommendation due to the perceived balance between desirable and undesirable outcomes and the lack of high-quality evidence.

Ramelteon, an FDA-approved melatonin receptor agonist, was evaluated in a single study (not included in this analysis) to prevent delirium in the elderly (520). A small number of patients in that study were critically ill; however, there was no demonstrable improvement in subjective sleep quality. Similar to melatonin, few adverse events are reported with the medication, but sleep promotion was not proven and the cost is higher than that of melatonin. One recent single-center, double-blind, placebo-controlled RCT, also not included in this analysis, found that the administration of $8 \mathrm{mg}$ of ramelteon at 20:00 hours each day to critically ill adults without delirium was associated with significant reduction in delirium occurrence (521).

\section{Dexmedetomidine.}

Recommendation: We make no recommendation regarding the use of dexmedetomidine at night to improve sleep (no recommendation, low quality of evidence).

Rationale: Two randomized trials $(n=74)$ evaluated the effects of dexmedetomidine in critically ill, mechanically ventilated adults requiring sedation (470) and in critically ill, nonmechanically ventilated patients not requiring a continuous infusion of a sedative medication (521). Both studies demonstrated that dexmedetomidine increased stage 2 sleep (MD, $47.85 \% \mathrm{~min}$; $95 \% \mathrm{CI}, 24.05-71.64$; moderate quality) and decreased in stage 1 sleep (MD, $-30.37 \%$; $95 \% \mathrm{CI},-50.01$ to -10.73 ; moderate quality), each of which the panel considered favorable outcomes $(470,521)$. Neither study, however, demonstrated a decrease in sleep fragmentation or an increase in deep sleep or REM sleep that are thought to be the most restorative sleep stages and thus potentially most important to recovery. A third, observational trial, not included in this analysis, corroborated these findings with regard to sleep architecture and noted preserved day-night cycling when dexmedetomidine was administered overnight in mechanically ventilated ICU patients (522). One recently published double-blind, placebocontrolled RCT of 100 delirium-free critically ill adults receiving sedatives, and not included in the evidence profile, found that the administration of low-dose dexmedetomidine did not change Leeds Sleep Evaluation Questionnaire scores between the dexmedetomidine and placebo groups (370).

Consideration was given to a conditional recommendation in favor of using dexmedetomidine at night for the sole purpose of sleep promotion; however, clinical concerns include its high cost, hemodynamic side effects, and generalizability of the existing studies. If a sedative infusion is indicated for a hemodynamically stable, critically ill adult overnight, dexmedetomidine may be a reasonable option because of its potential to improve sleep architecture (523). See the sedation section for a more indepth evaluation of sedative choice in critically ill adults.

\section{Propofol.}

Recommendation: We suggest not using propofol to improve sleep in critically ill adults (conditional recommendation, low quality of evidence).

Rationale: Two RCTs compared propofol with benzodiazepines $(454,524)$, and one compared propofol with placebo (525). No demonstrable improvement in sleep occurred with propofol compared with placebo. Further, propofol was associated with REM suppression, hemodynamic side effects, and respiratory depression sometimes necessitating mechanical ventilation. Although we recommend against using propofol for the sole purpose of improving sleep in the critically ill, this recommendation does not intend to address its use in patients requiring procedural or continuous sedation.

Other medications administered with the intent to improve sleep in the critically ill include tricyclic antidepressants, atypical antipsychotics, and hypnotics such as benzodiazepines and benzodiazepine-receptor agonists. Currently, there is insufficient information to consider a recommendation for any of medications to help promote sleep in the critically ill. Although their adverse effects are well described, their benefits in terms of sleep promotion are unknown.

Evidence Gaps: Large, well-controlled trials of medications administered at night for the sole purpose of sleep promotion in critically ill patients are lacking. This is especially true for medications such as tricyclic antidepressants and atypical antipsychotics that are frequently used for this purpose because they are less likely to precipitate an episode of delirium, have fewer hemodynamic and respiratory depressant effects, and because their sedating side effects suggest the possibility of sleep promotion. These medications, however, should be rigorously studied to assess their efficacy in this population to determine if the benefits justify their potential harms.

\section{Sleep-Promoting Protocol}

Question: Should a sleep-promoting protocol be used to improve sleep in critically ill adults?

Recommendation: We suggest using a sleep-promoting, multicomponent protocol in critically ill adults (conditional recommendation, very low quality of evidence.)

Rationale: Protocols are a common way to incorporate multiple interventions at once into a clinical practice guideline (526), including those described below for sleep quality improvement in critically ill patients. The sleep-promoting protocols eligible for inclusion varied in their components, as described below $(459,527-529)$. All included offering earplugs and eyeshades to patients who could choose to use them or to discontinue their use if they wished and two also included use of relaxing music $(459,526)$. Among the two composed of a more complex combination of interventions, one specified a pharmacologic guideline that discouraged the use of sedating medications known to alter sleep and/or precipitate delirium 
and introduced interventions in stages over a 5-month period (459). In all studies, protocols were applied to all ICU patients and did not target a subset of patients known to have poor sleep quality.

The critical outcomes examined were sleep stages, sleep duration, sleep fragmentation, circadian rhythm, delirium, duration of mechanical ventilation, mortality, LOS (ICU and hospital), and patient experience. Current published data contain four studies reporting outcomes relevant to this question, one RCT (527), and three observational studies $(459,528,529)$ (Supplemental Table 48 Supplemental Digital Content 58, http://links.lww.com/CCM/D816). One small RCT in open-heart surgery patients demonstrated that earplugs, eyeshades, and relaxing music improved selfreported sleep quality (528). Among the three observational before-and-after studies, one found an improvement in sleep in a mixed ICU population (529), whereas the other two did not $(459,528)$. Pooled analysis of the three studies demonstrated an overall reduction in the prevalence of delirium with a sleep-promoting protocol (RR, 0.62 ; 95\% CI, $0.42-$ 0.91 ; very low quality). One of the observational studies used a similar intervention to $\mathrm{Hu}$ et al (527), earplugs, eye shades, and music, whereas the other two tested more complex interventions including these interventions plus environmental changes, namely clustering of care to minimize interruptions overnight and early mobilization $(459,529)$. One study also specifically included pharmacologic guidelines, administering zolpidem to patients without delirium and haloperidol or an atypical antipsychotic for patients with delirium (459). In an effort to minimize the influence of medications on outcomes, Patel et al (529) excluded patients who had received sedatives in the 24 hours before enrollment. Which of the interventions, or which combinations of the interventions, are effective in improving sleep and reducing delirium cannot be discerned from the above studies. Overall evidence was low or very low quality due to risk of confounding, imprecision, and the potential for risk of bias in the included studies. The panel made a conditional recommendation based on the potential for benefit (e.g., delirium reduction) and minimal anticipated harm. The panel recognized, however, that implementing and sustaining multifaceted clinical practice protocols can be resource intensive (530).

Evidence Gaps: Future research should investigate which of the interventions, or which combinations of the interventions, are effective in improving sleep and reducing delirium. The effect on reduction in delirium in the reviewed studies but less demonstrable on sleep quality is notable, reinforcing that more work on the assessment of sleep in critically ill adults is needed, as recommended above. Although many thousands of publications on the science of implementing evidenced-based clinical practice guidelines exist, relatively few address improving sleep in critically ill adult patients; this specific topic would benefit from further investigation. Mortality, ICU LOS, and duration of mechanical ventilation were reported in the reviewed studies, but numbers were too small to draw any conclusions.
These, as well as patient experience and patient-centered mid- to long-term outcomes such as sleep quality, psychologic health, and quality of life determinants such as autonomous living remain unexplored.

Concluding Comments on Sleep: Studies to date are consistent in demonstrating that critically ill patients sleep poorly as a result of both patient and ICU factors. The importance of improving sleep in this population may be unproven by RCT but is intuitive and, at least, could be considered an important comfort measure that would improve patients' ICU quality of life if not other outcomes. Although only a select few intervention studies have been published, available data suggest that a multicomponent protocolized approach to improving sleep that favors nonpharmacologic measures may offer our patients their best chance for a better night's sleep. Future research needs to focus on improved methods for measuring sleep and on implementing interventions targeting patient-centered outcomes. Sleep habits are highly variable among healthy individuals; therefore, a more individualized approach should be considered.

\section{SUMMARY}

Thousands of hours were invested by these guidelines' authors, who were in turn supported by formal and informal collaborators, over the 3.5 years it took to produce this effort. As experts mandated by the Society of Critical Care Medicine, we aimed to provide the recent information clinicians need to better care for critically ill adults $(531,532)$ using the most rigorous and transparent processes at our disposition. Because such process does not necessarily ensure acceptability among knowledge providers and users (533), we established ways in which to address relevant and patient-centered pain, sedation, delirium, immobility, and sleep practice-related questions. The diversity of our experts (534), representing many professions on three continents, generated vigorous discussions as to clinical approaches and care aspects that differed by geographical availability (of medication interventions, for instance) and by institutional culture. Because we did not limit our reviews to English language publications, the evidence gathered to support our recommendations represents literature from around the world.

The recommendation rationales, fueled by debate and discussion, circled back to the bedside experience-and the perspective of what was best for patients-held by all panelists and methodology experts. In sections added to these guidelines since their last 2013 version (1) (rehabilitation/mobility and sleep), we sought to clarify conceptual definitions within these relatively new critical care research domains. We wanted to make them accessible to facilitate incorporating them into the complex patient management reasoning any critical care clinician might consider. We challenged common practices such as administering antipsychotics to delirious patients. We invited clinicians to expand the proposed interventions in comparison to the 2013 guidelines (1); one example is the consideration of multiple pharmacologic and nonpharmacologic coanalgesic approaches to the ICU patient. When the published evidence 
was insufficient, limited to a narrow population or specific intervention (e.g., for procedural analgesia), or outright absent to answer the questions we posed, we structured evidence gap descriptors to inform clinicians where the uncertainty lay, and intended to provide sufficient information to apprise and invite researchers to address these gaps.

We are mindful of the limitations inherent to our work. "Good evidence" requirements for randomized trials involving many patients have its caveats; practice misalignment (535) and diagnostic confounders (312) were, to the extent it was possible, considered, but "unknown" factors with the potential to influence evidence likely exist. One example is the recent introduction of stratification by frailty (536) in trials involving the critically ill, which could not be considered because this comorbidity had not been taken into account in much of the literature justifying our recommendations. Another is the fact that although all patients were admitted to an ICU, both the reasons leading to their ICU admission and severity of illness varied considerably, warranting individual tailoring of our recommendations to individual patient considerations. A degree of uncertainty is as inherent to clinical practice as it is to the research process and its resulting conclusions (537). The quest to make our decision making and iterative innovations transparent and accessible motivated the methods article that was prepared separately from this guideline initiative (13).

Finally, the development of guidelines like these does not ensure their use (538). Some educational programs and the provision of feedback in relationship to attaining analgesia and sedation-targeted performance goals have been disappointingly ineffective when studied prospectively $(3,4)$. We consider the effectiveness and limitations of different dissemination methods and approaches germane to this guideline's topics in a separate publication as a tool to inform educational programming and quality improvement initiatives that will evolve from this guideline (2). In addition to bridging the gap between the knowledge we gathered and its application, we believe that this will provide tangible support to clinicians, stakeholders, and decision makers in implementing quality in pain, agitation, delirium, early mobility, and sleep and further foster the application of what we understand to be useful in the provision and delivery of excellent care.

\section{ACKNOWLEDGMENTS}

We acknowledge the many direct and indirect contributors to this effort: Margaret McIvor, an ICU survivor whose contribution was limited by subsequent illness; students, trainees, and colleagues (Julie C. Reid, PT, MSc; Anastasia Newman, PT, MSc; David J. Gagnon, PharmD; Lauren E. Payne, PharmD; Nicole Kovacic, PharmD; Kimia Honarmand, MD, MSc; Jamie Le, MD; Sindu Mohan, MD; Peter J. Hurh, MD; Justin D. Dumont, DO, MS; M. Farhan Nasser, MD; Venkat R. Venna, MD; Aparna Nallagangula, MBBS; Kimberly J. Terry, PharmD; and Jeremy R. DeGrado, PharmD) helped with abstract and full-text screening, supervised by several of the authors; Grading of Recommendations Assessment, Development and
Evaluation group members (Fayez Alshamsi, MD) who provided help with data analyses; Charlie Kishman, MSL, who initiated the literature searches as a continuation of his contribution to the 2013 PAD guidelines; Matt Duprey, PharmD, for his valuable support at the 2017 Hawaii meeting; Lori Harmon and Sylvia Quintanilla who provided direction and organizational infrastructure; and Deb McBride copywrote and edited the final article. The panel coauthors' effort would not have been possible without the explicit and implicit support of colleagues, families, and friends. The time committed to the Pain, Agitation/sedation, Delirium, Immobility (rehabilitation/ mobilization), and Sleep (disruption) initiative had to be weighed against availability to attend personal and professional challenges. We wish to acknowledge all those who shouldered other responsibilities, indirectly facilitating the creation and writing of these guidelines. Finally, we wish to thank the patients, teachers, and colleagues who inspired this effort and who challenged us to honor, and rise to the challenge of, this academic effort.

\section{REFERENCES}

1. Barr J, Fraser GL, Puntillo K, et al; American College of Critical Care Medicine: Clinical practice guidelines for the management of pain, agitation, and delirium in adult patients in the intensive care unit. Crit Care Med 2013; 41:263-306

2. Balas MC, Weinhouse GL, Denehy L, et al: Interpreting and implementing the 2018 Pain, Agitation/Sedation, Delirium, Immobility, and Sleep Disruption Clinical Practice Guideline. Crit Care Med 2018; 46:1464-1470

3. Walsh TS, Kydonaki K, Antonelli J, et al; Development and Evaluation of Strategies to Improve Sedation Practice in Intensive Care (DESIST) Study Investigators: Staff education, regular sedation and analgesia quality feedback, and a sedation monitoring technology for improving sedation and analgesia quality for critically ill, mechanically ventilated patients: A cluster randomised trial. Lancet Respir Med 2016; 4:807-817

4. Khan BA, Fadel WF, Tricker JL, et al: Effectiveness of implementing a wake up and breathe program on sedation and delirium in the ICU. Crit Care Med 2014; 42:e791-e795

5. Skrobik $\mathrm{Y}$, Ahern S, Leblanc M, et al: Protocolized intensive care unit management of analgesia, sedation, and delirium improves analgesia and subsyndromal delirium rates. Anesth Analg 2010; 111:451-463

6. Kamdar BB, Yang J, King LM, et al: Developing, implementing, and evaluating a multifaceted quality improvement intervention to promote sleep in an ICU. Am J Med Qual 2014; 29:546-554

7. Balas MC, Burke WJ, Gannon D, et al: Implementing the awakening and breathing coordination, delirium monitoring/management, and early exercise/mobility bundle into everyday care: Opportunities, challenges, and lessons learned for implementing the ICU Pain, Agitation, and Delirium Guidelines. Crit Care Med 2013; 41:S116-S127

8. Hoyer EH, Friedman M, Lavezza A, et al: Promoting mobility and reducing length of stay in hospitalized general medicine patients: $A$ quality-improvement project. J Hosp Med 2016; 11:341-347

9. Barnes-Daly MA, Phillips G, Ely EW: Improving hospital survival and reducing brain dysfunction at seven California community hospitals: Implementing PAD guidelines via the ABCDEF bundle in 6,064 patients. Crit Care Med 2017; 45:171-178 
10. Selva A, Sanabria AJ, Pequeño S, et al: Incorporating patients' views in guideline development: A systematic review of guidance documents. J Clin Epidemio/ 2017; 88:102-112

11. Guyatt $G H$, Oxman AD, Vist GE, et al; GRADE Working Group: GRADE: An emerging consensus on rating quality of evidence and strength of recommendations. BMJ 2008; 336:924-926

12. Alonso-Coello P, Oxman AD, Moberg J, et al; GRADE Working Group: GRADE Evidence to Decision (EtD) frameworks: A systematic and transparent approach to making well informed healthcare choices. 2: Clinical practice guidelines. BMJ 2016; 353:i2089

13. Devlin JW. Skrobik Y, Rochwerg B, et al: Methodological Innovation in Creating Clinical Practice Guidelines: Insights From the 2018 Society of Critical Care Medicine Pain, Agitation/Sedation, Delirium, Immobility, and Sleep Disruption Guideline Effort. Crit Care Med 2018; 46:1457-1463

14. The Joint Commission: New and revised standards related to pain assessment and management. 2017. Available at: https://www. jointcommission.org/assets/1/18/Joint_Commission_Enhances_ Pain_Assessment_and_Management_Requirements_for_Accredited_Hospitals1.PDF. Assessed January 5, 2018

15. Chanques G, Sebbane M, Barbotte E, et al: A prospective study of pain at rest: Incidence and characteristics of an unrecognized symptom in surgical and trauma versus medical intensive care unit patients. Anesthesiology 2007; 107:858-860

16. Puntillo KA, Max A, Timsit JF, et al: Determinants of procedural pain intensity in the intensive care unit. The Europain ${ }^{\circledR}$ study. Am J Respir Crit Care Med 2014; 189:39-47

17. Loeser JD, Treede RD: The Kyoto protocol of IASP basic pain terminology. Pain 2008; 137:473-477

18. McCaffery M, Alexander Beebe A: Pain: Clinical Manual for Nursing Practice. St. Louis, Mosby, 1994

19. Merskey H, Bogduk N; Task Force on Taxonomy of the International Association for the Study of Pain: Classification of Chronic Pain: Descriptions of Chronic Pain Syndromes and Definitions of Pain Terms. Seattle, WA, IASP Press, 1994

20. Vervest AC, Schimmel GH: Taxonomy of pain of the IASP. Pain 1988; 34:318-321

21. de Jong $A$, Molinari $N$, de Lattre $S$, et al: Decreasing severe pain and serious adverse events while moving intensive care unit patients: $A$ prospective interventional study (the NURSE-DO project). Crit Care 2013; 17:R74

22. Georgiou E, Hadjibalassi M, Lambrinou E, et al: The impact of pain assessment on critically ill patients' outcomes: A systematic review. Biomed Res Int 2015; 2015:503830

23. Puntillo KA, Naidu R: Chronic pain disorders after critical illness and ICU-acquired opioid dependence: Two clinical conundra. Curr Opin Crit Care 2016; 22:506-512

24. Macintyre PE, Huxtable CA, Flint SL, et al: Costs and consequences: A review of discharge opioid prescribing for ongoing management of acute pain. Anaesth Intensive Care 2014; 42:558-574

25. Yi P, Pryzbylkowski P: Opioid induced hyperalgesia. Pain Med 2015; 16(Suppl 1):S32-S36

26. Carroll KC, Atkins PJ, Herold GR, et al: Pain assessment and management in critically ill postoperative and trauma patients: A multisite study. Am J Crit Care 1999; 8:105-117

27. Puntillo K, Weiss SJ: Pain: Its mediators and associated morbidity in critically ill cardiovascular surgical patients. Nurs Res 1994; 43:3136

28. Al Sutari MM, Abdalrahim MS, Hamdan-Mansour AM, et al: Pain among mechanically ventilated patients in critical care units. J Res Med Sci 2014; 19:726-732

29. Navarro-García MA, Marín-Fernández B, de Carlos-Alegre $V$, et al: Preoperative mood disorders in patients undergoing cardiac surgery: Risk factors and postoperative morbidity in the intensive care unit. Rev Esp Cardiol (English Edition) 2011; 64:1005-1010

30. Desbiens NA, Wu AW, Broste SK, et al: Pain and satisfaction with pain control in seriously ill hospitalized adults: Findings from the SUPPORT research investigations. For the SUPPORT investigators. Study to understand prognoses and preferences for outcomes and risks of treatment. Crit Care Med 1996; 24:1953-1961
31. Arbour $C$, Choinière $M$, Topolovec-Vranic J, et al: Detecting pain in traumatic brain injured patients exposed to common procedures in the ICU: Typical or atypical behaviors. Clin J Pain 2014; 30:960969

32. Puntillo KA, Morris AB, Thompson CL, et al: Pain behaviors observed during six common procedures: Results from Thunder Project II. Crit Care Med 2004; 32:421-427

33. Faigeles B, Howie-Esquivel J, Miaskowski C, et al: Predictors and use of nonpharmacologic interventions for procedural pain associated with turning among hospitalized adults. Pain Manag Nurs 2013; 14:85-93

34. Stotts NA, Puntillo K, Bonham Morris A, et al: Wound care pain in hospitalized adult patients. Heart Lung 2004; 33:321-332

35. Stotts NA, Puntillo K, Stanik-Hutt J, et al: Does age make a difference in procedural pain perceptions and responses in hospitalized adults? Acute Pain 2007; 9:125-134

36. Stanik-Hutt JA, Soeken KL, Belcher AE, et al: Pain experiences of traumatically injured patients in a critical care setting. Am J Crit Care $2001 ; 10: 252-259$

37. Arroyo-Novoa CM, Figueroa-Ramos MI, Puntillo KA, et al: Pain related to tracheal suctioning in awake acutely and critically ill adults: A descriptive study. Intensive Crit Care Nurs 2008; 24:20-27

38. Puntillo K, Gélinas C, Chanques G: Next steps in ICU pain research. Intensive Care Med 2017; 43:1386-1388

39. Chanques G, Viel E, Constantin JM, et al: The measurement of pain in intensive care unit: Comparison of 5 self-report intensity scales. Pain 2010; 151:711-721

40. Gélinas C: Management of pain in cardiac surgery ICU patients: Have we improved over time? Intensive Crit Care Nurs 2007; 23:298-303

41. Rahu MA, Grap MJ, Ferguson P, et al: Validity and sensitivity of 6 pain scales in critically ill, intubated adults. Am J Crit Care 2015; 24:514-523

42. Karahan A, Ersayın A, Yildırım F, et al: Comparison of three rating scales for assessing pain intensity in an intensive care unit. Turk $J$ Thorac Cardiovasc Surg 2012; 20:50-55

43. Gélinas C: The Faces Pain Thermometer: A new tool for critically ill adults. Perspect Infirm 2007; 4:12-20

44. Gélinas C, Klein K, Naidech AM, et al: Pain, sedation, and delirium management in the neurocritically ill: Lessons learned from recent research. Semin Respir Crit Care Med 2013; 34:236-243

45. Terai T, Yukioka $\mathrm{H}$, Asada A: Pain evaluation in the intensive care unit: Observer-reported faces scale compared with self-reported Visual Analog Scale. Reg Anesth Pain Med 1998; 23:147-151

46. Rahu MA, Grap MJ, Cohn JF, et al: Facial expression as an indicator of pain in critically ill intubated adults during endotracheal suctioning. Am J Crit Care 2013; 22:412-422

47. Paulson-Conger M, Leske J, Maidl C, et al: Comparison of two pain assessment tools in nonverbal critical care patients. Pain Manag Nurs $2011 ; 12: 218-224$

48. Gélinas C, Puntillo KA, Levin P, et al: The Behavior Pain Assessment Tool for critically ill adults: A validation study in 28 countries. Pain 2017; 158:811-821

49. Gélinas C, Arbour C, Michaud C, et al: Patients and ICU nurses' perspectives of non-pharmacological interventions for pain management. Nurs Crit Care 2013; 18:307-318

50. Dehghani H, Tavangar H, Ghandehari A: Validity and reliability of Behavioral Pain Scale in patients with low level of consciousness due to head trauma hospitalized in intensive care unit. Arch Trauma Res 2014; 3:e18608

51. Yu A, Teitelbaum J, Scott J, et al: Evaluating pain, sedation, and delirium in the neurologically critically ill-feasibility and reliability of standardized tools: A multi-institutional study. Crit Care Med 2013; 41:2002-2007

52. Echegaray-Benites C, Kapoustina O, Gélinas C: Validation of the use of the Critical-Care Pain Observation Tool (CPOT) with brain surgery patients in the neurosurgical intensive care unit. Intensive Crit Care Nurs 2014; 30:257-265 
53. Joffe AM, McNulty B, Boitor M, et al: Validation of the Critical-Care Pain Observation Tool in brain-injured critically ill adults. J Crit Care 2016; 36:76-80

54. Lee J, Jung J, Noh JS, et al: Perioperative psycho-educational intervention can reduce postoperative delirium in patients after cardiac surgery: A pilot study. Int J Psychiatry Med 2013; 45:143-158

55. Li Q, Wan X, Gu C, et al: Pain assessment using the Critical-Care Pain Observation Tool in Chinese critically ill ventilated adults. J Pain Symptom Manage 2014; 48:975-982

56. Kwak EM, Oh H: Validation of a Korean translated version of the Critical Care Pain Observation Tool (CPOT) for ICU patients. J Korean Acad Nurs 2012; 42:76-84

57. Vázquez M, Pardavila MI, Lucia M, et al: Pain assessment in turning procedures for patients with invasive mechanical ventilation. Nurs Crit Care $2011 ; 16: 178-185$

58. Nürnberg Damström D, Saboonchi F, Sackey PV, et al: A preliminary validation of the Swedish version of the Critical-Care Pain Observation Tool in adults. Acta Anaesthesiol Scand 2011; 55:379-386

59. Liu Y, Li L, Herr K: Evaluation of two observational pain assessment tools in Chinese critically ill patients. Pain Med 2015; 16:1622-1628

60. Puntillo KA, Neuhaus J, Arai S, et al: Challenge of assessing symptoms in seriously ill intensive care unit patients: Can proxy reporters help? Crit Care Med 2012; 40:2760-2767

61. Bae KH, Jeong IS: Pain perception of nurses and pain expression of patients in critical care units. J Korean Acad Nurs 2014; 44:437-445

62. Desbiens NA, Mueller-Rizner N: How well do surrogates assess the pain of seriously ill patients? Crit Care Med 2000; 28:1347-1352

63. Aïssaoui $Y$, Zeggwagh AA, Zekraoui A, et al: Validation of a Behavioral Pain Scale in critically ill, sedated, and mechanically ventilated patients. Anesth Analg 2005; 101:1470-1476

64. Arbour C, Gélinas C: Are vital signs valid indicators for the assessment of pain in postoperative cardiac surgery ICU adults? Intensive Crit Care Nurs 2010; 26:83-90

65. Boitor M, Martorella G, Arbour C, et al: Evaluation of the preliminary effectiveness of hand massage therapy on postoperative pain of adults in the intensive care unit after cardiac surgery: A pilot randomized controlled trial. Pain Manag Nurs 2015; 16:354-366

66. Chanques G, Payen JF, Mercier G, et al: Assessing pain in non-intubated critically ill patients unable to self report: An adaptation of the Behavioral Pain Scale. Intensive Care Med 2009; 35:2060-2067

67. Chen HJ, Chen YM: Pain assessment: Validation of the physiologic indicators in the ventilated adult patient. Pain Manag Nurs 2015; 16:105-111

68. Gélinas $C$, Arbour C: Behavioral and physiologic indicators during a nociceptive procedure in conscious and unconscious mechanically ventilated adults: Similar or different? J Crit Care 2009; 24:628.e7-628.e17

69. Gélinas C, Johnston C: Pain assessment in the critically ill ventilated adult: Validation of the Critical-Care Pain Observation Tool and physiologic indicators. Clin J Pain 2007; 23:497-505

70. Kapoustina O, Echegaray-Benites C, Gélinas C: Fluctuations in vital signs and behavioural responses of brain surgery patients in the intensive care unit: Are they valid indicators of pain? J Adv Nurs 2014; 70:2562-2576

71. Payen JF, Bru O, Bosson JL, et al: Assessing pain in critically ill sedated patients by using a Behavioral Pain Scale. Crit Care Med $2001 ; 29: 2258-2263$

72. Siffleet J, Young J, Nikoletti S, et al: Patients' self-report of procedural pain in the intensive care unit. J Clin Nurs 2007; 16:2142-2148

73. Young J, Siffleet J, Nikoletti S, et al: Use of a Behavioural Pain Scale to assess pain in ventilated, unconscious and/or sedated patients. Intensive Crit Care Nurs 2006; 22:32-39

74. Arbour $\mathrm{C}$, Choinière $\mathrm{M}$, Topolovec-Vranic J, et al: Can fluctuations in vital signs be used for pain assessment in critically ill patients with a traumatic brain injury? Pain Res Treat 2014; 2014:175794

75. Hadjistavropoulos T, Craig KD: A theoretical framework for understanding self-report and observational measures of pain: A communications model. Behav Res Ther 2002; 40:551-570

76. Broucqsault-Dédrie C, De Jonckheere J, Jeanne M, et al: Measurement of heart rate variability to assess pain in sedated critically ill patients: A prospective observational study. PLoS One 2016; 11:e0147720
77. Chanques G, Tarri T, Ride A, et al: Analgesia nociception index for the assessment of pain in critically ill patients: A diagnostic accuracy study. Br J Anaesth 2017; 119:812-820

78. Ben-Israel N, Kliger M, Zuckerman G, et al: Monitoring the nociception level: A multi-parameter approach. J Clin Monit Comput 2013; 27:659-668

79. Li D, Miaskowski C, Burkhardt D, et al: Evaluations of physiologic reactivity and reflexive behaviors during noxious procedures in sedated critically ill patients. J Crit Care 2009; 24:472.e9-472.e13

80. Lukaszewicz AC, Dereu D, Gayat E, et al: The relevance of pupillometry for evaluation of analgesia before noxious procedures in the intensive care unit. Anesth Analg 2015; 120:1297-1300

81. Paulus J, Roquilly $\mathrm{A}$, Beloeil $\mathrm{H}$, et al: Pupillary reflex measurement predicts insufficient analgesia before endotracheal suctioning in critically ill patients. Crit Care 2013; 17:R161

82. White PF, Kehlet H, Neal JM, et al; Fast-Track Surgery Study Group: The role of the anesthesiologist in fast-track surgery: From multimodal analgesia to perioperative medical care. Anesth Analg 2007; 104:1380-1396

83. Cattabriga I, Pacini D, Lamazza G, et al: Intravenous paracetamol as adjunctive treatment for postoperative pain after cardiac surgery: A double blind randomized controlled trial. Eur J Cardiothorac Surg 2007; 32:527-531

84. Memis D, Inal MT, Kavalci G, et al: Intravenous paracetamol reduced the use of opioids, extubation time, and opioid-related adverse effects after major surgery in intensive care unit. J Crit Care 2010; 25:458462

85. Cantais A, Schnell D, Vincent F, et al: Acetaminophen-induced changes in systemic blood pressure in critically ill patients: Results of a multicenter cohort study. Crit Care Med 2016; 44:2192-2198

86. Beloeil H, Delage N, Nègre I, et al: The median effective dose of nefopam and morphine administered intravenously for postoperative pain after minor surgery: A prospective randomized double-blinded isobolographic study of their analgesic action. Anesth Analg 2004; 98:395-400

87. Payen JF, Genty C, Mimoz O, et al: Prescribing nonopioids in mechanically ventilated critically ill patients. J Crit Care 2013; 28:534.e7534.12

88. Kim K, Kim WJ, Choi DK, et al: The analgesic efficacy and safety of nefopam in patient-controlled analgesia after cardiac surgery: A randomized, double-blind, prospective study. J Int Med Res 2014; 42:684-692

89. Chanques G, Sebbane M, Constantin JM, et al: Analgesic efficacy and haemodynamic effects of nefopam in critically ill patients. $\mathrm{Br} J$ Anaesth 2011; 106:336-343

90. Corbonnois G, lohom G, Lazarescu C, et al: Unilateral permanent loss of vision after nefopam administration. Ann Fr Anesth Reanim 2013; 32:e113-e115

91. Durrieu G, Olivier P, Bagheri H, et al; French Network of Pharmacovigilance Centers: Overview of adverse reactions to nefopam: An analysis of the French Pharmacovigilance database. Fundam Clin Pharmacol 2007; 21:555-558

92. Godier A, Babinet A, el Metaoua S, et al: A new cause of postoperative confusion syndrome: Nefopam. Ann Fr Anesth Reanim 2002; 21:538-539

93. Assouline B, Tramèr MR, Kreienbühl L, et al: Benefit and harm of adding ketamine to an opioid in a patient-controlled analgesia device for the control of postoperative pain: Systematic review and meta-analyses of randomized controlled trials with trial sequential analyses. Pain 2016; 157:2854-2864

94. Wang L, Johnston B, Kaushal A, et al: Ketamine added to morphine or hydromorphone patient-controlled analgesia for acute postoperative pain in adults: A systematic review and meta-analysis of randomized trials. Can J Anaesth 2016; 63:311-325

95. Guillou N, Tanguy M, Seguin P, et al: The effects of small-dose ketamine on morphine consumption in surgical intensive care unit patients after major abdominal surgery. Anesth Analg 2003; 97:843-847

96. Pandey CK, Bose N, Garg G, et al: Gabapentin for the treatment of pain in Guillain-Barré syndrome: A double-blinded, placebo-controlled, crossover study. Anesth Analg 2002; 95:1719-1723 
97. Pandey CK, Raza M, Tripathi M, et al: The comparative evaluation of gabapentin and carbamazepine for pain management in GuillainBarré syndrome patients in the intensive care unit. Anesth Analg 2005; 101:220-225

98. Pesonen A, Suojaranta-Ylinen R, Hammarén E, et al: Pregabalin has an opioid-sparing effect in elderly patients after cardiac surgery: A randomized placebo-controlled trial. $\mathrm{Br} J$ Anaesth 2011; 106:873-881

99. Joshi SS, Jagadeesh AM: Efficacy of perioperative pregabalin in acute and chronic post-operative pain after off-pump coronary artery bypass surgery: A randomized, double-blind placebo controlled trial. Ann Card Anaesth 2013; 16:180-185

100. Insler SR, O'Connor M, Samonte AF, et al: Lidocaine and the inhibition of postoperative pain in coronary artery bypass patients. $J$ Cardiothorac Vasc Anesth 1995; 9:541-546

101. Kranke P, Jokinen J, Pace NL, et al: Continuous intravenous perioperative lidocaine infusion for postoperative pain and recovery. Cochrane Database Syst Rev 2015:CD009642

102. Hynninen MS, Cheng DC, Hossain I, et al: Non-steroidal anti-inflammatory drugs in treatment of postoperative pain after cardiac surgery. Can J Anaesth 2000; 47:1182-1187

103. Oberhofer D, Skok J, Nesek-Adam V: Intravenous ketoprofen in postoperative pain treatment after major abdominal surgery. World $J$ Surg 2005; 29:446-449

104. Wick EC, Grant MC, Wu CL: Postoperative multimodal analgesia pain management with nonopioid analgesics and techniques: A review. JAMA Surg 2017; 152:691-697

105. Soriano SG: Neurotoxicity of ketamine: Known unknowns. Crit Care Med 2012; 40:2518-2519

106. Payen JF, Bosson JL, Chanques G, et al; DOLOREA Investigators: Pain assessment is associated with decreased duration of mechanical ventilation in the intensive care unit: A post hoc analysis of the DOLOREA study. Anesthesiology 2009; 111:1308-1316

107. Brook $A D$, Ahrens TS, Schaiff $R$, et al: Effect of a nursing-implemented sedation protocol on the duration of mechanical ventilation. Crit Care Med 1999; 27:2609-2615

108. Quenot JP, Ladoire S, Devoucoux F, et al: Effect of a nurse-implemented sedation protocol on the incidence of ventilator-associated pneumonia. Crit Care Med 2007; 35:2031-2036

109. Robinson BR, Mueller EW, Henson K, et al: An analgesia-deliriumsedation protocol for critically ill trauma patients reduces ventilator days and hospital length of stay. J Trauma 2008; 65:517-526

110. Chanques G, Jaber S, Barbotte E, et al: Impact of systematic evaluation of pain and agitation in an intensive care unit. Crit Care Med 2006; 34:1691-1699

111. Marshall J, Finn CA, Theodore AC: Impact of a clinical pharmacist-enforced intensive care unit sedation protocol on duration of mechanical ventilation and hospital stay. Crit Care Med 2008; 36:427-433

112. Kress JP, Pohlman AS, O'Connor MF, et al: Daily interruption of sedative infusions in critically ill patients undergoing mechanical ventilation. N Engl J Med 2000; 342:1471-1477

113. Broscious SK: Music: An intervention for pain during chest tube removal after open heart surgery. Am J Crit Care 1999; 8:410-415

114. Chan MF: Effects of music on patients undergoing a C-clamp procedure after percutaneous coronary interventions: A randomized controlled trial. Heart Lung 2007; 36:431-439

115. Cooke M, Chaboyer W, Schluter $P$, et al: The effect of music on discomfort experienced by intensive care unit patients during turning: A randomized cross-over study. Int J Nurs Pract 2010; 16:125-131

116. Chiasson AM, Linda Baldwin A, McLaughlin C, et al: The effect of live spontaneous harp music on patients in the intensive care unit. Evid Based Complement Alternat Med 2013; 2013:428731

117. Kshettry VR, Carole LF, Henly SJ, et al: Complementary alternative medical therapies for heart surgery patients: Feasibility, safety, and impact. Ann Thorac Surg 2006; 81:201-205

118. Casey E, Lane A, Kuriakose D, et al: Bolus remifentanil for chest drain removal in ICU: A randomized double-blind comparison of three modes of analgesia in post-cardiac surgical patients. Intensive Care Med 2010; 36:1380-1385
119. Ahlers SJ, van Gulik L, van Dongen EP, et al: Efficacy of an intravenous bolus of morphine 2.5 versus morphine $7.5 \mathrm{mg}$ for procedural pain relief in postoperative cardiothoracic patients in the intensive care unit: A randomised double-blind controlled trial. Anaesth Intensive Care 2012; 40:417-426

120. Robleda G, Roche-Campo F, Sendra MÀ, et al: Fentanyl as pre-emptive treatment of pain associated with turning mechanically ventilated patients: A randomized controlled feasibility study. Intensive Care Med 2016; 42:183-191

121. Akrofi M, Miller S, Colfar S, et al: A randomized comparison of three methods of analgesia for chest drain removal in postcardiac surgical patients. Anesth Analg 2005; 100:205-209

122. Van Allen NR, Krafft PR, Leitzke AS, et al: The role of volatile anesthetics in cardioprotection: A systematic review. Med Gas Res 2012; 2:22

123. Bryden FM, McFarlane $H$, Tunstall ME, et al: Isoflurane for removal of chest drains after cardiac surgery. Anaesthesia 1997; 52:173-175

124. Puntillo K, Ley SJ: Appropriately timed analgesics control pain due to chest tube removal. Am J Crit Care 2004; 13:292-301; discussion 302; quiz 303

125. Singh $M$, Gopinath R: Topical analgesia for chest tube removal in cardiac patients. J Cardiothorac Vasc Anesth 2005; 19:719-722

126. Mosso-Vázquez JL, Gao K, Wiederhold BK, et al: Virtual reality for pain management in cardiac surgery. Cyberpsychol Behav Soc Netw 2014; 17:371-378

127. Berger MM, Davadant M, Marin C, et al: Impact of a pain protocol including hypnosis in major burns. Burns 2010; 36:639-646

128. Asadizaker M, Fathizadeh A, Haidari A, et al: The effect of foot and hand massage on postoperative cardiac surgery pain. Indian J NuCl Med 2011; 3:165-169

129. Piotrowski MM, Paterson C, Mitchinson A, et al: Massage as adjuvant therapy in the management of acute postoperative pain: A preliminary study in men. J Am Coll Surg 2003; 197:1037-1046

130. Mitchinson AR, Kim HM, Rosenberg JM, et al: Acute postoperative pain management using massage as an adjuvant therapy: A randomized trial. Arch Surg 2007; 142:1158-1167

131. Jaber $S$, Bahloul H, Guétin $S$, et al: Effects of music therapy in intensive care unit without sedation in weaning patients versus non-ventilated patients. Ann Fr Anesth Reanim 2007; 26:30-38

132. Özer N, Karaman Özlü Z, Arslan S, et al: Effect of music on postoperative pain and physiologic parameters of patients after open heart surgery. Pain Manag Nurs 2013; 14:20-28

133. Chlan LL, Weinert CR, Heiderscheit A, et al: Effects of patientdirected music intervention on anxiety and sedative exposure in critically ill patients receiving mechanical ventilatory support: A randomized clinical trial. JAMA 2013; 309:2335-2344

134. Gorji HM, Nesami BM, Ayyasi M, et al: Comparison of ice packs application and relaxation therapy in pain reduction during chest tube removal following cardiac surgery. N Am J Med Sci 2014; 6:19-24

135. Sauls J: The use of ice for pain associated with chest tube removal. Pain Manag Nurs 2002; 3:44-52

136. Cepeda MS, Africano JM, Polo R, et al: What decline in pain intensity is meaningful to patients with acute pain? Pain 2003; 105:151-157

137. Houston S, Jesurum J: The quick relaxation technique: Effect on pain associated with chest tube removal. Appl Nurs Res 1999; 12:196-205

138. Friesner SA, Curry DM, Moddeman GR: Comparison of two painmanagement strategies during chest tube removal: Relaxation exercise with opioids and opioids alone. Heart Lung 2006; 35:269-276

139. Aday AW, Dell'orfano H, Hirning BA, et al: Evaluation of a clinical pathway for sedation and analgesia of mechanically ventilated patients in a cardiac intensive care unit (CICU): The Brigham and Women's Hospital Levine CICU sedation pathways. Eur Heart $J$ Acute Cardiovasc Care 2013; 2:299-305

140. Awissi DK, Bégin C, Moisan J, et al: I-SAVE study: Impact of sedation, analgesia, and delirium protocols evaluated in the intensive care unit: An economic evaluation. Ann Pharmacother 2012; 46:21-28

141. Dale CR, Bryson CL, Fan VS, et al: A greater analgesia, sedation, delirium order set quality score is associated with a decreased 
duration of mechanical ventilation in cardiovascular surgery patients. Crit Care Med 2013; 41:2610-2617

142. Degrado JR, Anger KE, Szumita PM, et al: Evaluation of a local ICU sedation guideline on goal-directed administration of sedatives and analgesics. J Pain Res 2011; 4:127-134

143. Diby M, Romand JA, Frick S, et al: Reducing pain in patients undergoing cardiac surgery after implementation of a quality improvement postoperative pain treatment program. J Crit Care 2008; 23:359-371

144. Egerod I, Jensen MB, Herling SF, et al: Effect of an analgo-sedation protocol for neurointensive patients: A two-phase interventional nonrandomized pilot study. Crit Care 2010; 14:R71

145. Erdek MA, Pronovost PJ: Improving assessment and treatment of pain in the critically ill. Int J Qual Health Care 2004; 16:59-64

146. Faust AC, Rajan $P$, Sheperd LA, et al: Impact of an analgesia-based sedation protocol on mechanically ventilated patients in a medical intensive care unit. Anesth Analg 2016; 123:903-909

147. MacLaren R, Plamondon JM, Ramsay KB, et al: A prospective evaluation of empiric versus protocol-based sedation and analgesia. Pharmacotherapy 2000; 20:662-672

148. Park G, Lane M, Rogers S, et al: A comparison of hypnotic and analgesic based sedation in a general intensive care unit. $\mathrm{Br} J$ Anaesth 2007; 98:76-82

149. Sneyers B, Laterre PF, Perreault MM, et al: Current practices and barriers impairing physicians' and nurses' adherence to analgosedation recommendations in the intensive care unit-a national survey. Crit Care 2014; 18:655

150. Tedders KM, McNorton KN, Edwin SB: Efficacy and safety of analgosedation with fentanyl compared with traditional sedation with propofol. Pharmacotherapy 2014; 34:643-647

151. van Gulik L, Ahlers SJ, Brkić Z, et al: Improved analgesia after the realisation of a pain management programme in ICU patients after cardiac surgery. Eur J Anaesthesiol 2010; 27:900-905

152. van Valen $R$, van Vuuren $H$, van Domburg $R T$, et al: Pain management after cardiac surgery: Experience with a nurse-driven pain protocol. Eur J Cardiovasc Nurs 2012; 11:62-69

153. Breen D, Karabinis A, Malbrain M, et al: Decreased duration of mechanical ventilation when comparing analgesia-based sedation using remifentanil with standard hypnotic-based sedation for up to 10 days in intensive care unit patients: $A$ randomised trial [ISRCTN47583497]. Crit Care 2005; 9:R200-R210

154. Karabinis A, Mandragos K, Stergiopoulos S, et al: Safety and efficacy of analgesia-based sedation with remifentanil versus standard hypnotic-based regimens in intensive care unit patients with brain injuries: A randomised, controlled trial [ISRCTN50308308]. Crit Care 2004; 8:R268-R280

155. Rozendaal FW, Spronk PE, Snellen FF, et al; UltiSAFE Investigators: Remifentanil-propofol analgo-sedation shortens duration of ventilation and length of ICU stay compared to a conventional regimen: $A$ centre randomised, cross-over, open-label study in the Netherlands. Intensive Care Med 2009; 35:291-298

156. Strøm T, Martinussen T, Toft P: A protocol of no sedation for critically ill patients receiving mechanical ventilation: A randomised trial. Lancet 2010; 375:475-480

157. Kollef MH, Levy NT, Ahrens TS, et al: The use of continuous i.v. sedation is associated with prolongation of mechanical ventilation. Chest 1998; $114: 541-548$

158. Pandharipande $P$, Shintani A, Peterson J, et al: Lorazepam is an independent risk factor for transitioning to delirium in intensive care unit patients. Anesthesiology 2006; 104:21-26

159. Brattebø G, Hofoss D, Flaatten H, et al: Effect of a scoring system and protocol for sedation on duration of patients' need for ventilator support in a surgical intensive care unit. BMJ 2002; 324:1386-1389

160. Sessler CN, Gosnell MS, Grap MJ, et al: The Richmond AgitationSedation Scale: Validity and reliability in adult intensive care unit patients. Am J Respir Crit Care Med 2002; 166:1338-1344

161. Riker RR, Fraser GL, Simmons LE, et al: Validating the SedationAgitation Scale with the Bispectral Index and Visual Analog Scale in adult ICU patients after cardiac surgery. Intensive Care Med 2001; 27:853-858
162. Roberts DJ, Haroon B, Hall RI: Sedation for critically ill or injured adults in the intensive care unit: A shifting paradigm. Drugs 2012; 72:1881-1916

163. MacKenzie M, Hall R: Pharmacogenomics and pharmacogenetics for the intensive care unit: A narrative review. Can J Anaesth 2017; 64:45-64

164. Treggiari MM, Romand JA, Yanez ND, et al: Randomized trial of light versus deep sedation on mental health after critical illness. Crit Care Med 2009; 37:2527-2534

165. Girard TD, Kress JP, Fuchs BD, et al: Efficacy and safety of a paired sedation and ventilator weaning protocol for mechanically ventilated patients in intensive care (Awakening and Breathing Controlled trial): A randomised controlled trial. Lancet 2008; 371:126-134

166. Shehabi $Y$, Bellomo $R$, Reade MC, et al; Sedation Practice in Intensive Care Evaluation (SPICE) Study Investigators; ANZICS Clinical Trials Group: Early intensive care sedation predicts longterm mortality in ventilated critically ill patients. Am J Respir Crit Care Med 2012; 186:724-731

167. Riker RR, Shehabi Y, Bokesch PM, et al; SEDCOM (Safety and Efficacy of Dexmedetomidine Compared With Midazolam) Study Group: Dexmedetomidine vs midazolam for sedation of critically ill patients: A randomized trial. JAMA 2009; 301:489-499

168. Shehabi $\mathrm{Y}$, Bellomo R, Reade MC, et al; Sedation Practice in Intensive Care Evaluation Study Investigators; Australian and New Zealand Intensive Care Society Clinical Trials Group: Early goal-directed sedation versus standard sedation in mechanically ventilated critically ill patients: A pilot study. Crit Care Med 2013; 41:1983-1991

169. Bugedo G, Tobar E, Aguirre M, et al: The implementation of an analgesia-based sedation protocol reduced deep sedation and proved to be safe and feasible in patients on mechanical ventilation. Rev Bras Ter Intensiva 2013; 25:188-196

170. Treggiari M: Randomized trial of light versus deep sedation on mental health after critical illness. Crit Care Med 2010; 38:349-350

171. Tanaka LM, Azevedo LC, Park M, et al; ERICC Study Investigators: Early sedation and clinical outcomes of mechanically ventilated patients: A prospective multicenter cohort study. Crit Care 2014; 18:R156

172. Pandharipande PP, Pun BT, Herr DL, et al: Effect of sedation with dexmedetomidine vs lorazepam on acute brain dysfunction in mechanically ventilated patients: The MENDS randomized controlled trial. JAMA 2007; 298:2644-2653

173. Muller L, Chanques G, Bourgaux C, et al: Impact of the use of propofol remifentanil goal-directed sedation adapted by nurses on the time to extubation in mechanically ventilated ICU patients: The experience of a French ICU. Ann Fr Anesth Reanim 2008; 27:481.e1-481.e8

174. Samuelson KA, Lundberg D, Fridlund B: Light vs. heavy sedation during mechanical ventilation after oesophagectomy-a pilot experimental study focusing on memory. Acta Anaesthesiol Scand 2008; 52:1116-1123

175. Shehabi Y, Chan L, Kadiman S, et al; Sedation Practice in Intensive Care Evaluation (SPICE) Study Group Investigators: Sedation depth and long-term mortality in mechanically ventilated critically ill adults: A prospective longitudinal multicentre cohort study. Intensive Care Med 2013; 39:910-918

176. Balzer F, Weiß B, Kumpf O, et al: Early deep sedation is associated with decreased in-hospital and two-year follow-up survival. Crit Care 2015; 19:197

177. Shehabi Y, Bellomo R, Kadiman S, et al; Sedation Practice in Intensive Care Evaluation (SPICE) Study Investigators and the Australian and New Zealand Intensive Care Society Clinical Trials Group: Sedation intensity in the first 48 hours of mechanical ventilation and 180-day mortality: A multinational prospective longitudinal cohort study. Crit Care Med 2018; 46:850-859

178. Carson SS, Kress JP, Rodgers JE, et al: A randomized trial of intermittent lorazepam versus propofol with daily interruption in mechanically ventilated patients. Crit Care Med 2006; 34:1326-1332

179. Mehta S, Burry L, Cook D, et al; SLEAP Investigators; Canadian Critical Care Trials Group: Daily sedation interruption in mechanically ventilated critically ill patients cared for with a sedation protocol: A randomized controlled trial. JAMA 2012; 308:1985-1992 
180. de Wit M, Gennings C, Jenvey WI, et al: Randomized trial comparing daily interruption of sedation and nursing-implemented sedation algorithm in medical intensive care unit patients. Crit Care 2008; 12:R70

181. Nassar Junior AP, Park M: Daily sedative interruption versus intermittent sedation in mechanically ventilated critically ill patients: A randomized trial. Ann Intensive Care 2014; 4:14

182. Yiliaz C, Kelebek Girgin N, Ozdemir N, et al: The effect of nursingimplemented sedation on the duration of mechanical ventilation in the ICU. Ulus Travma Acil Cerrahi Derg 2010; 16:521-526

183. Grounds RM, Lalor JM, Lumley J, et al: Propofol infusion for sedation in the intensive care unit: Preliminary report. Br Med J (Clin Res Ed) $1987 ; 294: 397-400$

184. McMurray TJ, Collier PS, Carson IW, et al: Propofol sedation after open heart surgery. A clinical and pharmacokinetic study. Anaesthesia 1990; 45:322-326

185. Snellen F, Lauwers P, Demeyere R, et al: The use of midazolam versus propofol for short-term sedation following coronary artery bypass grafting. Intensive Care Med 1990; 16:312-316

186. Roekaerts PM, Huygen FJ, de Lange S: Infusion of propofol versus midazolam for sedation in the intensive care unit following coronary artery surgery. J Cardiothorac Vasc Anesth 1993; 7:142-147

187. Searle NR, Côté S, Taillefer J, et al: Propofol or midazolam for sedation and early extubation following cardiac surgery. Can J Anaesth 1997; 44:629-635

188. Dowd NP, Karski JM, Cheng DC, et al: Fast-track cardiac anaesthesia in the elderly: Effect of two different anaesthetic techniques on mental recovery. Br J Anaesth $2001 ; 86: 68-76$

189. Huey-Ling L, Chun-Che S, Jen-Jen T, et al: Comparison of the effect of protocol-directed sedation with propofol vs. midazolam by nurses in intensive care: Efficacy, haemodynamic stability and patient satisfaction. J Clin Nurs 2008; 17:1510-1517

190. Oliver WC Jr, Nuttall GA, Murari T, et al: A prospective, randomized, double-blind trial of 3 regimens for sedation and analgesia after cardiac surgery. J Cardiothorac Vasc Anesth 2011; 25:110-119

191. Carrasco G, Molina R, Costa J, et al: Propofol vs midazolam in short-, medium-, and long-term sedation of critically ill patients. A cost-benefit analysis. Chest 1993; 103:557-564

192. Chamorro C, de Latorre FJ, Montero A, et al: Comparative study of propofol versus midazolam in the sedation of critically ill patients: Results of a prospective, randomized, multicenter trial. Crit Care Med 1996; 24:932-939

193. Weinbroum AA, Halpern P, Rudick V, et al: Midazolam versus propofol for long-term sedation in the ICU: A randomized prospective comparison. Intensive Care Med 1997; 23:1258-1263

194. Sanchez-Izquierdo-Riera JA, Caballero-Cubedo RE, Perez-Vela JL, et al: Propofol versus midazolam: Safety and efficacy for sedating the severe trauma patient. Anesth Analg 1998; 86:1219-1224

195. Sandiumenge Camps A, Sanchez-lzquierdo Riera JA, Toral Vazquez $\mathrm{D}$, et al: Midazolam and $2 \%$ propofol in long-term sedation of traumatized critically ill patients: Efficacy and safety comparison. Crit Care Med 2000; 28:3612-3619

196. Mesnil M, Capdevila X, Bringuier S, et al: Long-term sedation in intensive care unit: A randomized comparison between inhaled sevoflurane and intravenous propofol or midazolam. Intensive Care Med $2011 ; 37: 933-941$

197. Zhou Y, Jin X, Kang Y, et al: Midazolam and propofol used alone or sequentially for long-term sedation in critically ill, mechanically ventilated patients: A prospective, randomized study. Crit Care 2014; 18:R1 22

198. Boeke A, Lauwers J, Schurink G: A pilot study to compare the use of propofol and midazolam for long-term sedation. J Drug Dev 1989; 2:71-72

199. Barrientos-Vega R, Mar Sánchez-Soria M, Morales-García C, et al: Prolonged sedation of critically ill patients with midazolam or propofol: Impact on weaning and costs. Crit Care Med 1997; 25:33-40

200. Costa J, Cabré L, Molina R, et al: Cost of ICU sedation: Comparison of empirical and controlled sedation methods. Clin Intensive Care $1994 ; 5: 17-21$

201. Hall RI, Sandham D, Cardinal P, et al; Study Investigators: Propofol vs midazolam for ICU sedation: A Canadian multicenter randomized trial. Chest 2001; 119:1151-1159
202. Srivastava VK, Agrawal S, Kumar S, et al: Comparison of dexmedetomidine, propofol and midazolam for short-term sedation in postoperatively mechanically ventilated neurosurgical patients. J Clin Diagn Res 2014; 8:GC04-GC07

203. Jakob SM, Ruokonen E, Grounds RM, et al; Dexmedetomidine for Long-Term Sedation Investigators: Dexmedetomidine vs midazolam or propofol for sedation during prolonged mechanical ventilation: Two randomized controlled trials. JAMA 2012; 307:1151-1160

204. MacLaren R, Preslaski CR, Mueller SW, et al: A randomized, doubleblind pilot study of dexmedetomidine versus midazolam for intensive care unit sedation: Patient recall of their experiences and short-term psychological outcomes. J Intensive Care Med 2015; 30:167-175

205. Xu JB, Wang YZ, Shi QS: A combined protocol for dexmedetomidine used in prolonged sedation in intensive care unit. Mod Med J Chin 2012; 14:20-22

206. Herr DL, Sum-Ping ST, England M: ICU sedation after coronary artery bypass graft surgery: Dexmedetomidine-based versus propofol-based sedation regimens. J Cardiothorac Vasc Anesth 2003; 17:576-584

207. Turnbull AE, Sepulveda KA, Dinglas VD, et al: Core domains for clinical research in acute respiratory failure survivors: An international modified Delphi consensus study. Crit Care Med 2017; 45:1001-1010

208. Olson DM, Thoyre SM, Peterson ED, et al: A randomized evaluation of bispectral index-augmented sedation assessment in neurological patients. Neurocrit Care 2009; 11:20-27

209. Yang KS, Habib AS, Lu M, et al: A prospective evaluation of the incidence of adverse events in nurse-administered moderate sedation guided by sedation scores or bispectral index. Anesth Analg 2014; 119:43-48

210. Mahmood S, Parchani A, El-Menyar A, et al: Utility of bispectral index in the management of multiple trauma patients. Surg Neurol Int 2014; 5:141

211. Fraser GL, Riker RR: Bispectral index monitoring in the intensive care unit provides more signal than noise. Pharmacotherapy 2005; 25:19S-27S

212. De Deyne C, Struys M, Decruyenaere J, et al: Use of continuous bispectral EEG monitoring to assess depth of sedation in ICU patients. Intensive Care Med 1998; 24:1294-1298

213. Simmons LE, Riker RR, Prato BS, et al: Assessing sedation during intensive care unit mechanical ventilation with the Bispectral Index and the Sedation-Agitation Scale. Crit Care Med 1999; 27:1499-1504

214. Walder B, Suter PM, Romand JA: Evaluation of two processed EEG analyzers for assessment of sedation after coronary artery bypass grafting. Intensive Care Med 2001; 27:107-114

215. Frenzel D, Greim CA, Sommer C, et al: Is the bispectral index appropriate for monitoring the sedation level of mechanically ventilated surgical ICU patients? Intensive Care Med 2002; 28:178-183

216. Mondello E, Siliotti R, Noto G, et al: Bispectral index in ICU: Correlation with Ramsay Score on assessment of sedation level. $J$ Clin Monit Comput 2002; 17:271-277

217. Nasraway SA SA Jr, Wu EC, Kelleher RM, et al: How reliable is the bispectral index in critically ill patients? A prospective, comparative, single-blinded observer study. Crit Care Med 2002; 30:1483-1487

218. Riess ML, Graefe UA, Goeters C, et al: Sedation assessment in critically ill patients with bispectral index. Eur J Anaesthesiol 2002; 19:18-22

219. de Wit M, Epstein SK: Administration of sedatives and level of sedation: Comparative evaluation via the Sedation-Agitation Scale and the Bispectral Index. Am J Crit Care 2003; 12:343-348

220. Ely EW, Truman B, Shintani A, et al: Monitoring sedation status over time in ICU patients: Reliability and validity of the Richmond Agitation-Sedation Scale (RASS). JAMA 2003; 289:2983-2991

221. Doi M, Morita $\mathrm{K}$, Mantzaridis $\mathrm{H}$, et al: Prediction of responses to various stimuli during sedation: A comparison of three EEG variables. Intensive Care Med 2005; 31:41-47

222. Tonner $\mathrm{PH}$, Wei $\mathrm{C}$, Bein $\mathrm{B}$, et al: Comparison of two bispectral index algorithms in monitoring sedation in postoperative intensive care patients. Crit Care Med 2005; 33:580-584 
223. Chisholm CJ, Zurica J, Mironov D, et al: Comparison of electrophysiologic monitors with clinical assessment of level of sedation. Mayo Clin Proc 2006; 81:46-52

224. Consales G, Chelazzi C, Rinaldi S, et al: Bispectral Index compared to Ramsay score for sedation monitoring in intensive care units. Minerva Anestesio/ 2006; 72:329-336

225. Turkmen A, Altan A, Turgut N, et al: The correlation between the Richmond Agitation-Sedation Scale and bispectral index during dexmedetomidine sedation. Eur J Anaesthesiol 2006; 23:300-304

226. Hernández-Gancedo $\mathrm{C}$, Pestaña $\mathrm{D}$, Pérez-Chrzanowska $\mathrm{H}$, et al: Comparing entropy and the bispectral index with the Ramsay score in sedated ICU patients. J Clin Monit Comput 2007; 21:295-302

227. Sackey PV, Radell PJ, Granath F, et al: Bispectral index as a predictor of sedation depth during isoflurane or midazolam sedation in ICU patients. Anaesth Intensive Care 2007; 35:348-356

228. Haenggi M, Ypparila-Wolters H, Bieri C, et al: Entropy and bispectral index for assessment of sedation, analgesia and the effects of unpleasant stimuli in critically ill patients: An observational study. Crit Care 2008; 12:R119

229. Lu CH, Ou-Yang HY, Man KM, et al: Relative reliability of the auditory evoked potential and bispectral index for monitoring sedation level in surgical intensive care patients. Anaesth Intensive Care 2008; 36:553-559

230. Arbour R, Waterhouse J, Seckel MA, et al: Correlation between the Sedation-Agitation Scale and the Bispectral Index in ventilated patients in the intensive care unit. Heart Lung 2009; 38:336-345

231. Trouiller $P$, Fangio $P$, Paugam-Burtz $C$, et al: Frequency and clinical impact of preserved bispectral index activity during deep sedation in mechanically ventilated ICU patients. Intensive Care Med 2009; 35:2096-2104

232. Karamchandani K, Rewari V, Trikha A, et al: Bispectral index correlates well with Richmond Agitation Sedation Scale in mechanically ventilated critically ill patients. J Anesth 2010; 24:394-398

233. Plaschke K, Fichtenkamm P, Schramm C, et al: Early postoperative delirium after open-heart cardiac surgery is associated with decreased bispectral EEG and increased cortisol and interleukin-6. Intensive Care Med 2010; 36:2081-2089

234. Ogilvie MP, Pereira BM, Ryan ML, et al: Bispectral index to monitor propofol sedation in trauma patients. J Trauma 2011; 71:1415-1421

235. LeBlanc JM, Dasta JF, Pruchnicki MC, et al: Bispectral index values, sedation-agitation scores, and plasma lorazepam concentrations in critically ill surgical patients. Am J Crit Care 2012; 21:99-105

236. Yaman F, Ozcan N, Ozcan A, et al: Assesment of correlation between bispectral index and four common sedation scales used in mechanically ventilated patients in ICU. Eur Rev Med Pharmacol Sci 2012; 16:660-666

237. Paliwal B, Rai P, Kamal M, et al: Comparison between dexmedetomidine and propofol with validation of bispectral index for sedation in mechanically ventilated intensive care patients. J Clin Diagn Res 2015; 9:UC01-UC05

238. Prottengeier J, Moritz A, Heinrich S, et al: Sedation assessment in a mobile intensive care unit: $A$ prospective pilot-study on the relation of clinical sedation scales and the bispectral index. Crit Care 2014; 18:615

239. Wang $\mathrm{ZH}$, Chen $\mathrm{H}$, Yang $\mathrm{YL}$, et al: Bispectral index can reliably detect deep sedation in mechanically ventilated patients: A prospective multicenter validation study. Anesth Analg 2017; 125:176-183

240. Department of Health and Human Services, Centers for Medicare \& Medicaid Services: 42 CFR Part 482 Medicare and Medicaid Programs; Hospital Conditions of Participation: Patients' Rights; Final Rule. Available at: https://www.cms.gov/RegulationsandGuidance/Legislation/CFCsAndCoPs/Hospitals.html. Accessed August 17, 2017

241. Elliott D, Aitken LM, Bucknall TK, et al; Australian and New Zealand Intensive Care Society Clinical Trials Group; George Institute for Global Health: Patient comfort in the intensive care unit: A multicentre, binational point prevalence study of analgesia, sedation and delirium management. Crit Care Resusc 2013; 15:213-219

242. Benbenbishty J, Adam S, Endacott R: Physical restraint use in intensive care units across Europe: The PRICE study. Intensive Crit Care Nurs 2010; 26:241-245
243. Burk RS, Grap MJ, Munro CL, et al: Predictors of agitation in critically ill adults. Am J Crit Care 2014; 23:414-423

244. Burry LD, Williamson DR, Perreault MM, et al: Analgesic, sedative, antipsychotic, and neuromuscular blocker use in Canadian intensive care units: A prospective, multicentre, observational study. Can J Anaesth 2014; 61:619-630

245. Chang LY, Wang KW, Chao YF: Influence of physical restraint on unplanned extubation of adult intensive care patients: A case-control study. Am J Crit Care 2008; 17:408-415; quiz 416

246. Choi E, Song M: Physical restraint use in a Korean ICU. J Clin Nurs 2003; 12:651-659

247. Curry K, Cobb S, Kutash M, et al: Characteristics associated with unplanned extubations in a surgical intensive care unit. $A m$ J Crit Care 2008; 17:45-51; quiz 52

248. Happ MB, Roesch T, Kagan SH: Communication needs, methods, and perceived voice quality following head and neck surgery: A literature review. Cancer Nurs 2004; 27:1-9

249. Kandeel NA, Attia AK: Physical restraints practice in adult intensive care units in Egypt. Nurs Health Sci 2013; 15:79-85

250. Krüger $C$, Mayer $H$, Haastert $B$, et al: Use of physical restraints in acute hospitals in Germany: A multi-centre cross-sectional study. Int J Nurs Stud 2013; 50:1599-1606

251. Kwizera A, Nakibuuka J, Ssemogerere L, et al: Incidence and risk factors for delirium among mechanically ventilated patients in an African intensive care setting: An observational multicenter study. Crit Care Res Pract 2015; 2015:491780

252. Langley G, Schmollgruber S, Egan A: Restraints in intensive care units-a mixed method study. Intensive Crit Care Nurs 2011; 27:67-75

253. Liu JJ, Chou FH, Yeh SH: Basic needs and their predictors for intubated patients in surgical intensive care units. Heart Lung 2009; 38:208-216

254. Lucidarme O, Seguin A, Daubin C, et al: Nicotine withdrawal and agitation in ventilated critically ill patients. Crit Care 2010; 14:R58

255. Martin B, Mathisen L: Use of physical restraints in adult critical care: A bicultural study. Am J Crit Care 2005; 14:133-142

256. Martín Iglesias V, Pontón Soriano C, Quintián Guerra MT, et al: Mechanical restraint: Its use in intensive cares. Enferm Intensiva 2012; 23:164-170

257. Mehta S, Cook D, Devlin JW, et al; SLEAP Investigators; Canadian Critical Care Trials Group: Prevalence, risk factors, and outcomes of delirium in mechanically ventilated adults. Crit Care Med 2015; 43:557-566

258. Micek ST, Anand NJ, Laible BR, et al: Delirium as detected by the CAM-ICU predicts restraint use among mechanically ventilated medical patients. Crit Care Med 2005; 33:1260-1265

259. Van Rompaey B, Elseviers MM, Schuurmans MJ, et al: Risk factors for delirium in intensive care patients: A prospective cohort study. Crit Care 2009; 13:R77

260. Vance DL: Effect of a treatment interference protocol on clinical decision making for restraint use in the intensive care unit: A pilot study. AACN Clin Issues 2003; 14:82-91

261. van der Kooi AW, Peelen LM, Raijmakers RJ, et al: Use of physical restraints in Dutch intensive care units: A prospective multicenter study. Am J Crit Care 2015; 24:488-495

262. Akansel N: Physical restraint practices among ICU nurses in one university hospital in weastern [sic] turkey. Health Science Journal 2017; $1: 7$

263. Leith BA: Canadian critical care nurses and physical restraints. Off J Can Assoc Crit Care Nurs 1999; 10:10-14

264. Minnick AF, Fogg L, Mion LC, et al: Resource clusters and variation in physical restraint use. J Nurs Scholarsh 2007; 39:363-370

265. Turgay AS, Sari D, Genc RE: Physical restraint use in Turkish intensive care units. Clin Nurse Spec 2009; 23:68-72

266. Yönt GH, Korhan EA, Dizer B, et al: Examination of ethical dilemmas experienced by adult intensive care unit nurses in physical restraint practices. Holist Nurs Pract 2014; 28:85-90 
267. Ismaeil MF, El-Shahat HM, El-Gammal MS, et al: Unplanned versus planned extubation in respiratory intensive care unit, predictors of outcome. Egypt J Chest Dis Tuberc 2014; 63:219-231

268. Rose L, Burry L, Mallick R, et al: Prevalence, risk factors, and outcomes associated with physical restraint use in mechanically ventilated adults. J Crit Care 2016; 31:31-35

269. Minnick A, Leipzig RM, Johnson ME: Elderly patients' reports of physical restraint experiences in intensive care units. Am J Crit Care $2001 ; 10: 168-171$

270. McPherson JA, Wagner CE, Boehm LM, et al: Delirium in the cardiovascular ICU: Exploring modifiable risk factors. Crit Care Med 2013; 41:405-413

271. Yeh $\mathrm{SH}, \mathrm{Hsiao} \mathrm{CY}, \mathrm{Ho} \mathrm{TH}$, et al: The effects of continuing education in restraint reduction on novice nurses in intensive care units. $J$ Nurs Res 2004; 12:246-256

272. Michaud CJ, Thomas WL, McAllen KJ: Early pharmacological treatment of delirium may reduce physical restraint use: A retrospective study. Ann Pharmacother 2014; 48:328-334

273. Titsworth WL, Hester J, Correia T, et al: The effect of increased mobility on morbidity in the neurointensive care unit. J Neurosurg 2012; 116:1379-1388

274. Ely EW, Margolin R, Francis J, et al: Evaluation of delirium in critically ill patients: Validation of the Confusion Assessment Method for the Intensive Care Unit (CAM-ICU). Crit Care Med 2001; 29:1370-1379

275. Bergeron N, Dubois MJ, Dumont M, et al: Intensive Care Delirium Screening Checklist: Evaluation of a new screening tool. Intensive Care Med 2001; 27:859-864

276. Slooter AJ, Van De Leur RR, Zaal IJ: Delirium in critically ill patients. Handb Clin Neurol 2017; 141:449-466

277. Pandharipande PP, Ely EW, Arora RC, et al: The intensive care delirium research agenda: A multinational, interprofessional perspective. Intensive Care Med 2017; 43:1329-1339

278. Zaal IJ, Devlin JW, Peelen LM, et al: A systematic review of risk factors for delirium in the ICU. Crit Care Med 2015; 43:40-47

279. Guenther U, Theuerkauf N, Frommann I, et al: Predisposing and precipitating factors of delirium after cardiac surgery: A prospective observational cohort study. Ann Surg 2013; 257:1160-1167

280. van den Boogaard M, Pickkers P, Slooter AJ, et al: Development and validation of PRE-DELIRIC (PREdiction of DELIRium in ICu patients) delirium prediction model for intensive care patients: Observational multicentre study. BMJ 2012; 344:e420

281. van den Boogaard M, Schoonhoven L, Maseda E, et al: Recalibration of the delirium prediction model for ICU patients (PRE-DELIRIC): A multinational observational study. Intensive Care Med 2014; 40:361-369

282. Wassenaar A, van den Boogaard $M$, van Achterberg $T$, et al: Multinational development and validation of an early prediction model for delirium in ICU patients. Intensive Care Med 2015; 41:1048-1056

283. Balas MC, Vasilevskis EE, Olsen KM, et al: Effectiveness and safety of the awakening and breathing coordination, delirium monitoring/ management, and early exercise/mobility bundle. Crit Care Med 2014; 42:1024-1036

284. Andrews L, Silva SG, Kaplan S, et al: Delirium monitoring and patient outcomes in a general intensive care unit. Am J Crit Care 2015; 24:48-56

285. Bigatello LM, Amirfarzan H, Haghighi AK, et al: Effects of routine monitoring of delirium in a surgical/trauma intensive care unit. $J$ Trauma Acute Care Surg 2013; 74:876-883

286. van den Boogaard $M$, Pickkers $P$, van der Hoeven $H$, et al: Implementation of a delirium assessment tool in the ICU can influence haloperidol use. Crit Care 2009; 13:R131

287. Reade MC, Eastwood GM, Peck L, et al: Routine use of the Confusion Assessment Method for the Intensive Care Unit (CAMICU) by bedside nurses may underdiagnose delirium. Crit Care Resusc 2011; 13:217-224

288. Devlin JW, Fong JJ, Schumaker G, et al: Use of a validated delirium assessment tool improves the ability of physicians to identify delirium in medical intensive care unit patients. Crit Care Med 2007; 35:2721-2724; quiz 2725
289. Han JH, Eden S, Shintani A, et al: Delirium in older emergency department patients is an independent predictor of hospital length of stay. Acad Emerg Med 2011; 18:451-457

290. van Eijk MM, van Marum RJ, Klijn IA, et al: Comparison of delirium assessment tools in a mixed intensive care unit. Crit Care Med 2009; 37:1881-1885

291. Pun BT, Gordon SM, Peterson JF, et al: Large-scale implementation of sedation and delirium monitoring in the intensive care unit: $A$ report from two medical centers. Crit Care Med 2005; 33:1199-1205

292. Spronk PE, Riekerk B, Hofhuis J, et al: Occurrence of delirium is severely underestimated in the ICU during daily care. Intensive Care Med 2009; 35:1276-1280

293. Inouye SK, Foreman MD, Mion LC, et al: Nurses' recognition of delirium and its symptoms: Comparison of nurse and researcher ratings. Arch Intern Med 2001; 161:2467-2473

294. Grossmann FF, Hasemann W, Graber A, et al: Screening, detection and management of delirium in the emergency department - a pilot study on the feasibility of a new algorithm for use in older emergency department patients: The modified Confusion Assessment Method for the Emergency Department (mCAM-ED). Scand J Trauma Resusc Emerg Med 2014; 22:19

295. Svenningsen H, Egerod I, Dreyer P: Strange and scary memories of the intensive care unit: A qualitative, longitudinal study inspired by Ricoeur's interpretation theory. J Clin Nurs 2016; 25:2807-2815

296. Bélanger L, Ducharme F: Patients' and nurses' experiences of delirium: A review of qualitative studies. Nurs Crit Care 2011; 16:303-315

297. Granberg A, Engberg IB, Lundberg D: Acute confusion and unreal experiences in intensive care patients in relation to the ICU syndrome. Part II. Intensive Crit Care Nurs 1999; 15:19-33

298. Granberg A, Bergbom Engberg I, Lundberg D: Patients' experience of being critically ill or severely injured and cared for in an intensive care unit in relation to the ICU syndrome. Part I. Intensive Crit Care Nurs 1998; 14:294-307

299. Breitbart W, Gibson C, Tremblay A: The delirium experience: Delirium recall and delirium-related distress in hospitalized patients with cancer, their spouses/caregivers, and their nurses. Psychosomatics 2002; 43:183-194

300. Storli SL, Lindseth A, Asplund K: A journey in quest of meaning: A hermeneutic-phenomenological study on living with memories from intensive care. Nurs Crit Care 2008; 13:86-96

301. Gélinas C, Bérubé M, Chevrier A, et al: Delirium assessment tools in adult critically ill: A psychometric analysis and systematic review. Crit Care Nurse 2018; 38:38-49

302. Riker RR, Fugate JE; Participants in the International Multi-disciplinary Consensus Conference on Multimodality Monitoring: Clinical monitoring scales in acute brain injury: Assessment of coma, pain, agitation, and delirium. Neurocrit Care 2014; 21(Suppl 2):S27-S37

303. Nishimura K, Yokoyama K, Yamauchi N, et al; TMAD Investigators: Sensitivity and specificity of the Confusion Assessment Method for the Intensive Care Unit (CAM-ICU) and the Intensive Care Delirium Screening Checklist (ICDSC) for detecting post-cardiac surgery delirium: A single-center study in Japan. Heart Lung 2016; 45:15-20

304. Khan BA, Perkins AJ, Gao S, et al: The Confusion Assessment Method for the ICU-7 Delirium Severity Scale: A novel delirium severity instrument for use in the ICU. Crit Care Med 2017; 45:851-857

305. Tomichek JE, Stollings JL, Pandharipande PP, et al: Antipsychotic prescribing patterns during and after critical illness: A prospective cohort study. Crit Care 2016; 20:378

306. Marshall J, Herzig SJ, Howell MD, et al: Antipsychotic utilization in the intensive care unit and in transitions of care. J Crit Care 2016; 33:119-124

307. Herzig SJ, Rothberg MB, Guess JR, et al: Antipsychotic medication utilization in nonpsychiatric hospitalizations. J Hosp Med 2016; 11:543-549

308. Botha JA, Mudholkar P: The effect of a sedation scale on ventilation hours, sedative, analgesic and inotropic use in an intensive care unit. Crit Care Resusc 2004; 6:253-257

309. Gélinas C, Arbour C, Michaud C, et al: Implementation of the CriticalCare Pain Observation Tool on pain assessment/management nursing practices in an intensive care unit with nonverbal critically ill adults: A before and after study. Int J Nurs Stud 2011; 48:1495-1504 
310. Radtke FM, Heymann A, Franck M, et al: How to implement monitoring tools for sedation, pain and delirium in the intensive care unit: An experimental cohort study. Intensive Care Med 2012; 38:1974-1981

311. Luetz A, Weiss B, Boettcher $S$, et al: Routine delirium monitoring is independently associated with a reduction of hospital mortality in critically ill surgical patients: A prospective, observational cohort study. J Crit Care 2016; 35:168-173

312. Patel SB, Poston JT, Pohlman A, et al: Rapidly reversible, sedationrelated delirium versus persistent delirium in the intensive care unit. Am J Respir Crit Care Med 2014; 189:658-665

313. Haenggi M, Blum S, Brechbuehl R, et al: Effect of sedation level on the prevalence of delirium when assessed with CAM-ICU and ICDSC. Intensive Care Med 2013; 39:2171-2179

314. Gusmao-Flores D, Martins JC, Amorin D, et al: Tools for diagnosing delirium in the critically ill: Is calibration needed for the less sedated patient? Intensive Care Med 2014; 40:137-138

315. Svenningsen $\mathrm{H}$, Egerod I, Videbech P, et al: Fluctuations in sedation levels may contribute to delirium in ICU patients. Acta Anaesthesiol Scand 2013; 57:288-293

316. Ely EW, Shintani A, Truman B, et al: Delirium as a predictor of mortality in mechanically ventilated patients in the intensive care unit. JAMA 2004; 291:1753-1762

317. Pandharipande PP, Girard TD, Jackson JC, et al; BRAIN-ICU Study Investigators: Long-term cognitive impairment after critical illness. $N$ Engl J Med 2013; 369:1306-1316

318. Wolters AE, van Dijk D, Pasma W, et al: Long-term outcome of delirium during intensive care unit stay in survivors of critical illness: A prospective cohort study. Crit Care 2014; 18:R125

319. Girard TD, Jackson JC, Pandharipande PP, et al: Delirium as a predictor of long-term cognitive impairment in survivors of critical illness. Crit Care Med 2010; 38:1513-1520

320. Ely EW, Gautam S, Margolin R, et al: The impact of delirium in the intensive care unit on hospital length of stay. Intensive Care Med $2001 ; 27: 1892-1900$

321. Lat I, McMillian W, Taylor S, et al: The impact of delirium on clinical outcomes in mechanically ventilated surgical and trauma patients. Crit Care Med 2009; 37:1898-1905

322. Naidech AM, Beaumont JL, Rosenberg NF, et al: Intracerebral hemorrhage and delirium symptoms. Length of stay, function, and quality of life in a 114-patient cohort. Am J Respir Crit Care Med 2013; 188:1331-1337

323. Thomason JW, Shintani A, Peterson JF, et al: Intensive care unit delirium is an independent predictor of longer hospital stay: A prospective analysis of 261 non-ventilated patients. Crit Care 2005; 9:R375-R381

324. Alexander SA, Ren D, Gunn SR, et al: Interleukin 6 and apolipoprotein $E$ as predictors of acute brain dysfunction and survival in critical care patients. Am J Crit Care 2014; 23:49-57

325. Milbrandt EB, Deppen S, Harrison PL, et al: Costs associated with delirium in mechanically ventilated patients. Crit Care Med 2004; 32:955-962

326. Mu DL, Wang DX, Li LH, et al: High serum cortisol level is associated with increased risk of delirium after coronary artery bypass graft surgery: A prospective cohort study. Crit Care 2010; 14:R238

327. Ouimet S, Kavanagh BP, Gottfried SB, et al: Incidence, risk factors and consequences of ICU delirium. Intensive Care Med 2007; 33:66-73

328. Bienvenu OJ, Gellar J, Althouse BM, et al: Post-traumatic stress disorder symptoms after acute lung injury: A 2-year prospective longitudinal study. Psychol Med 2013; 43:2657-2671

329. Wolters AE, Peelen LM, Welling MC, et al: Long-term mental health problems after delirium in the ICU. Crit Care Med 2016; $44: 1808-1813$

330. Jackson JC, Pandharipande PP, Girard TD, et al; Bringing to Light the Risk Factors and Incidence of Neuropsychological Dysfunction in ICU Survivors (BRAIN-ICU) Study Investigators: Depression, post-traumatic stress disorder, and functional disability in survivors of critical illness in the BRAIN-ICU study: A longitudinal cohort study. Lancet Respir Med 2014; 2:369-379
331. Girard TD, Shintani AK, Jackson JC, et al: Risk factors for post-traumatic stress disorder symptoms following critical illness requiring mechanical ventilation: A prospective cohort study. Crit Care 2007; $11: R 28$

332. Patel MB, Jackson JC, Morandi A, et al: Incidence and risk factors for intensive care unit-related post-traumatic stress disorder in Veterans and civilians. Am J Respir Crit Care Med 2016; 193:1373-1381

333. Roberts BL, Rickard CM, Rajbhandari D, et al: Factual memories of ICU: Recall at two years post-discharge and comparison with delirium status during ICU admission-a multicentre cohort study. J Clin Nurs 2007; 16:1669-1677

334. van den Boogaard M, Schoonhoven L, Evers AW, et al: Delirium in critically ill patients: Impact on long-term health-related quality of life and cognitive functioning. Crit Care Med 2012; 40:112-118

335. Roberts BL, Rickard CM, Rajbhandari D, et al: Patients' dreams in ICU: Recall at two years post discharge and comparison to delirium status during ICU admission. A multicentre cohort study. Intensive Crit Care Nurs 2006; 22:264-273

336. Van Rompaey B, Schuurmans MJ, Shortridge-Baggett LM, et al: Long term outcome after delirium in the intensive care unit. $J$ Clin Nurs 2009; 18:3349-3357

337. Shehabi Y, Riker RR, Bokesch PM, et al; SEDCOM (Safety and Efficacy of Dexmedetomidine Compared With Midazolam) Study Group: Delirium duration and mortality in lightly sedated, mechanically ventilated intensive care patients. Crit Care Med 2010; 38:2311-2318

338. Tsuruta R, Oda Y, Shintani A, et al; Japanese Epidemiology of Delirium in ICUs (JEDI) Study Investigators: Delirium and coma evaluated in mechanically ventilated patients in the intensive care unit in Japan: A multi-institutional prospective observational study. J Crit Care 2014; 29:472.e1-472.e5

339. Caruso P, Guardian L, Tiengo T, et al: ICU architectural design affects the delirium prevalence: A comparison between single-bed and multibed rooms. Crit Care Med 2014; 42:2204-2210

340. Guillamondegui OD, Richards JE, Ely EW, et al: Does hypoxia affect intensive care unit delirium or long-term cognitive impairment after multiple trauma without intracranial hemorrhage? J Trauma 2011; 70:910-915

341. Mardani D, Bigdelian H: Predictors and clinical outcomes of postoperative delirium after administration of dexamethasone in patients undergoing coronary artery bypass surgery. Int J Prev Med 2012; 3:420-427

342. Ouimet S, Riker R, Bergeron N, et al: Subsyndromal delirium in the ICU: Evidence for a disease spectrum. Intensive Care Med 2007; 33:1007-1013

343. Roberts B, Rickard CM, Rajbhandari D, et al: Multicentre study of delirium in ICU patients using a simple screening tool. Aust Crit Care 2005; 18:6, 8-9, 11

344. Robinson TN, Raeburn CD, Tran ZV, et al: Motor subtypes of postoperative delirium in older adults. Arch Surg 2011; 146:295-300

345. Salluh JI, Soares M, Teles JM, et al; Delirium Epidemiology in Critical Care Study Group: Delirium Epidemiology in Critical Care (DECCA): An international study. Crit Care 2010; 14:R210

346. Serafim RB, Dutra MF, Saddy F, et al: Delirium in postoperative nonventilated intensive care patients: Risk factors and outcomes. Ann Intensive Care 2012; 2:51

347. Sharma A, Malhotra S, Grover S, et al: Incidence, prevalence, risk factor and outcome of delirium in intensive care unit: A study from India. Gen Hosp Psychiatry 2012; 34:639-646

348. Simons KS, Workum JD, Slooter AJ, et al: Effect of preadmission sunlight exposure on intensive care unit-acquired delirium: A multicenter study. J Crit Care 2014; 29:283-286

349. van den Boogaard M, Peters SA, van der Hoeven JG, et al: The impact of delirium on the prediction of in-hospital mortality in intensive care patients. Crit Care 2010; 14:R146

350. Yamaguchi T, Tsukioka E, Kishi Y: Outcomes after delirium in a Japanese intensive care unit. Gen Hosp Psychiatry 2014; 36:634-636

351. Dubois MJ, Bergeron N, Dumont $M$, et al: Delirium in an intensive care unit: A study of risk factors. Intensive Care Med 2001; 27:1297-1304

352. Lin SM, Huang CD, Liu CY, et al: Risk factors for the development of early-onset delirium and the subsequent clinical outcome in mechanically ventilated patients. J Crit Care 2008; 23:372-379 
353. Balas MC, Happ MB, Yang W, et al: Outcomes associated with delirium in older patients in surgical ICUs. Chest 2009; 135:18-25

354. Balas MC, Chaperon C, Sisson JH, et al: Transitions experienced by older survivors of critical care. J Gerontol Nurs 2011; 37:14-25; quiz 26

355. Marquis F, Ouimet S, Riker R, et al: Individual delirium symptoms: Do they matter? Crit Care Med 2007; 35:2533-2537

356. Abraham CM, Obremskey WT, Song Y, et al: Hospital delirium and psychological distress at 1 year and health-related quality of life after moderate-to-severe traumatic injury without intracranial hemorrhage. Arch Phys Med Rehabil 2014; 95:2382-2389

357. Abelha FJ, Luis C, Veiga D, et al: Outcome and quality of life in patients with postoperative delirium during an ICU stay following major surgery. Crit Care 2013; 17:R257

358. Brummel NE, Jackson JC, Pandharipande PP, et al: Delirium in the ICU and subsequent long-term disability among survivors of mechanical ventilation. Crit Care Med 2014; 42:369-377

359. Norman BC, Jackson JC, Graves JA, et al: Employment outcomes after critical illness: An analysis of the bringing to light the risk factors and incidence of neuropsychological dysfunction in ICU survivors cohort. Crit Care Med 2016; 44:2003-2009

360. Svenningsen $H$, Tønnesen EK, Videbech $P$, et al: Intensive care delirium - effect on memories and health-related quality of life - a followup study. J Clin Nurs 2014; 23:634-644

361. Tomasi CD, Grandi C, Salluh J, et al: Comparison of CAM-ICU and ICDSC for the detection of delirium in critically ill patients focusing on relevant clinical outcomes. J Crit Care 2012; 27:212-217

362. Veiga $D$, Luis $C$, Parente $D$, et al: Postoperative delirium in intensive care patients: Risk factors and outcome. Rev Bras Anestesio/ 2012; 62:469-483

363. Lin SM, Liu CY, Wang CH, et al: The impact of delirium on the survival of mechanically ventilated patients. Crit Care Med 2004; 32:2254-2259

364. Pisani MA, Kong SY, KasI SV, et al: Days of delirium are associated with 1 -year mortality in an older intensive care unit population. $\mathrm{Am} \mathrm{J}$ Respir Crit Care Med 2009; 180:1092-1097

365. Klein Klouwenberg PM, Zaal IJ, Spitoni C, et al: The attributable mortality of delirium in critically ill patients: Prospective cohort study. BMJ 2014; 349:g6652

366. Wang W, Li HL, Wang DX, et al: Haloperidol prophylaxis decreases delirium incidence in elderly patients after noncardiac surgery: A randomized controlled trial. Crit Care Med 2012; 40:731-739

367. Prakanrattana U, Prapaitrakool S: Efficacy of risperidone for prevention of postoperative delirium in cardiac surgery. Anaesth Intensive Care 2007; 35:714-719

368. Su X, Meng ZT, Wu XH, et al: Dexmedetomidine for prevention of delirium in elderly patients after non-cardiac surgery: $A$ randomised, double-blind, placebo-controlled trial. Lancet 2016; 388:1893-1902

369. van den Boorgard M, Slooter AJC, Bruggemann RJM, et al: Effect of prophylactic haloperidol on survival among critically ill adults at high risk for delirium: The REDUCE randomized clinical trial. JAMA 2018; 319:680-690

370. Skrobik Y, Duprey MS, Hill NS, et al: Low-dose nocturnal dexmedetomidine prevents ICU delirium. A randomized, placebo-controlled trial. Am J Respir Crit Care Med 2018; 197:1147-1156

371. Page VJ, Davis D, Zhao XB, et al: Statin use and risk of delirium in the critically ill. Am J Respir Crit Care Med 2014; 189:666-673

372. Morandi A, Hughes CG, Girard TD, et al: Statins and brain dysfunction: A hypothesis to reduce the burden of cognitive impairment in patients who are critically ill. Chest $2011 ; 140: 580-585$

373. Mather JF, Corradi JP, Waszynski C, et al: Statin and its association with delirium in the medical ICU. Crit Care Med 2017; 45:1515-1522

374. Billings FT 4th, Hendricks PA, Schildcrout JS, et al: High-dose perioperative atorvastatin and acute kidney injury following cardiac surgery: A randomized clinical trial. JAMA 2016; 315:877-888

375. Avidan MS, Maybrier HR, Abdallah AB, et al; PODCAST Research Group: Intraoperative ketamine for prevention of postoperative delirium or pain after major surgery in older adults: An international, multicentre, double-blind, randomised clinical trial. Lancet 2017; 390:267-275

376. Brummel NE, Boehm LM, Girard TD, et al: Subsyndromal delirium and institutionalization among patients with critical illness. Am J Crit Care 2017; 26:447-455

377. Al-Qadheeb NS, Skrobik Y, Schumaker G, et al: Preventing ICU subsyndromal delirium conversion to delirium with low-dose IV haloperidol: A double-blind, placebo-controlled pilot study. Crit Care Med 2016; 44:583-591

378. Hakim SM, Othman Al, Naoum DO: Early treatment with risperidone for subsyndromal delirium after on-pump cardiac surgery in the elderly: A randomized trial. Anesthesiology 2012; 116:987-997

379. Devlin JW, Smithburger P, Kane JM, et al: Intended and unintended consequences of constraining clinician prescribing: The case of antipsychotics. Crit Care Med 2016; 44:1805-1807

380. Girard TD, Pandharipande PP, Carson SS, et al; MIND Trial Investigators: Feasibility, efficacy, and safety of antipsychotics for intensive care unit delirium: The MIND randomized, placebo-controlled trial. Crit Care Med 2010; 38:428-437

381. Page VJ, Ely EW, Gates S, et al: Effect of intravenous haloperidol on the duration of delirium and coma in critically ill patients (Hope-ICU): A randomised, double-blind, placebo-controlled trial. Lancet Respir Med 2013; 1:515-523

382. Devlin JW, Roberts RJ, Fong JJ, et al: Efficacy and safety of quetiapine in critically ill patients with delirium: A prospective, multicenter, randomized, double-blind, placebo-controlled pilot study. Crit Care Med 2010; 38:419-427

383. Skrobik YK, Bergeron N, Dumont M, et al: Olanzapine vs haloperidol: Treating delirium in a critical care setting. Intensive Care Med 2004; 30:444-449

384. Needham DM, Colantuoni E, Dinglas VD, et al: Rosuvastatin versus placebo for delirium in intensive care and subsequent cognitive impairment in patients with sepsis-associated acute respiratory distress syndrome: An ancillary study to a randomised controlled trial. Lancet Respir Med 2016; 4:203-212

385. Page VJ, Casarin A, Ely EW, et al: Evaluation of early administration of simvastatin in the prevention and treatment of delirium in critically ill patients undergoing mechanical ventilation (MoDUS): A randomised, doubleblind, placebo-controlled trial. Lancet Respir Med 2017; 1:515-523

386. Reade MC, Eastwood GM, Bellomo R, et al; DahLIA Investigators; Australian and New Zealand Intensive Care Society Clinical Trials Group: Effect of dexmedetomidine added to standard care on ventilator-free time in patients with agitated delirium: A randomized clinical trial. JAMA 2016; 315:1460-1468

387. Ono H, Taguchi T, Kido Y, et al: The usefulness of bright light therapy for patients after oesophagectomy. Intensive Crit Care Nurs 2011; 27:158-166

388. Taguchi T, Yano M, Kido Y: Influence of bright light therapy on postoperative patients: A pilot study. Intensive Crit Care Nurs 2007; 23:289-297

389. Simons KS, Laheij RJ, van den Boogaard M, et al: Dynamic light application therapy to reduce the incidence and duration of delirium in intensive-care patients: A randomised controlled trial. Lancet Respir Med 2016; 4:194-202

390. Black P, Boore JR, Parahoo K: The effect of nurse-facilitated family participation in the psychological care of the critically ill patient. J Adv Nurs 2011; 67:1091-1101

391. Brummel NE, Girard TD, Ely EW, et al: Feasibility and safety of early combined cognitive and physical therapy for critically ill medical and surgical patients: The Activity and Cognitive Therapy in ICU (ACTICU) trial. Intensive Care Med 2014; 40:370-379

392. Foster J, Kelly M: A pilot study to test the feasibility of a nonpharmacologic intervention for the prevention of delirium in the medical intensive care unit. Clin Nurse Spec 2013; 27:231-238

393. Moon KJ, Lee SM: The effects of a tailored intensive care unit delirium prevention protocol: A randomized controlled trial. Int J Nurs Stud 2015; 52:1423-1432

394. Colombo R, Corona A, Praga F, et al: A reorientation strategy for reducing delirium in the critically ill. Results of an interventional study. Minerva Anestesio/ 2012; 78:1026-1033 
395. Hanison J, Conway D: A multifaceted approach to prevention of delirium on intensive care. BMJ Qual Improv Rep 2015; 12:4-12

396. Rivosecchi RM, Kane-Gill SL, Svec S, et al: The implementation of a nonpharmacologic protocol to prevent intensive care delirium. J Crit Care 2016; 31:206-211

397. Denehy L, Lanphere J, Needham DM: Ten reasons why ICU patients should be mobilized early. Intensive Care Med 2017; 43:86-90

398. Fan E, Dowdy DW, Colantuoni E, et al: Physical complications in acute lung injury survivors: A two-year longitudinal prospective study. Crit Care Med 2014; 42:849-859

399. Hermans G, Van Mechelen H, Clerckx B, et al: Acute outcomes and 1-year mortality of intensive care unit-acquired weakness. A cohort study and propensity-matched analysis. Am J Respir Crit Care Med 2014; 190:410-420

400. Dinglas VD, Aronson Friedman L, Colantuoni E, et al: Muscle weakness and 5-year survival in acute respiratory distress syndrome survivors. Crit Care Med 2017; 45:446-453

401. Needham DM, Wozniak AW, Hough CL, et al; National Institutes of Health NHLBI ARDS Network: Risk factors for physical impairment after acute lung injury in a national, multicenter study. Am J Respir Crit Care Med 2014; 189:1214-1224

402. Kamdar BB, Combs MP, Colantuoni E, et al: The association of sleep quality, delirium, and sedation status with daily participation in physical therapy in the ICU. Crit Care 2016; 19:261

403. Latronico N, Herridge M, Hopkins RO, et al: The ICM research agenda on intensive care unit-acquired weakness. Intensive Care Med 2017; 43:1270-1281

404. World Health Organization: Rehabilitation: Key for health in the 21 st century. Geneva, Switzerland, World Health Organization, 2017

405. Amidei C: Mobilisation in critical care: A concept analysis. Intensive Crit Care Nurs 2012; 28:73-81

406. Patman S, Sanderson D, Blackmore M: Physiotherapy following cardiac surgery: Is it necessary during the intubation period? Aust $J$ Physiother 2001; 47:7-16

407. Morris PE, Goad A, Thompson C, et al: Early intensive care unit mobility therapy in the treatment of acute respiratory failure. Crit Care Med 2008; 36:2238-2243

408. Schweickert WD, Pohlman MC, Pohlman AS, et al: Early physical and occupational therapy in mechanically ventilated, critically ill patients: A randomised controlled trial. Lancet 2009; 373:1874-1882

409. Routsi C, Gerovasili V, Vasileiadis I, et al: Electrical muscle stimulation prevents critical illness polyneuromyopathy: A randomized parallel intervention trial. Crit Care 2010; 14:R74

410. Dantas CM, Silva PF, Siqueira FH, et al: Influence of early mobilization on respiratory and peripheral muscle strength in critically ill patients. Rev Bras Ter Intensiva 2012; 24:173-178

411. Denehy L, Skinner EH, Edbrooke L, et al: Exercise rehabilitation for patients with critical illness: A randomized controlled trial with 12 months of follow-up. Crit Care 2013; 17:R156

412. Ali MS, Talwar D, Jain SK: The effect of a short-term pulmonary rehabilitation on exercise capacity and quality of life in patients hospitalised with acute exacerbation of chronic obstructive pulmonary disease. Indian J Chest Dis Allied Sci 2014; 56:13-19

413. Dong ZH, Yu BX, Sun YB, et al: Effects of early rehabilitation therapy on patients with mechanical ventilation. World J Emerg Med 2014; 5:48-52

414. Kayambu G, Boots R, Paratz J: Early physical rehabilitation in intensive care patients with sepsis syndromes: A pilot randomised controlled trial. Intensive Care Med 2015; 41:865-874

415. Kho ME, Truong AD, Zanni JM, et al: Neuromuscular electrical stimulation in mechanically ventilated patients: A randomized, shamcontrolled pilot trial with blinded outcome assessment. J Crit Care 2015; 30:32-39

416. Moss $M$, Nordon-Craft A, Malone D, et al: A randomized trial of an intensive physical therapy program for patients with acute respiratory failure. Am J Respir Crit Care Med 2016; 193:1101-1110

417. Morris PE, Berry MJ, Files DC, et al: Standardized rehabilitation and hospital length of stay among patients with acute respiratory failure: A randomized clinical trial. JAMA 2016; 315:2694-2702
418. Burtin C, Clerckx B, Robbeets C, et al: Early exercise in critically ill patients enhances short-term functional recovery. Crit Care Med 2009; 37:2499-2505

419. Yosef-Brauner O, Adi N, Ben Shahar T, et al: Effect of physical therapy on muscle strength, respiratory muscles and functional parameters in patients with intensive care unit-acquired weakness. Clin Respir J 2015; 9:1-6

420. Hodgson CL, Bailey M, Bellomo R, et al; Trial of Early Activity and Mobilization Study Investigators: A binational multicenter pilot feasibility randomized controlled trial of early goal-directed mobilization in the ICU. Crit Care Med 2016; 44:1145-1152

421. Burns KE, Jacob SK, Aguirre V, et al: Stakeholder engagement in trial design: Survey of visitors to critically ill patients regarding preferences for outcomes and treatment options during weaning from mechanical ventilation. Ann Am Thorac Soc 2016; 13:1962-1968

422. Stiller K, Phillips A, Lambert $P$ : The safety of mobilisation and its effect on haemodynamic and respiratory status of intensive care patients. Physiother Theory Pract 2004; 20:175-185

423. Zanni JM, Korupolu R, Fan E, et al: Rehabilitation therapy and outcomes in acute respiratory failure: An observational pilot project. $J$ Crit Care 2010; 25:254-262

424. Kho ME, Damluji A, Zanni JM, et al: Feasibility and observed safety of interactive video games for physical rehabilitation in the intensive care unit: A case series. J Crit Care 2012; 27:219.e1-219.e6

425. Berney S, Haines K, Skinner EH, et al: Safety and feasibility of an exercise prescription approach to rehabilitation across the continuum of care for survivors of critical illness. Phys Ther 2012; 92:1524-1535

426. Damluji A, Zanni JM, Mantheiy E, et al: Safety and feasibility of femoral catheters during physical rehabilitation in the intensive care unit. J Crit Care 2013; 28:535.e9-535.15

427. Sricharoenchai T, Parker AM, Zanni JM, et al: Safety of physical therapy interventions in critically ill patients: A single-center prospective evaluation of 1110 intensive care unit admissions. J Crit Care 2014; 29:395-400

428. Berney S, Skinner EH, Denehy L, et al: Development of a physical function outcome measure (PFIT) and a pilot exercise training protocol for use in intensive care. Crit Care Resusc 2009; 11:110

429. Pohlman MC, Schweickert WD, Pohlman AS, et al: Feasibility of physical and occupational therapy beginning from initiation of mechanical ventilation. Crit Care Med 2010; 38:2089-2094

430. Nydahl P, Sricharoenchai T, Chandra S, et al: Safety of patient mobilization and rehabilitation in the intensive care unit. Systematic review with meta-analysis. Ann Am Thorac Soc 2017; 14:766-777

431. Fowlow B, Price P, Fung T: Ambulation after sheath removal: A comparison of 6 and 8 hours of bedrest after sheath removal in patients following a PTCA procedure. Heart Lung 1995; 24:28-37

432. Hildreth AN, Enniss T, Martin RS, et al: Surgical intensive care unit mobility is increased after institution of a computerized mobility order set and intensive care unit mobility protocol: A prospective cohort analysis. Am Surg 2010; 76:818-822

433. Hanekom S, Louw QA, Coetzee AR: Implementation of a protocol facilitates evidence-based physiotherapy practice in intensive care units. Physiotherapy 2013; 99:139-145

434. Pires-Neto RC, Pereira AL, Parente C, et al: Characterization of the use of a cycle ergometer to assist in the physical therapy treatment of critically ill patients. Rev Bras Ter Intensiva 2013; 25:39-43

435. Dickinson S, Tschannen D, Shever LL: Can the use of an early mobility program reduce the incidence of pressure ulcers in a surgical critical care unit? Crit Care Nurs Q 2013; 36:127-140

436. Simini B: Patients' perceptions of intensive care. Lancet 1999; 354:571-572

437. Rotondi AJ, Chelluri L, Sirio C, et al: Patients' recollections of stressful experiences while receiving prolonged mechanical ventilation in an intensive care unit. Crit Care Med 2002; 30:746-752

438. Cooper AB, Thornley KS, Young GB, et al: Sleep in critically ill patients requiring mechanical ventilation. Chest 2000; 117:809-818

439. Aurell J, Elmqvist D: Sleep in the surgical intensive care unit: Continuous polygraphic recording of sleep in nine patients receiving postoperative care. Br Med J (Clin Res Ed) 1985; 290:1029-1032 
440. Freedman NS, Gazendam J, Levan L, et al: Abnormal sleep/wake cycles and the effect of environmental noise on sleep disruption in the intensive care unit. Am J Respir Crit Care Med 2001; 163:451-457

441. Helton MC, Gordon SH, Nunnery SL: The correlation between sleep deprivation and the intensive care unit syndrome. Heart Lung 1980; 9:464-468

442. Trompeo AC, Vidi Y, Locane MD, et al: Sleep disturbances in the critically ill patients: Role of delirium and sedative agents. Minerva Anestesio/ 2011; 77:604-612

443. Roche Campo F, Drouot X, Thille AW, et al: Poor sleep quality is associated with late noninvasive ventilation failure in patients with acute hypercapnic respiratory failure. Crit Care Med 2010; 38:477-485

444. White DP, Douglas NJ, Pickett CK, et al: Sleep deprivation and the control of ventilation. Am Rev Respir Dis 1983; 128:984-986

445. Benca RM, Quintas J: Sleep and host defenses: A review. Sleep 1997; 20:1027-1037

446. Bryant PA, Trinder J, Curtis N: Sick and tired: Does sleep have a vital role in the immune system? Nat Rev Immunol 2004; 4:457-467

447. Boesen $\mathrm{HC}$, Andersen $\mathrm{JH}$, Bendtsen $\mathrm{AO}$, et al: Sleep and delirium in unsedated patients in the intensive care unit. Acta Anaesthesiol Scand 2016; 60:59-68

448. Beecroft JM, Ward M, Younes M, et al: Sleep monitoring in the intensive care unit: Comparison of nurse assessment, actigraphy and polysomnography. Intensive Care Med 2008; 34:2076-2083

449. Elliott R, McKinley S, Cistulli $P$, et al: Characterisation of sleep in intensive care using 24-hour polysomnography: An observational study. Crit Care 2013; 17:R46

450. Knauert MP, Yaggi HK, Redeker NS, et al: Feasibility study of unattended polysomnography in medical intensive care unit patients. Heart Lung 2014; 43:445-452

451. Roche-Campo F, Thille AW, Drouot X, et al: Comparison of sleep quality with mechanical versus spontaneous ventilation during weaning of critically III tracheostomized patients. Crit Care Med 2013; 41:1637-1644

452. Drouot X, Bridoux A, Thille AW, et al: Sleep continuity: A new metric to quantify disrupted hypnograms in non-sedated intensive care unit patients. Crit Care 2014; 18:628

453. Gabor JY, Cooper AB, Crombach SA, et al: Contribution of the intensive care unit environment to sleep disruption in mechanically ventilated patients and healthy subjects. Am J Respir Crit Care Med 2003; 167:708-715

454. Córdoba-Izquierdo A, Drouot X, Thille AW, et al: Sleep in hypercapnic critical care patients under noninvasive ventilation: Conventional versus dedicated ventilators. Crit Care Med 2013; 41:60-68

455. Freedman NS, Kotzer N, Schwab RJ: Patient perception of sleep quality and etiology of sleep disruption in the intensive care unit. $A m$ J Respir Crit Care Med 1999; 159:1155-1162

456. Little A, Ethier C, Ayas N, et al: A patient survey of sleep quality in the intensive care unit. Minerva Anestesiol 2012; 78:406-414

457. Drouot X, Roche-Campo F, Thille AW, et al: A new classification for sleep analysis in critically ill patients. Sleep Med 2012; 13:7-14

458. Van Rompaey B, Elseviers MM, Van Drom W, et al: The effect of earplugs during the night on the onset of delirium and sleep perception: $A$ randomized controlled trial in intensive care patients. Crit Care 2012; 16:R73

459. Kamdar BB, King LM, Collop NA, et al: The effect of a quality improvement intervention on perceived sleep quality and cognition in a medical ICU. Crit Care Med 2013; 41:800-809

460. Elliott R, Rai T, McKinley S: Factors affecting sleep in the critically ill: An observational study. J Crit Care 2014; 29:859-863

461. Friese RS, Diaz-Arrastia R, McBride D, et al: Quantity and quality of sleep in the surgical intensive care unit: Are our patients sleeping? J Trauma 2007; 63:1210-1214

462. Bosma K, Ferreyra G, Ambrogio C, et al: Patient-ventilator interaction and sleep in mechanically ventilated patients: Pressure support versus proportional assist ventilation. Crit Care Med 2007; 35:1048-1054

463. Knauert MP, Malik V, Kamdar BB: Sleep and sleep disordered breathing in hospitalized patients. Semin Respir Crit Care Med $2014 ; 35: 582-592$
464. Kondili E, Alexopoulou C, Xirouchaki N, et al: Effects of propofol on sleep quality in mechanically ventilated critically ill patients: A physiological study. Intensive Care Med 2012; 38:1640-1646

465. Delisle $S$, Ouellet $P$, Bellemare $P$, et al: Sleep quality in mechanically ventilated patients: Comparison between NAVA and PSV modes. Ann Intensive Care 2011; 1:42

466. Hardin KA, Seyal M, Stewart T, et al: Sleep in critically ill chemically paralyzed patients requiring mechanical ventilation. Chest 2006; 129:1468-1477

467. Oto J, Yamamoto K, Koike S, et al: Effect of daily sedative interruption on sleep stages of mechanically ventilated patients receiving midazolam by infusion. Anaesth Intensive Care 2011; 39:392-400

468. Alexopoulou C, Kondili E, Vakouti E, et al: Sleep during proportionalassist ventilation with load-adjustable gain factors in critically ill patients. Intensive Care Med 2007; 33:1139-1147

469. Toublanc B, Rose D, Glérant JC, et al: Assist-control ventilation vs. low levels of pressure support ventilation on sleep quality in intubated ICU patients. Intensive Care Med 2007; 33:1148-1154

470. Alexopoulou C, Kondili E, Diamantaki E, et al: Effects of dexmedetomidine on sleep quality in critically ill patients: A pilot study. Anesthesiology 2014; 121:801-807

471. Alexopoulou C, Kondili E, Plataki M, et al: Patient-ventilator synchrony and sleep quality with proportional assist and pressure support ventilation. Intensive Care Med 2013; 39:1040-1047

472. Andréjak C, Monconduit J, Rose D, et al: Does using pressure-controlled ventilation to rest respiratory muscles improve sleep in ICU patients? Respir Med 2013; 107:534-541

473. Cabello B, Thille AW, Drouot X, et al: Sleep quality in mechanically ventilated patients: Comparison of three ventilatory modes. Crit Care Med 2008; 36:1749-1755

474. Parthasarathy S, Friese RS, Ayas NT: Biological validity to sleep measurements during critical illness. Crit Care Med 2010; 38:705-706

475. Rechtschaffen A, Kales A: A Manual of Standardized Terminology, Techniques and Scoring System for Sleep Stages of Human Subjects. Washington, DC, Public Health Service, 1968. Cited August 30, 2017. Available at: http://ci.nii.ac.jp/naid/10023901533/. Accessed June 14, 2017

476. Watson PL, Pandharipande P, Gehlbach BK, et al: Atypical sleep in ventilated patients: Empirical electroencephalography findings and the path toward revised ICU sleep scoring criteria. Crit Care Med 2013; 41:1958-1967

477. Cochen V, Arnulf I, Demeret S, et al: Vivid dreams, hallucinations, psychosis and REM sleep in Guillain-Barré syndrome. Brain 2005; 128:2535-2545

478. Freedman RR, Kruger ML, Wasson SL: Heart rate variability in menopausal hot flashes during sleep. Menopause 2011; 18:897-900

479. Sutter R, Barnes B, Leyva A, et al: Electroencephalographic sleep elements and outcome in acute encephalopathic patients: A 4-year cohort study. Eur J Neuro/ 2014; 21:1268-1275

480. Valente M, Placidi F, Oliveira AJ, et al: Sleep organization pattern as a prognostic marker at the subacute stage of post-traumatic coma. Clin Neurophysio/ 2002; 113:1798-1805

481. Foreman B, Westwood AJ, Claassen J, et al: Sleep in the neurological intensive care unit: Feasibility of quantifying sleep after melatonin supplementation with environmental light and noise reduction. $J$ Clin Neurophysio/ 2015; 32:66-74

482. Bihari S, Doug McEvoy R, Matheson E, et al: Factors affecting sleep quality of patients in intensive care unit. J Clin Sleep Med 2012; 8:301-307

483. McKinley S, Fien M, Elliott R, et al: Sleep and psychological health during early recovery from critical illness: An observational study. $J$ Psychosom Res 2013; 75:539-545

484. Armutcu B, Celik T: Sleep quality of patients hospitalized in the coronary intensive care unit and the affecting factors. Int J Caring Sci 2014; 7:324

485. Simpson T, Lee ER, Cameron C: Patients' perceptions of environmental factors that disturb sleep after cardiac surgery. Am J Crit Care 1996; 5:173-181

486. Nicolás A, Aizpitarte E, Iruarrizaga A, et al: Perception of night-time sleep by surgical patients in an intensive care unit. Nurs Crit Care 2008; 13:25-33 
487. Hofhuis JG, Spronk PE, van Stel HF, et al: Experiences of critically ill patients in the ICU. Intensive Crit Care Nurs 2008; 24:300-313

488. Ehlers VJ, Watson H, Moleki MM: Factors contributing to sleep deprivation in a multidisciplinary intensive care unit in South Africa. Curationis 2013; 36:E1-E8

489. Yinnon AM, Ilan $Y$, Tadmor B, et al: Quality of sleep in the medical department. Br J Clin Pract 1992; 46:88-91

490. Uğraş GA, Oztekin SD: Patient perception of environmental and nursing factors contributing to sleep disturbances in a neurosurgical intensive care unit. Tohoku J Exp Med 2007; 212:299-308

491. Richards KC, Anderson WM, Chesson AL Jr, et al: Sleep-related breathing disorders in patients who are critically ill. J Cardiovasc Nurs 2002; 17:42-55

492. Frisk U, Nordström G: Patients' sleep in an intensive care unitpatients' and nurses' perception. Intensive Crit Care Nurs 2003; 19:342-349

493. Zhang L, Sha YS, Kong QQ, et al: Factors that affect sleep quality: Perceptions made by patients in the intensive care unit after thoracic surgery. Support Care Cancer 2013; 21:2091-2096

494. Fanfulla F, Ceriana P, D'Artavilla Lupo N, et al: Sleep disturbances in patients admitted to a step-down unit after ICU discharge: The role of mechanical ventilation. Sleep 2011; 34:355-362

495. Parthasarathy S, Tobin MJ: Effect of ventilator mode on sleep quality in critically ill patients. Am J Respir Crit Care Med 2002; 166:1423-1429

496. Zhang WY, Wu WL, Gu JJ, et al: Risk factors for postoperative delirium in patients after coronary artery bypass grafting: A prospective cohort study. J Crit Care 2015; 30:606-612

497. Bryczkowski SB, Lopreiato MC, Yonclas PP, et al: Delirium prevention program in the surgical intensive care unit improved the outcomes of older adults. J Surg Res 2014; 190:280-288

498. Duclos $C$, Dumont M, Blais $H$, et al: Rest-activity cycle disturbances in the acute phase of moderate to severe traumatic brain injury. Neurorehabil Neural Repair 2014; 28:472-482

499. Van den Broecke S, Jobard O, Montalescot G, et al: Very early screening for sleep-disordered breathing in acute coronary syndrome in patients without acute heart failure. Sleep Med 2014; 15:1539-1546

500. Saito T, Yoshikawa T, Sakamoto Y, et al: Sleep apnea in patients with acute myocardial infarction. Crit Care Med 1991; 19:938-941

501. Mistraletti G, Taverna M, Sabbatini G, et al: Actigraphic monitoring in critically ill patients: Preliminary results toward an "observationguided sedation." J Crit Care 2009; 24:563-567

502. BaHammam A, Syed S, Al-Mughairy A: Sleep-related breathing disorders in obese patients presenting with acute respiratory failure. Respir Med 2005; 99:718-725

503. Buckle P, Pouliot Z, Millar T, et al: Polysomnography in acutely ill intensive care unit patients. Chest 1992; 102:288-291

504. Richards KC, O'Sullivan PS, Phillips RL: Measurement of sleep in critically ill patients. J Nurs Meas 2000; 8:131-144

505. Fontaine DK: Measurement of nocturnal sleep patterns in trauma patients. Heart Lung 1989; 18:402-410

506. Edwards GB, Schuring LM: Pilot study: Validating staff nurses' observations of sleep and wake states among critically ill patients, using polysomnography. Am J Crit Care 1993; 2:125-131

507. Kamdar BB, Shah PA, King LM, et al: Patient-nurse interrater reliability and agreement of the Richards-Campbell sleep questionnaire. Am J Crit Care 2012; 21:261-269

508. Bridoux A, Thille AW, Quentin S, et al: Sleep in ICU: Atypical sleep or atypical electroencephalography? Crit Care Med 2014; 42:e312-e313

509. Cho MY, Min ES, Hur MH, et al: Effects of aromatherapy on the anxiety, vital signs, and sleep quality of percutaneous coronary intervention patients in intensive care units. Evid Based Complement Alternat Med 2013; 2013:381381

510. Lytle J, Mwatha C, Davis KK: Effect of lavender aromatherapy on vital signs and perceived quality of sleep in the intermediate care unit: $\mathrm{A}$ pilot study. Am J Crit Care 2014; 23:24-29
511. Chen JH, Chao YH, Lu SF, et al: The effectiveness of valerian acupressure on the sleep of ICU patients: A randomized clinical trial. Int J Nurs Stud 2012; 49:913-920

512. Su CP, Lai HL, Chang ET, et al: A randomized controlled trial of the effects of listening to non-commercial music on quality of nocturnal sleep and relaxation indices in patients in medical intensive care unit. J Adv Nurs 2013; 69:1377-1389

513. Le Guen M, Nicolas-Robin A, Lebard C, et al: Earplugs and eye masks vs routine care prevent sleep impairment in postanaesthesia care unit: A randomized study. Br J Anaesth 2014; 112:89-95

514. Jones C, Dawson D: Eye masks and earplugs improve patient's perception of sleep. Nurs Crit Care 2012; 17:247-254

515. Richardson A, Allsop M, Coghill E, et al: Earplugs and eye masks: Do they improve critical care patients' sleep? Nurs Crit Care 2007; 12:278-286

516. Bourne RS, Mills GH, Minelli C: Melatonin therapy to improve nocturnal sleep in critically ill patients: Encouraging results from a small randomised controlled trial. Crit Care 2008; 12:R52

517. Shilo L, Dagan Y, Smorjik Y, et al: Effect of melatonin on sleep quality of COPD intensive care patients: A pilot study. Chronobiol Int 2000; 17:71-76

518. Ibrahim MG, Bellomo R, Hart GK, et al: A double-blind placebocontrolled randomised pilot study of nocturnal melatonin in tracheostomised patients. Crit Care Resusc 2006; 8:187-191

519. Erland LA, Saxena PK: Melatonin natural health products and supplements: Presence of serotonin and significant variability of melatonin content. J Clin Sleep Med 2017; 13:275-281

520. Hatta K, Kishi Y, Wada K, et al; DELIRIA-J Group: Preventive effects of ramelteon on delirium: A randomized placebo-controlled trial. JAMA Psychiatry 2014; 71:397-403

521. Nishikimi M, Numaguchi A, Takahashi K, et al: Effect of administration of ramelteon, a melatonin receptor agonist, on duration of stay in the ICU: A single-center, randomized, placebo-controlled trial. Crit Care Med 2018; 46:1099-1105

522. Wu XH, Cui F, Zhang C, et al: Low-dose dexmedetomidine improves sleep quality pattern in elderly patients after noncardiac surgery in the intensive care unit: A pilot randomized controlled trial. Anesthesiology 2016; 125:979-991

523. Oto J, Yamamoto K, Koike S, et al: Sleep quality of mechanically ventilated patients sedated with dexmedetomidine. Intensive Care Med 2012; 38:1982-1989

524. Treggiari-Venzi M, Borgeat A, Fuchs-Buder $T$, et al: Overnight sedation with midazolam or propofol in the ICU: Effects on sleep quality, anxiety and depression. Intensive Care Med 1996; 22:1186-1190

525. Engelmann C, Wallenborn J, Olthoff D, et al: Propofol versus flunitrazepam for inducing and maintaining sleep in postoperative ICU patients. Indian J Crit Care Med 2014; 18:212-219

526. Burgers JS, Grol R, Klazinga NS, et al; AGREE Collaboration: Towards evidence-based clinical practice: An international survey of 18 clinical guideline programs. Int J Qual Health Care 2003; 15:31-45

527. Hu RF, Jiang XY, Zeng YM, et al: Effects of earplugs and eye masks on nocturnal sleep, melatonin and cortisol in a simulated intensive care unit environment. Crit Care 2010; 14:R66

528. Li SY, Wang TJ, Vivienne Wu SF, et al: Efficacy of controlling nighttime noise and activities to improve patients' sleep quality in a surgical intensive care unit. J Clin Nurs 2011; 20:396-407

529. Patel J, Baldwin J, Bunting P, et al: The effect of a multicomponent multidisciplinary bundle of interventions on sleep and delirium in medical and surgical intensive care patients. Anaesthesia 2014; 69:540-549

530. Ahn HS, Kim HJ: Development and implementation of clinical practice guidelines: Current status in Korea. J Korean Med Sci 2012; 27(Suppl):S55-S60

531. Grol R: Successes and failures in the implementation of evidence-based guidelines for clinical practice. Med Care 2001; 39:I146-1154 
532. Woolf SH, Grol R, Hutchinson A, et al: Clinical guidelines: Potential benefits, limitations, and harms of clinical guidelines. BMJ 1999; 318:527-530

533. Nuckols TK, Lim YW, Wynn BO, et al: Rigorous development does not ensure that guidelines are acceptable to a panel of knowledgeable providers. $J$ Gen Intern Med 2008; 23:37-44

534. Mehta S, Burns KEA, Machado FR, et al: Gender parity in Critical Care Medicine. Am J Respir Crit Care Med 2017; 196:425-429

535. Deans KJ, Minneci PC, Danner RL, et al: Practice misalignments in randomized controlled trials: Identification, impact, and potential solutions. Anesth Analg 2010; 111:444-450
536. Muscedere J, Waters B, Varambally A, et al: The impact of frailty on intensive care unit outcomes: A systematic review and meta-analysis. Intensive Care Med 2017; 43:1105-1122

537. Scotts A: Minimizing mistakes and embracing uncertainty. PLoS Med 2015; 2:e272

538. Shekelle P, Woolf S, Grimshaw JM, et al: Developing clinical practice guidelines: Reviewing, reporting, and publishing guidelines; updating guidelines; and the emerging issues of enhancing guideline implementability and accounting for comorbid conditions in guideline development. Implement Sci 2012; 7:62 